Review

\title{
Phenotyping of Drosophila Melanogaster-A Nutritional Perspective
}

\author{
Virginia Eickelberg *, Kai Lüersen, Stefanie Staats and Gerald Rimbach
}

Citation: Eickelberg, V.; Lüersen, K.; Staats, S.; Rimbach, G. Phenotyping of Drosophila Melanogaster-A Nutritional Perspective. Biomolecules 2022, 12, 221. https://doi.org/ 10.3390/biom12020221

Academic Editor: Anika Wagner

Received: 20 December 2021

Accepted: 20 January 2022

Published: 27 January 2022

Publisher's Note: MDPI stays neutral with regard to jurisdictional claims in published maps and institutional affiliations.

Copyright: (C) 2022 by the authors. Licensee MDPI, Basel, Switzerland. This article is an open access article distributed under the terms and conditions of the Creative Commons Attribution (CC BY) license (https:// creativecommons.org/licenses/by/ $4.0 /)$.
Department of Food Science, Institute of Human Nutrition and Food Science, University of Kiel, Hermann-Rodewald-Strasse 6-8, D-24118 Kiel, Germany; luersen@foodsci.uni-kiel.de (K.L.); staats@foodsci.uni-kiel.de (S.S.); rimbach@foodsci.uni-kiel.de (G.R.)

* Correspondence: eickelberg@foodsci.uni-kiel.de; Tel.: +49-431-880-5313

\begin{abstract}
The model organism Drosophila melanogaster was increasingly applied in nutrition research in recent years. A range of methods are available for the phenotyping of D. melanogaster, which are outlined in the first part of this review. The methods include determinations of body weight, body composition, food intake, lifespan, locomotor activity, reproductive capacity and stress tolerance. In the second part, the practical application of the phenotyping of flies is demonstrated via a discussion of obese phenotypes in response to high-sugar diet (HSD) and high-fat diet (HFD) feeding. HSD feeding and HFD feeding are dietary interventions that lead to an increase in fat storage and affect carbohydrate-insulin homeostasis, lifespan, locomotor activity, reproductive capacity and stress tolerance. Furthermore, studies regarding the impacts of HSD and HFD on the transcriptome and metabolome of $D$. melanogaster are important for relating phenotypic changes to underlying molecular mechanisms. Overall, D. melanogaster was demonstrated to be a valuable model organism with which to examine the pathogeneses and underlying molecular mechanisms of common chronic metabolic diseases in a nutritional context.
\end{abstract}

Keywords: Drosophila melanogaster; high-sugar diet; high-fat diet; obesity; phenotyping

\section{Introduction}

Since the early 20th century, the fruit fly, Drosophila melanogaster, has been used as an important model organism in experimental research. The use of $D$. melanogaster provides several advantages. First, the number of offspring per fertilized female is relatively large, while the life cycle is short. The developmental time from egg to hatched fly is, on average, nine to ten days at $25^{\circ} \mathrm{C}$ [1]. This ensures the availability of a sufficient number of fruit flies for research in a short period of time. Second, the cultivation and maintenance of D. melanogaster in large numbers are practicable, and the costs are moderate. Furthermore, D. melanogaster has a comparatively short lifespan, with a maximum lifespan of approximately 60 days for males and 80 days for females depending on the holding conditions and strains, which allows lifelong examinations. Further advantages are that the genome of D. melanogaster is completely sequenced [2] and that nearly $75 \%$ of the disease-related genes in humans have functional orthologous genes in D. melanogaster [3].

Common fields of application for D. melanogaster as a model organism are genetics, developmental biology and biomedicine. Specific mutants and transgenic flies can be used as models that mimic various chronic diseases [4-7].

In recent years, the value of $D$. melanogaster in nutrition research became increasingly recognized. In this context, the roles of different dietary compounds, diet compositions and plant bioactive components on various readouts were investigated [8-12]. As a standard diet, a complex medium comprising yeast, sugar and agar is commonly used, which can be easily and rapidly prepared, providing all of the necessary nutrients to flies [4]. However, to unravel the influence of distinct dietary factors, semidefined or fully defined diets, such as holidic media, represent helpful alternatives [13]. 
A variety of methods can be applied for the phenotyping of D. melanogaster. Important readouts in nutritional studies are measurements of food intake, body weight and composition, as well as metabolic and digestive function. Other important phenotypes are lifespan, fitness, locomotor activity, responses to environmental stresses and reproductive and developmental capacity $[4,14]$.

In the first part of this review, methods for phenotyping $D$. melanogaster in a nutritional context are outlined, and the advantages and disadvantages of the different methods are illustrated. In the second part, the application of comprehensive phenotyping is demonstrated with examples of dietary interventions, namely high-sugar diet (HSD) and high-fat diet (HFD) feeding. Finally, studies regarding the impacts of HSD and HFD feeding on the transcriptome and metabolome of $D$. melanogaster are presented, which place the nutritional phenotypes in the context of underlying cellular and molecular mechanisms.

\section{Phenotyping of D. melanogaster}

\subsection{Body Weight}

An important step in nutrition research in flies was made by Mackay and coworkers through the quantification of genetic variation in body weight and major metabolites (such as glycogen, triacylglyceride (TAG)) and their association with obesity and the metabolic rate in flies [15]. For the determination of body weight, flies have to be sex-specifically pooled after prefeeding. Following transfer to sealable vials, groups of at least 10-20 animals are weighed with a precision scale, and the average body weight per fly is calculated [14]. Additionally, the dry mass of D. melanogaster can be quantified. To this end, the fruit flies are dried in an oven (e.g., at $60^{\circ} \mathrm{C}$ for $24 \mathrm{~h}$ ) before their body mass is determined with a precision scale [16]. By calculating the difference between wet and dry weight, the water content of the flies can be assessed.

\subsection{Body Composition: Lipids, Proteins, Carbohydrates}

\subsubsection{Lipid Determination}

An overview of the different methods for the determination of fat content, with advantages and disadvantages, is provided in Table 1 . Body fat in D. melanogaster consists mainly of TAGs, which are stored in lipid droplets of adipocytes [17]. The number and size of lipid droplets differ depending on the type of tissue and environmental conditions, such as the ingested diet. Lipid droplets can be evaluated and quantified visually by staining with dyes such as Nile red, BODIPY or Harris hematoxylin [18-20]. This method is applicable for examination of tissues at the cellular level but is limited in the quantification of the total TAG content of adult flies due to the different fat-accumulating organs, such as the fat body, intestine and ovaries [17].

To determine whole-body TAG content, a fly homogenate is initially made via grinding of the flies in a buffer solution and subsequent centrifugation (Figure 1). Commonly applied methods for TAG measurement are coupled colorimetric assays (CCAs), for which kits from various manufacturers are available. CCAs are generally based on the same reaction scheme, which starts with the enzymatic hydrolysis of TAGs by lipoprotein lipase into glycerol and free fatty acids (FA) [17,21-24]. The glycerol is then enzymatically phosphorylated and oxidized, generating hydrogen peroxide that forms a red quinoneimine dye with 4-aminoantipyrine and 3,5-dichloro-2-hydroxybenzene sulfonate in the last reaction step. The absorption of this red dye is proportional to the TAG concentration of the sample $[25,26]$. These CCAs enable the rapid determination of the TAG content with a large sample size. However, owing to the insolubility of the stored fats in adipocytes of D. melanogaster, the accuracy of the determination of absolute TAG levels with CCAs is questioned. Additionally, the identification of the proportions of different glycerides in the initial sample is not possible because of the determination of the total glycerol content at the final reaction step. However, the free glycerol present in flies is reported to be neglectable [17]. It should also be noted that the colored eye pigments of D. melanogaster can distort the results, so removal of the heads prior to measurement is recommended [27]. 
Table 1. Methodological overview of the determination of triacylglycerides (TAGs) with advantages and disadvantages.

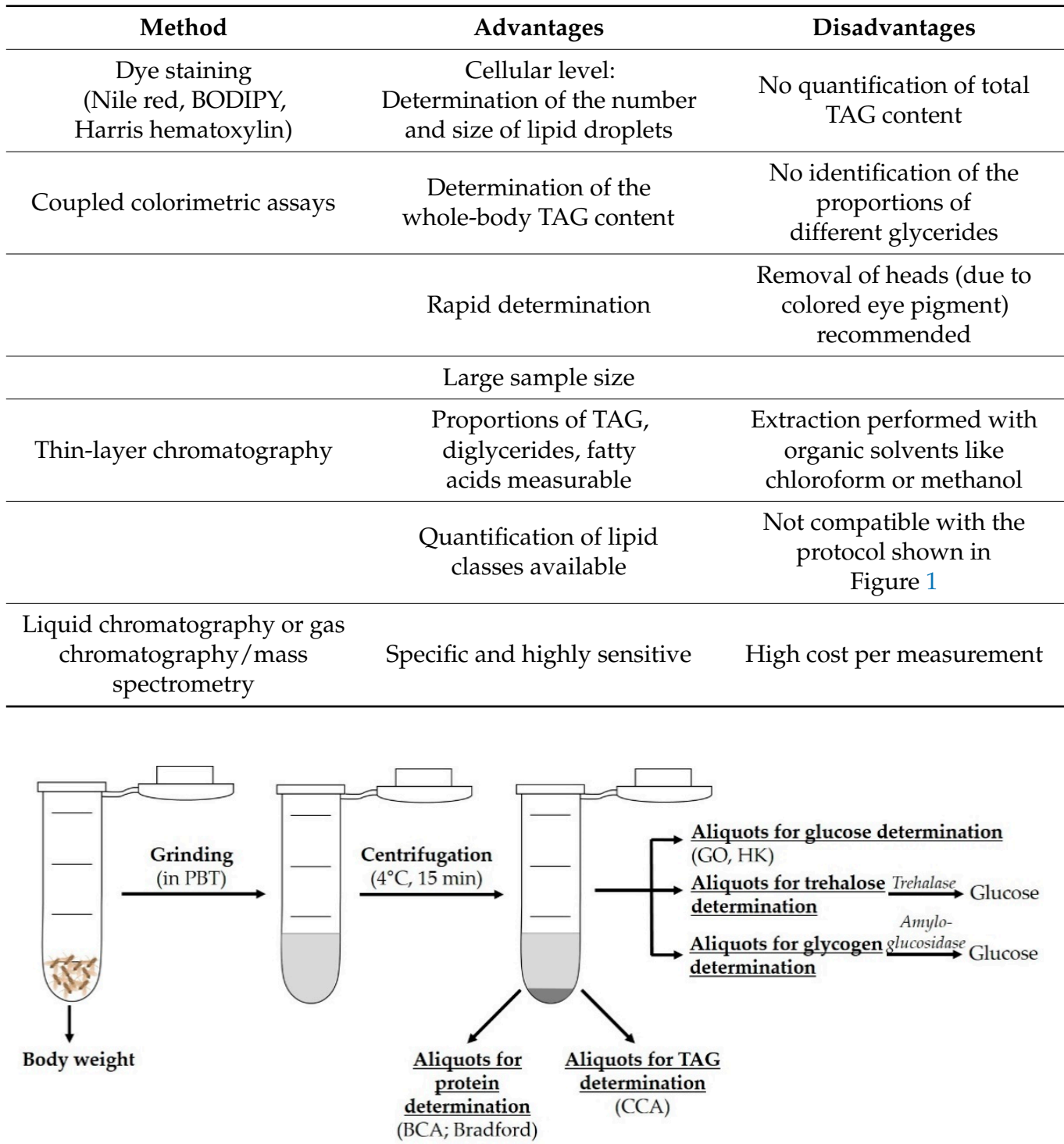

Figure 1. Exemplary protocol for assessment of the body composition of Drosophila melanogaster. After determining their body weight, flies are ground in Dulbecco's phosphate-buffered saline with Triton X-100 (PBT) by using a tissue homogenizer. Following centrifugation, aliquots of the supernatant are utilized for further measurements. The protein content is determined with a bicinchoninic acid (BCA) or Bradford assay, the TAG content with coupled colorimetric assays (CCAs) and the glucose content with enzymatic methods based on the enzyme glucose oxidase (GO) or hexokinase (HK). For assessment of trehalose and glycogen concentrations, enzymatic treatments with trehalase and amyloglucosidase are conducted, respectively, after which free glucose is determined (modified from [28]).

Another method for lipid quantification in D. melanogaster is thin-layer chromatography (TLC, Figure 2). The extraction of lipids with a mixture of two or three organic solvents, such as chloroform or methanol, in different mass ratios is necessary for TLC. The extraction techniques of Bligh and Dyer [29] or Folch [30] are appropriate for D. melanogaster. The extracted lipid samples and lipid standards are separated on a plastic or glass TLC plate coated with silica (stationary phase), and the liquid (mobile) phase is hexane, ethyl ether and acetic acid. The TLC plate is then air-dried and stained with, e.g., copper (II) sulfate pentahydrate or ceric ammonium molybdate solution depending on the fat group 
for detection. With TLC, the proportions of TAGs, diglycerides and FAs in the sample can be measured. In addition, quantification of the lipid classes by the application of photodensitometry is possible [17,31,32].

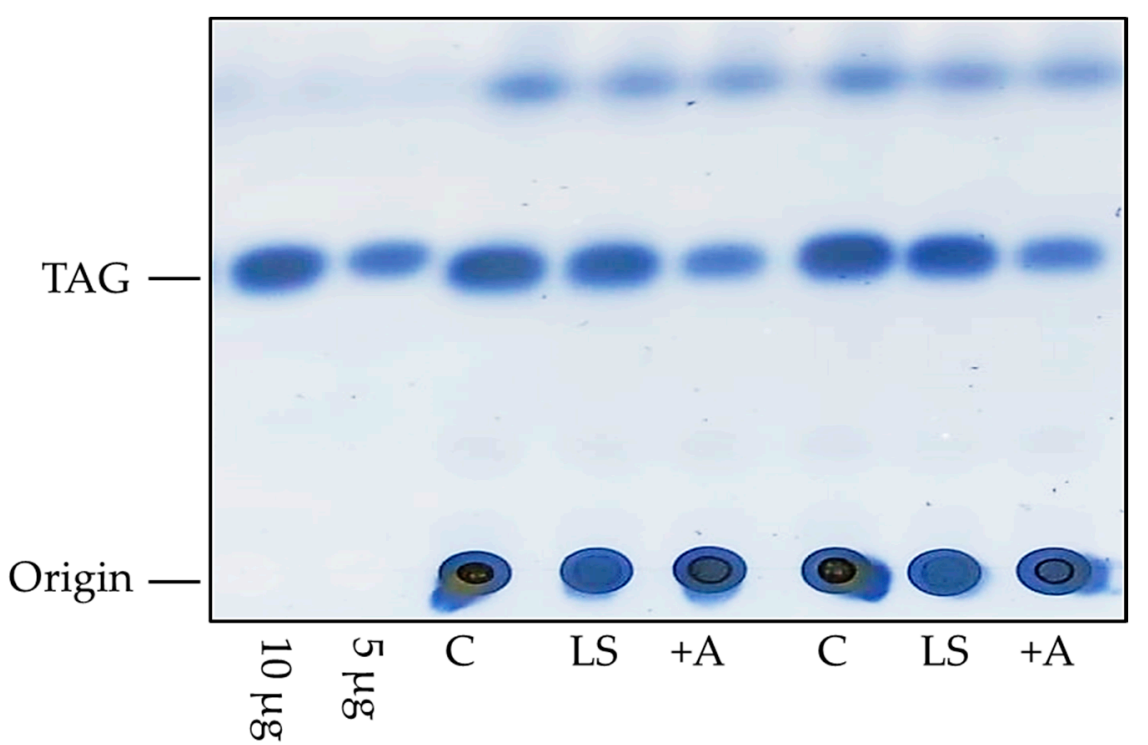

Figure 2. Example of triacylglyceride (TAG) determination in Drosophila melanogaster by thin-layer chromatography (TLC). Starting with eggs, fruit flies were raised on different experimental diets, and females were harvested 10 days after eclosion. TAGs were extracted from the flies, separated by TLC and stained according to the methods of Al-Anzi et al. (2009) [31]. A low-starch diet (LS) and acarbose treatment $(+\mathrm{A})$ led to decreased TAG levels. C, control diet containing $10 \%$ starch; LS, low-sugar diet containing $1 \%$ starch; $+\mathrm{A}$, control diet supplemented with $1.8 \mathrm{mg} / \mathrm{L}$ acarbose. Lard was used as a standard (the two bands on the left).

For the determination and quantification of different lipid types, a more specific and highly sensitive analytical method is needed. For this purpose, gas chromatography/mass spectrometry (GC/MS) or liquid chromatography/mass spectrometry (LC/MS) are suitable. After extraction (e.g., according to the methods of Bligh and Dyer [29] or Folch [30]), samples are dissolved in a mobile phase such as ammonium acetate in methanol/chloroform or methanol/water and injected onto a silica column [33-35]. Although a precise analysis of lipids is possible with GC/MS or LC/MS, a disadvantage lies in the relatively high cost per measurement.

\subsubsection{Protein Determination}

Whole-body protein content of $D$. melanogaster is usually determined by assays based on colorimetric or fluorescent signals. The supplies can be obtained as test kits from various commercial suppliers. In these assays, the protein content is measured in the supernatant of a sample after homogenization and centrifugation (Figure 1). The two most common methods for protein determination are the Bradford assay and the bicinchoninic acid (BCA) assay. Both are colorimetric assays, feasible in test-tube or microplate format, using bovine serum albumin (BSA) as a standard protein [36-38].

The Bradford assay can be applied rapidly and simply at room temperature with only a minimal and tolerable influence of substances such as thiols, metal chelators or reducing agents. However, one must keep in mind that the measurement might be affected by surfactants such as Triton X-100 or a strong alkaline buffer, which can cause precipitation. Thus, the concentrations of these substances must be considered and adjusted if necessary [36,37].

The BCA assay is also rapid and simple to perform. Fewer protein-protein variations occur in the BCA assay than in the Bradford assay. Additionally, many surfactants can be applied without adverse effects on the BCA assay outcome. However, a multiplicity of 
substances in solvents or buffers (e.g., ascorbic acid, iron) have copper-reducing or chelating capabilities, which could influence the test results. The removal of these interfering substances is mandatory for precise protein determination [37,38].

\subsubsection{Carbohydrate Determination}

For the phenotyping of D. melanogaster, the determination of glucose, trehalose and glycogen could also be of importance. Depending on the research objectives and parameters, such as the developmental stage of the fly, free glucose can be measured in whole-body homogenates or in hemolymph. For the determination of glucose in wholebody homogenates, flies are initially ground in PBT and centrifuged, and the supernatant is subsequently used for a glucose assay (Figure 1). The measurement of the whole-body homogenate can be rapidly conducted, and the sample throughput is high. However, the intestinal glucose content, derived from the diet, could affect the determination. Additionally, the differentiation between circulating and stored glucose concentrations cannot be made due to the continuous exchange between intracellular and extracellular components; thus, only an approximate content of free glucose can be obtained [39].

A precise measurement of free circulating glucose is possible via the quantification of glucose in the hemolymph of the fly, which is collected from the head or thorax by capillary action or puncture with subsequent centrifugation [40].

Two common enzymatic methods are available to measure free glucose in the hemolymph or in whole-body homogenate. In the first method, the enzyme glucose oxidase (GO) is used to catalyze the oxidation of glucose via the production of gluconic acid and hydrogen peroxide. Subsequently, hydrogen peroxide reacts with various chromophores in combination with peroxidase to produce a colored product that is photometrically or fluorometrically assessed, enabling the calculation of the glucose concentration [41,42].

In the second method, D-glucose is converted by two consecutive enzymatic steps into gluconate-6-phosphate, resulting in the formation of reduced nicotinamide-adenine dinucleotide phosphate (NADPH), which is measured spectrophotometrically. The amount of NADPH synthesized is stoichiometrically related to the glucose concentration of the sample [39,42].

For measurement of trehalose, the disaccharide is initially split by the enzyme trehalase into two D-glucose monomers, which can be assessed enzymatically as described above.

Glycogen in adult flies is primarily located in the fat body, muscle tissue and intestine and can be visualized by periodic acid-Schiff staining or quantified by enzymatic methods. For this, the sample is incubated with the enzyme amyloglucosidase, which cleaves glycogen into free glucose, which then can be measured as described above [28].

\subsection{Metabolic Rate}

The metabolic rate reveals information about health and fitness status and is influenced by factors such as environmental temperature [43], group interactions [44], age, physical activity and diet [45]. In recent publications, $\mathrm{CO}_{2}$ measurements via respirometry were introduced in Drosophila research, leading primarily to the identification of sensory perception, which induced aversive cues and modulates lifespan through serotonergic signaling [46]. The metabolic rate of D. melanogaster is assessed by either direct or indirect calorimetric measurements.

For direct calorimetric measurement, the flies are placed in a calorimetric experimental chamber with a sensitive thermostat, where the heat produced by the organism is determined. The metabolic rates of different flies can be recorded in parallel in real time. To increase the informative value, the measurement can be completed by recording the fly's movements [45]. The direct calorimetric determination of metabolic rate enables the assessment of aerobic and anaerobic catabolism [47]. A disadvantage of this direct technique is the requirement of specific, mostly custom-built equipment.

During indirect calorimetric determination (respirometry), flies are placed in a sealed experimental chamber with flow-through and optional flushing with $\mathrm{CO}_{2}$-free room air. 
The changes in the gas concentration in the chamber are measured with a gas analyzer, and the oxygen $\left(\mathrm{O}_{2}\right)$ consumption and/or $\mathrm{CO}_{2}$ production per time interval is inferred. The metabolic rate of $D$. melanogaster is calculated based on the respiratory quotient as the ratio of produced $\mathrm{CO}_{2}$ to consumed $\mathrm{O}_{2}[48,49]$.

As an alternative to complex measuring instruments, the amount of produced $\mathrm{CO}_{2}$ can be determined using a self-assembled respirometer, which is built from a pipette tip, micropipette and $\mathrm{CO}_{2}$-absorbing soda lime (Figure 3). The flies, which are inserted in the respirometer, convert $\mathrm{O}_{2}$ into $\mathrm{CO}_{2}$. Since $\mathrm{CO}_{2}$ is trapped by soda lime, the gas volume in the chamber will decrease over time. This can be monitored by the rising level of the dyed water in the connected micropipettes. By using image processing software, the distances of the capillary filling within a certain period of time are determined to calculate the amount of $\mathrm{CO}_{2}$ produced per fly and time. This method is simple and cost-effective, and the metabolic rate of flies from different treatments can be studied simultaneously [43]. However, the assumption in respirometry of approximate values for the correlation between gas exchange and metabolic substrate utilization, especially in smaller organisms, leads to a high error rate for the indirect caloric determination of metabolic rate compared to direct measurements. Additionally, the metabolic rate can be estimated by indirect calorimetry only for aerobic catabolism [47].

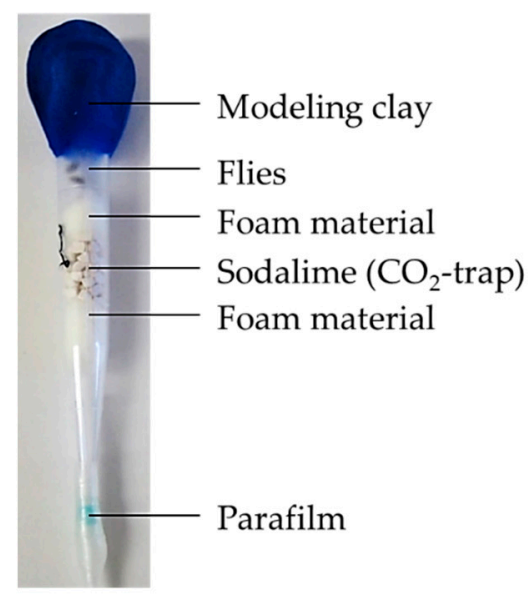

(a)

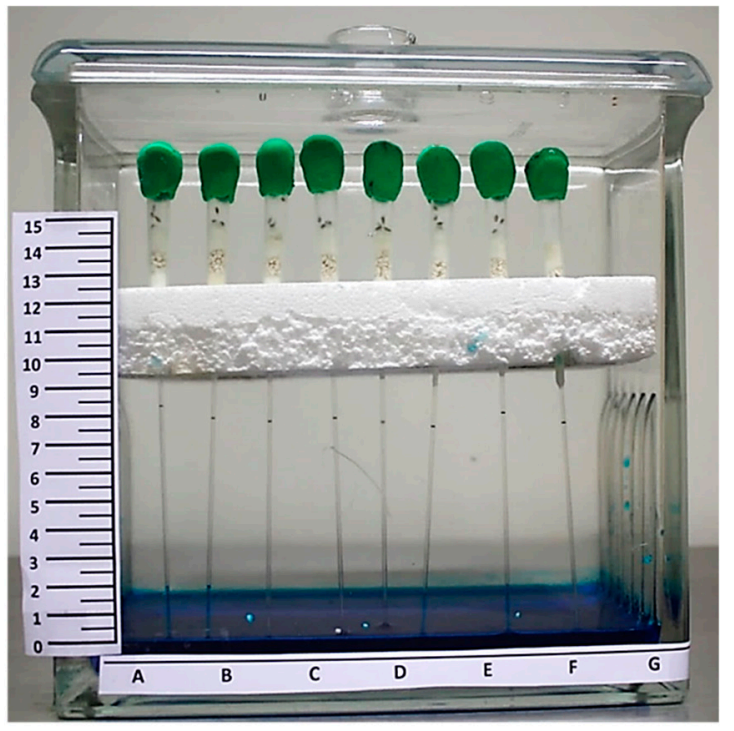

(b)

Figure 3. Schematic of the self-assembled respirometer setup. (a) The respirometer is a small metabolic chamber built from a pipette tip, a micropipette, Parafilm, foam and $\mathrm{CO}_{2}$-absorbing soda lime. After brief anesthetization, flies are placed into the respirometer chamber, which is subsequently closed with a plug of food and covered with plasticine. (b) After an adaptation period, the respirometers are inserted into a holder in an outer chamber so that the micropipette tips are immersed into a layer of dyed water. The lid of the outer chamber is closed, and a first photograph is taken. The changes in the filling volume of the micropipettes are documented with additional photographs. By using image processing software, the distances of capillary filling are determined. The amount of $\mathrm{CO}_{2}$ produced per fly and hour is calculated via the formula: (micropipette radius $\times$ ratio of capillary filling of the micropipette at the start and end point for the sample-negative control) $\times 1000) /($ number of flies $\times$ duration of exposure) (modified from [43]).

\subsection{Food Intake}

In the following section, several methods for the indirect determination of food intake are introduced. One option for food intake monitoring is the use of trackable supplements such as radioisotope-labeled metabolites or nonabsorbable dyes (Brilliant Blue FCF or Sulforhodamine B) (Figure 4) [10,50-53]. In the case of dyes, flies are fed on the labeled 
medium for a defined period of time. Subsequently, the amount of dye that has accumulated in the digestive tract, which reflects food intake, is quantified in fly lysates by using a spectrophotometer or fluorescence reader. In this method, it is important to consider the background pigmentation of the fly lysates. Although simple, this dye accumulation method is thought to have several pitfalls. Most importantly, the incubation time is critical since the linearity of accumulation ends when excretion of the supplement begins. Hence, intake rates can be determined solely during the short ( $<30 \mathrm{~min}$ ) initial phase of feeding, and long-term measurement of food intake becomes inaccurate [54]. However, short feeding periods generate substantial biological variability. Furthermore, flies have to be sacrificed, making it impossible to monitor the food intake of a particular fly population over time.

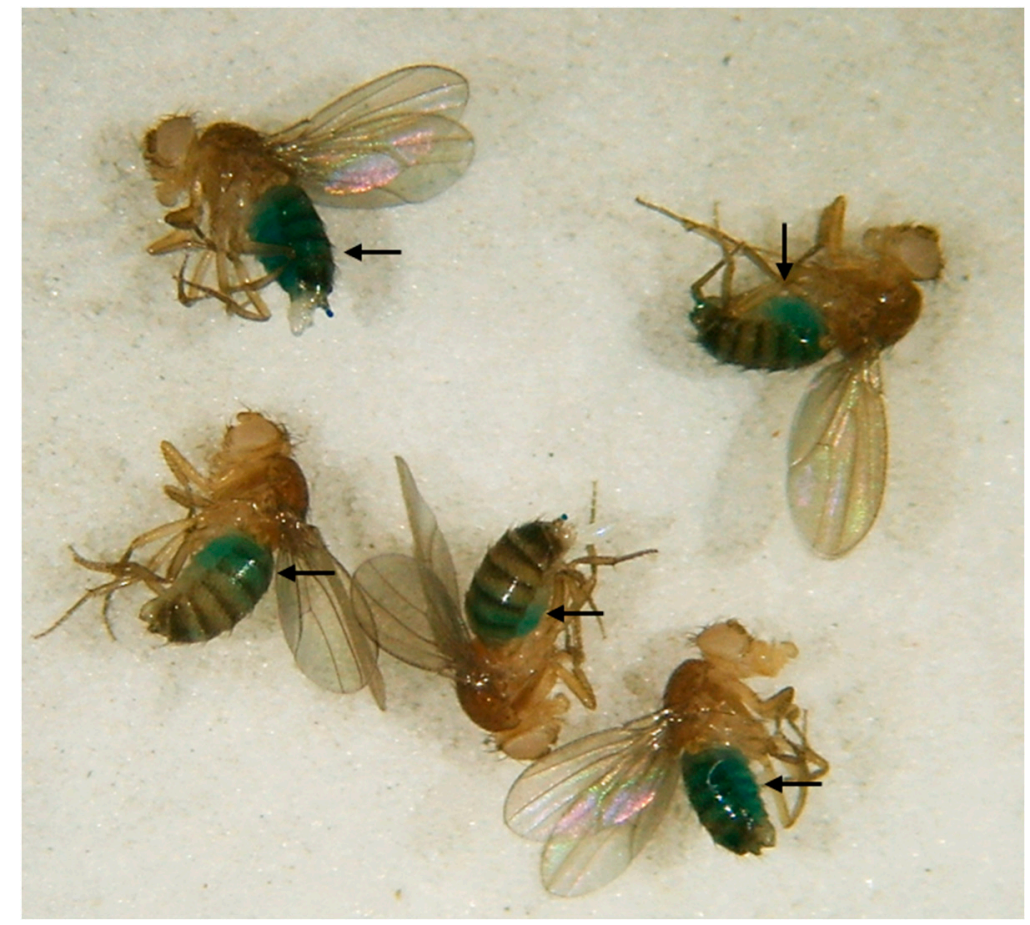

Figure 4. Determination of food intake with dyed medium. Flies are fed on medium supplemented with the nonabsorbable dye, Brilliant Blue FCF. After a defined period of time, the animals are removed, and dye accumulation within the digestive tract is indicated via assessment of the bluecolored abdomen (black arrows). The amount of ingested dye can be determined in the fly lysate by a spectrophotometer. The background absorption of fly lysates must be considered (modified from [10]).

To circumvent the limitations of dye accumulation assays, a consumption-excretion (Con-Ex) method was recently established, which enables the long-term measurement of food intake. Flies are transferred to an empty vial containing a colored medium in a small feeder cup in the lid. After a defined feeding period, the ingested dye content is determined by homogenization of the flies. Additionally, the excreted dye, which is spread on the walls of the vials, is collected, and the absorption of the collected dye fractions (fly lysate and dissolved excreta) is measured by a spectrophotometer. Food intake is calculated using a standard curve [55].

Excreta quantification (EX-Q), a modification of the Con-Ex method also enables the determination of food intake without fly sacrifice (Figure 5). A reduction in the surface of the feeder cap enables the quantification of the complete food intake using the excretions [56]. 


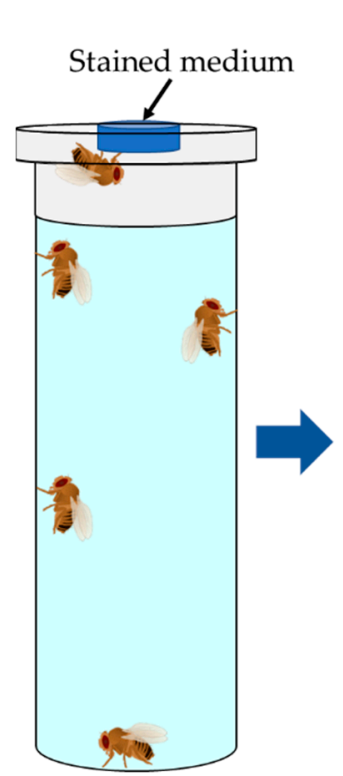

(a)

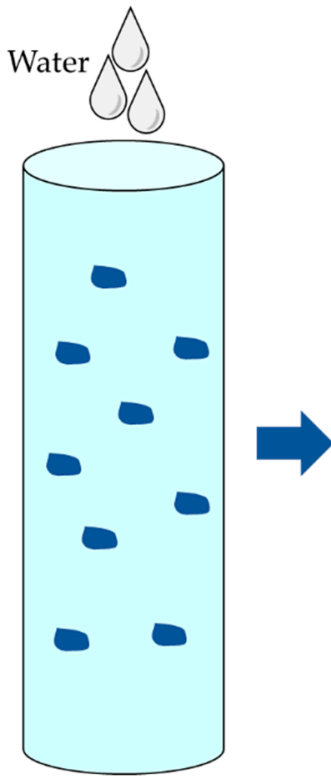

(b)

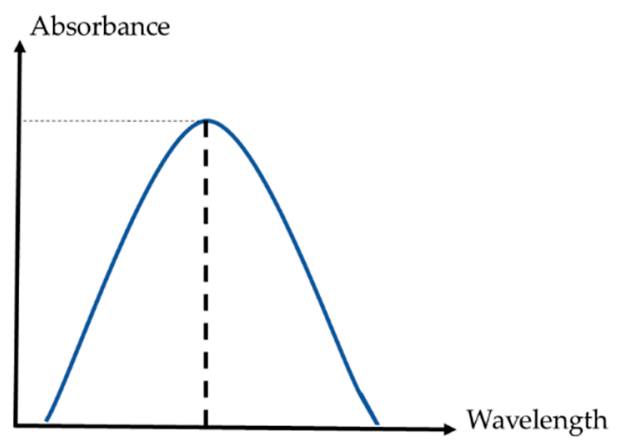

(c)

Figure 5. Excreta quantification (EX-Q). (a) For EX-Q, flies ingest a medium stained with a nonabsorbent dye in the overhead position, and the dye is excreted with the feces. (b) The flies are removed, and the vials with the fecal spots are rinsed with water. (c) The absorption is measured photometrically, and the amount of ingested food is quantified with a standard curve (modified from [56]).

As an alternative to dyes, the medium can be supplemented with radioactively labeled substances such as $\left[{ }^{14} \mathrm{C}\right]$-choline, $\left[{ }^{3} \mathrm{H}\right]$-thymidine or [ $\left.\alpha-32 \mathrm{P}\right]-\mathrm{dCTP}[57-63]$. Similar to the methods using dyed medium, flies are fed on labeled medium for a certain period of time. In most cases, the ingested food volume is quantified with liquid scintillation counting. However, radioisotopes such as $[\alpha-32 \mathrm{P}]$ can be measured through the cuticle so that the homogenization of flies is not necessary. This method is described as very reliable and sensitive. However, radioactive-labeled metabolites differ in their adsorption rates in the digestive tract, which can lead to retention of radioisotopes, leading to the underestimation of food intake [64].

The direct measurement of food intake can be obtained by the CApillary FEeder (CAFE) assay (Figure 6) $[65,66]$. In this assay, flies are kept in vials under constant humidity. Capillaries containing the liquid medium are placed vertically through the lids of the vials. Flies absorb the liquid by feeding on the ends of the capillaries. Consequently, by measuring the drop-in volume in the capillary, the amount of food intake can be determined. This assay can be used for quantification of ingested food, as well as for choice experiments. An advantage of the CAFE assay is the direct measurement of food intake without the need to anesthetize or kill flies, which enables the long-term monitoring of food intake under different conditions (e.g., lifespan experiments). The CAFE assay is described as an accurate and consistent method [64], whereas it is not suitable for all fly strains in view of the overhead position of the food source, since flies with mobility impairments are unable to reach the feeding place. In addition, only liquid media, and not the commonly used agar-based medium, can be applied in this assay [56]. Garlapow and coworkers performed genome-wide association studies for food intake and environmental variance of food intake, which resulted in the identification of numerous novel genes, related to feeding behavior [67]. 


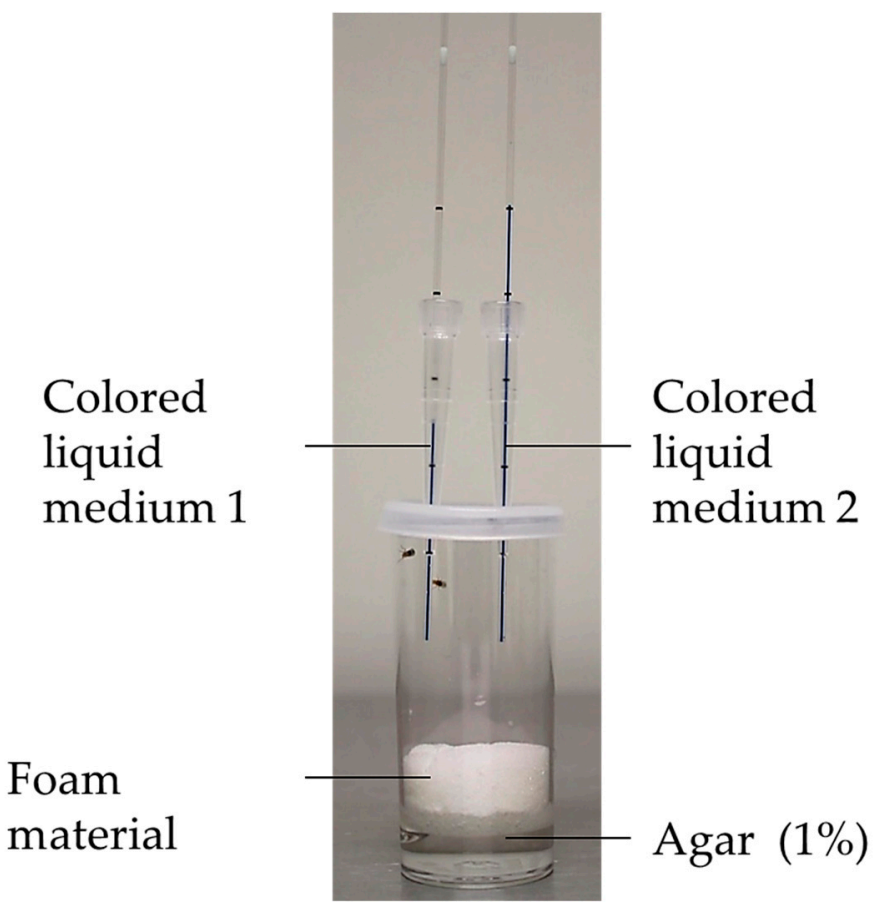

Figure 6. CApillary FEeder (CAFE) assay. The CAFE assay can be used for quantification of food intake and for food choice experiments. Flies are transferred into a vial, which is closed with a lid with small air holes. Capillaries filled with liquid medium and placed into pipette tips are inserted vertically through these air holes. Subsequently, the vials are kept in a storage box to maintain a constant humidity level. The flies absorb the liquid food by feeding on the lower ends of the capillaries. As time passes, the fill volumes of the capillaries decrease. The amount of ingested food can be calculated by measuring the fill levels of each capillary at the start and end of the CAFE assay (modified from $[65,66]$ ).

For the MAnual FEeding (MAFE) assay, individual flies are carefully aspirated into a pipette tip following a starvation phase. A capillary with a liquid medium is placed on the proboscis of the fly, and the complete extension of the proboscis is monitored optically until there is no additional reaction to the feeding stimulus. The ingested volume and the duration of the food intake are recorded. The food intake of the individual fly can be recorded on a scale of seconds [68]. The MAFE assay should be performed by the same person each time, as the results strongly depend on the handling by the experimenter (e.g., position of the fly in the pipette tip). Additionally, this assay only works in starved flies and requires a high expenditure of time per application, which means that this assay only suitable for a small number of test species [68,69].

The fly Proboscis and Activity Detector (flyPAD) is an automated tool used to sensitively analyze eating behavior and food choice experiments with very small amounts of food. In this method, flies are placed, after a short starvation period, in a behavioral arena with two independent channels containing a solid medium. By contacting the food surface with the proboscis, a change in capacity is induced, which results in an electronic signal. The simultaneous recording of multiple behavioral arenas is possible; however, it is not suitable for large-scale experiments. Furthermore, the long-term recording of food intake is not practicable with the flyPAD [70].

The Fly Liquid-Food Interaction Counter (FLIC) is an automatic tool used to record food volume and choice via the continuous recording of the number and duration of each interaction of the fly (or group of flies) with a liquid medium. This allows for the recording of data on movement and the circadian feeding behavior of flies for short- and long-term periods [71]. The system can be extended with further phenotypic elements such as shock triggering, which enables the examination of food-related learning processes. 
The advantage of automated techniques lies in the standardization and independence of the experimenter; however, they are relatively cost-intensive.

The introduction of different methods for the determination of food intake shows the diversity of the methods. Each method offers merits and drawbacks and should be chosen according to the research purpose (Table 2). To increase the reliability of the results, it is useful to validate the results with an additional method.

\subsection{Lifespan}

For lifespan experiments, D. melanogaster cultures need to be initially synchronized (Figure 7). To this end, freshly laid eggs are collected from the plate using a buffer or a saline solution, rinsed several times and transferred to cultivation medium until hatching. A total of 100 age-matched flies per sex and condition are allocated to an appropriate number of experimental vials, and their survival is monitored and documented over time until the death of the last fly [72,73]. The flies are transferred to a vial with fresh medium every two to three days.

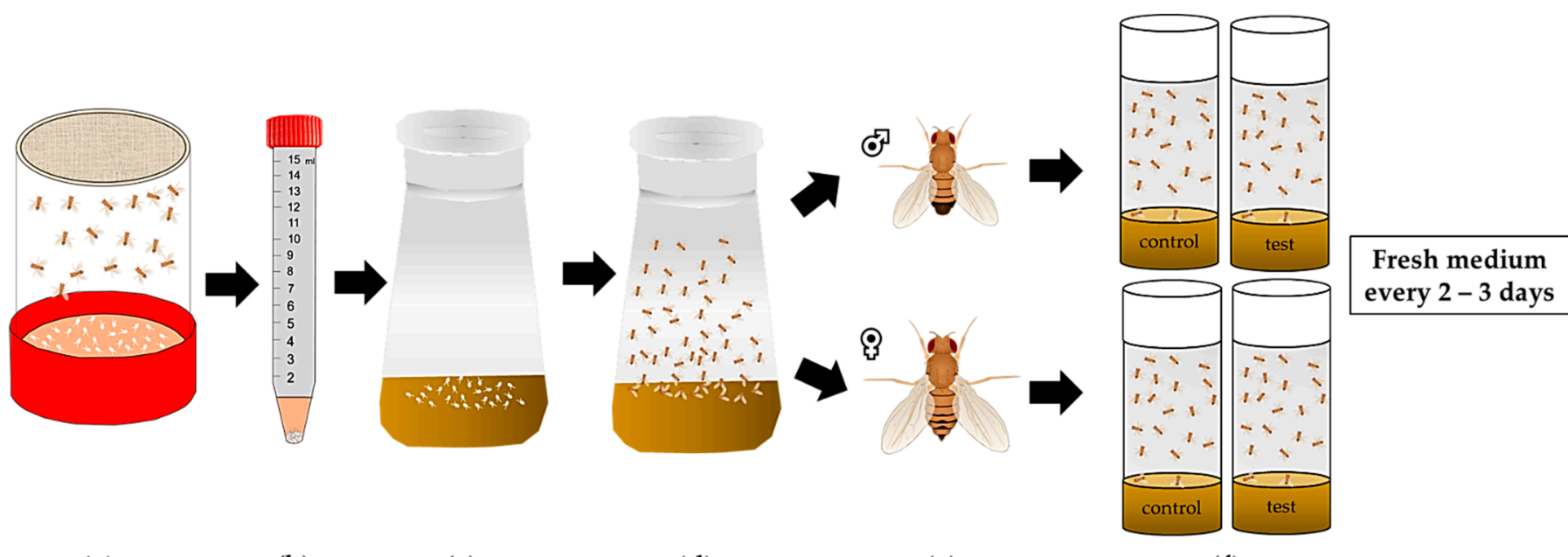

(a)

(b) (c)

(d) (e) (f)

Figure 7. Implementation of a lifespan experiment. (a) Adult flies are transferred into an egg-laying cage covered by a grape agar plate with active yeast paste. After $24 \mathrm{~h}$, the grape agar plate is replaced and inoculated with only a small spot of active yeast paste. (b) The next day, flies are removed from the cage, and the laid eggs are removed from the surface of the plate with a cotton swab and a physiological buffer or saline solution. The egg suspension is rinsed three times with buffer or saline solution before (c) aliquots of the egg suspension are transferred to cultivation medium, where egg-to-adult fly development occurs within approximately 10 days. (d) After hatching, the mixed fly populations are transferred to fresh standard medium for mating. (e) Forty-eight hours later, the adult flies are usually separated into male and female cohorts. (f) Approximately 20 to 30 flies per sex are placed in a vial with a control or experimental medium. Four to five replicates are used to obtain a total number of at least 100 flies per sex and treatment. The flies are transferred to fresh medium every 2 to 3 days, and the number of dead flies is documented at each transfer. This documentation is continued until all flies are dead (modified from [72,73]). 
Table 2. Advantages and disadvantages of methods used for the determination of food intake and food choice in Drosophila melanogaster.

\begin{tabular}{|c|c|c|}
\hline Method & Advantages & Disadvantages \\
\hline Dye labeling of the food & $\begin{array}{ll}- & \text { Solid medium } \\
-\quad & \text { Large fly groups }\end{array}$ & $\begin{array}{ll}\text { - } & \text { Indirect determination of food intake via marker } \\
\text { - } & \text { Inaccurate results (fast dye accumulation) } \\
\text { - } & \text { Flies must be killed for measurement }\end{array}$ \\
\hline $\begin{array}{l}\text { Consumption-excretion } \\
\text { (Con-Ex); } \\
\text { Excreta quantification } \\
\text { (EX-Q) }\end{array}$ & $\begin{array}{l}\text { - } \quad \text { Long-term measurement of food intake } \\
\text { No killing of flies (EX-Q) }\end{array}$ & $\begin{array}{l}\text { - Overhead or horizontal position of food source (not suitable for all } \\
\text { fly strains) }\end{array}$ \\
\hline $\begin{array}{l}\text { CApillary FEeder } \\
\text { (CAFE) assay }\end{array}$ & $\begin{array}{ll}\text { - } & \text { Applicable for food intake and food choice } \\
\text { - } & \text { Direct measurement of food intake } \\
\text { - } & \text { Accurate and consistent }\end{array}$ & $\begin{array}{l}-\quad \text { Overhead position of food source (not suitable for all fly strains) } \\
\text { - Only liquid medium }\end{array}$ \\
\hline Proboscis extension & $\begin{array}{ll}- & \text { Simple setup } \\
\text { - } & \text { Recording of individual flies } \\
& \text { High time resolution }\end{array}$ & $\begin{array}{l}\text { - } \quad \text { Highly dependent on experimenter } \\
\text { - } \quad \text { Limited time of application } \\
\text { Not suitable for larger fly groups and longer time periods }\end{array}$ \\
\hline $\begin{array}{l}\text { MAnual FEeding } \\
\text { (MAFE) assay }\end{array}$ & - No interference of foraging and feeding start & - $\quad$ Liquid medium in a capillary \\
\hline Automated detection of food intake & $\begin{array}{ll}\text { - } & \text { Sensitive } \\
\text { - } & \text { High resolution } \\
\text { - } & \text { Recording of individual flies } \\
\text { - } & \text { Simultaneous recording of feeding behavior of numerous flies } \\
\text { - } & \text { Recording independent of experimenter }\end{array}$ & $\begin{array}{l}\text { - No standard conditions of maintenance } \\
\text { - } \quad \text { Not suitable for larger fly groups } \\
\text { - } \quad \text { Expensive in comparison to other methods }\end{array}$ \\
\hline
\end{tabular}


Table 2. Cont.

- $\quad$ Short- and long-term observation

- Recording of movement pathways, food assessment, circadian

feeding behavior

- $\quad$ Linkage with other hardware, e.g., shock-triggering component

fly Proboscis and Activity

Detector(flyPAD)
- $\quad$ Solid medium
- Only liquid medium

- No long-term observation 
To investigate the long-term impact of different nutritional factors on the survival of fruit flies, lifespan experiments can be conducted. An approach to combining genetic traits with longevity and ageing was undertaken by the Pletcher lab $[74,75]$ through the examination of environmentally induced heterogeneity and its influence on age-specific genetic variance for mortality rates. Furthermore, the group applied genome-wide transcript profiling in various longevity and aging models, e.g., in calorically restricted Drosophila flies [76]. It was repeatedly documented that calorie restriction extends lifespan in various model organisms [77]. In this context, Rogina and Helfand reported that the transcription factor Sir2 is centrally involved in the lifespan-extending molecular pathway in Drosophila. An increase in Drosophila Sir2 prolonged lifespan, whereas a decrease in dSir2 inhibited the lifespan-extending properties of calorie restriction [78].

\subsection{Spontaneous and Induced Locomotor Activity}

The spontaneous and induced locomotor activity of flies can be measured to assess fitness, locomotor ability and circadian rhythm.

Already in 1967 Seymour Benzer invented a light countercurrent assay to study locomotor activity and behavioral responses of fruit flies [79]. In a follow-up study, clock genes were discovered in Drosophila, which unraveled important molecular targets of the circadian network [80].

The rapid iterative negative geotaxis (RING) assay, also called the climbing assay, is used for the determination of induced activity (Figure 8). Flies are inserted into vials that are fixed in a holder with a marked target line (RING apparatus). Tapping the RING apparatus makes the flies drop to the bottom of the vials. Negative geotaxis causes flies to have a natural drive to move upward as fast as possible, and the response is more rapid in younger and healthier flies than in older and/or more conspicuously disabled flies [81,82]. Combining negative geotaxis with the video recording of flies enables the identification of smaller differences in locomotor ability [83].

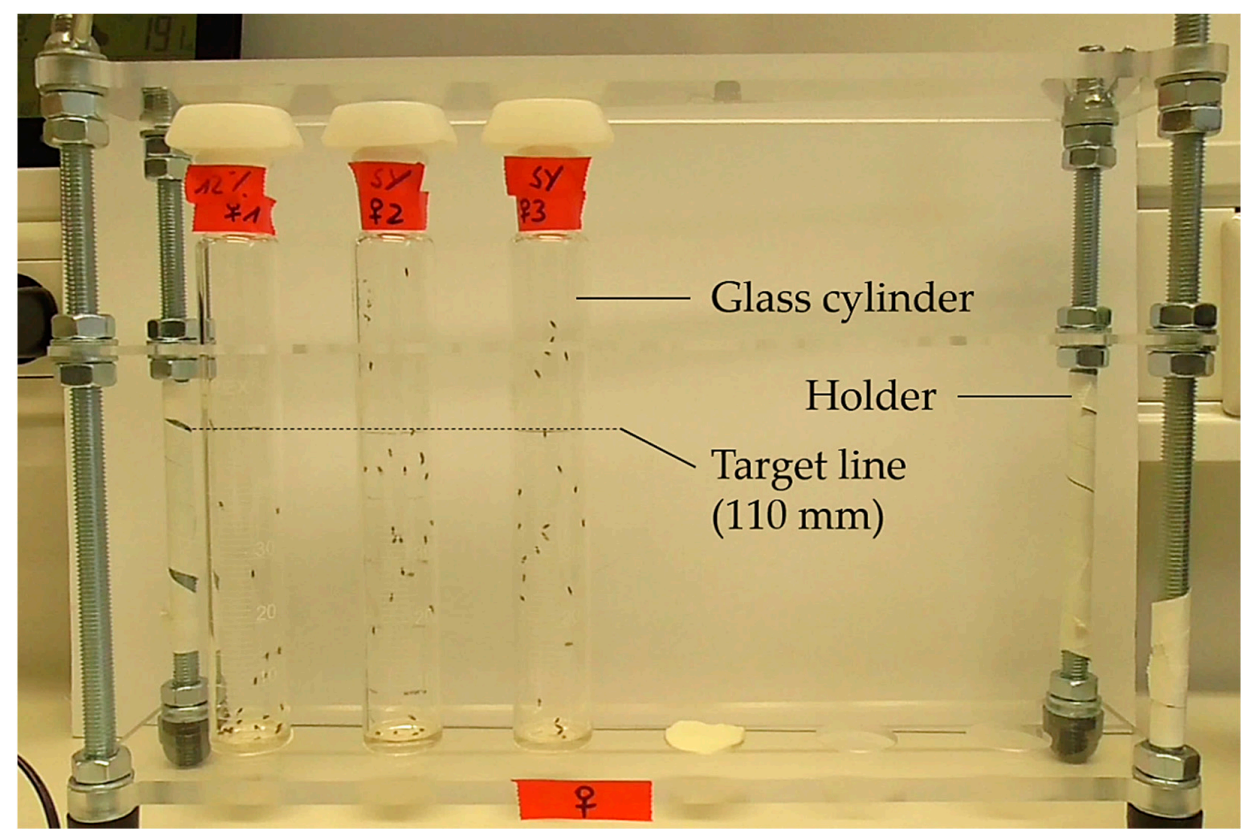

Figure 8. Rapid iterative negative geotaxis (RING) assay. A defined number of flies are inserted into empty vials or cylinders with a target line (marked here at $110 \mathrm{~mm}$ ). The vials or cylinders are fixed in a holder (RING apparatus). After a short adaptation time, the RING apparatus is tapped on the table until all flies are on the bottom of the vials, and a photograph capturing all vials is taken. The process of tapping and photographing is repeated 10 times at 1-min intervals. Alternatively, a video of the climbing process is recorded. These photographs or video recordings are used to determine the climbing ability of the flies (modified from [83]). 
The recording of the spontaneous locomotor activity of $D$. melanogaster enables the analysis of locomotor and sleep patterns and provides data for circadian rhythm assessment. Common techniques are based on video recording or infrared light sensing [84].

Video recording is typically performed in homemade [84-87] or commercially available exposure chambers or arenas. These methods are comparatively inexpensive and allow individual adjustments. Additionally, the recording of individual movement patterns of the fly is possible, and minor defects in the fly's movement can be captured. Recording is restricted by the memory capacity of the recording device [88].

The Drosophila Activity Monitoring (DAM) system (TriKinetics, Figure 9) is based on interruption of an infrared beam [89]. Flies are placed individually or in groups in monitor vials with a continuous food supply. An infrared light sensor is installed centrally in the activity monitor, which generates a signal whenever the sensor is interrupted. The computer-based DAM system provides a simultaneous and continuous recording of the locomotor activity of several flies over several days under standard conditions. However, movement deficits are not detectable in detail, and differentiation among immobility, occupation on the medium and sleep is not possible. Moreover, the locomotion of flies with mobility deficiencies that are unable to cross the light sensor will remain undetected [88].

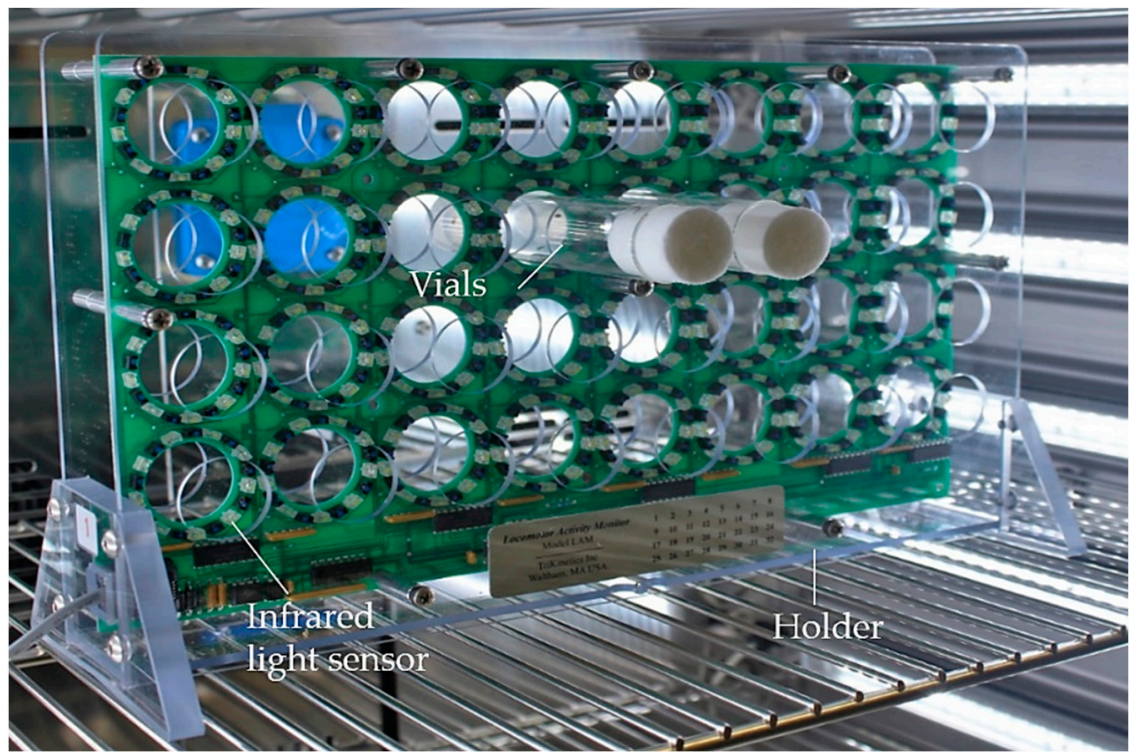

Figure 9. Drosophila Activity Monitoring (DAM) system (TriKinetics). Flies are placed in glass vials that contain experimental food and are covered with plugs. The vials are inserted horizontally into the slots of the holder of the activity monitor. In the center of each slot, an infrared light sensor is installed, which generates a signal whenever the sensor is interrupted by a crossing fly. The activity monitor is linked to a computer, and via the DAM system software, locomotion data from up to 32 vials can be recorded simultaneously (modified from [89]).

\subsection{Heart Rate Measurement}

The beating heart of fruit flies can be monitored by using video microscopy. Initially, flies are anesthetized, fixed, oxygenated saline is added, and surrounding organs are extracted so that the Drosophila heart is exposed. The investigation of parameters such as the heart rate, contractility or cardiac rhythm are measurable $[22,90]$.

\subsection{Fecundity, Fertility and Development as Parameters of Reproduction}

In D. melanogaster, the period of time from maturation to fertility is sex-dependent. In males, the sexual maturation period length is approximately two days [91], and in females, it is about three days post hatch [92]. When females are in regular contact with males, the maximum peak of oviposition is attained after one week, after which the egg numbers 
continuously decline until infertility of the females occurs after approximately 50 days [93]. The important parameters for the characterization of reproduction are fecundity, fertility and development (Figure 10).

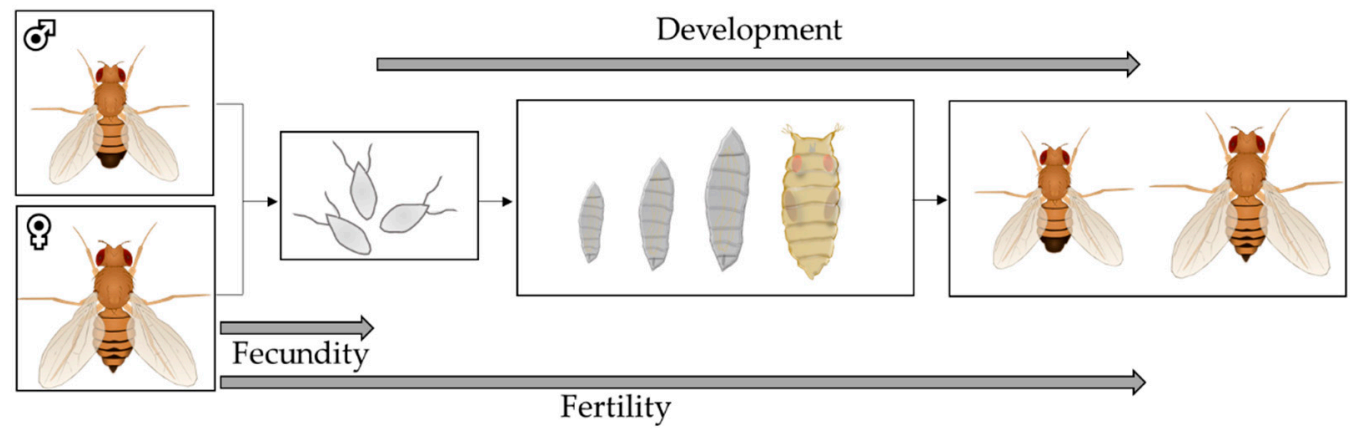

Figure 10. Determination of reproductive parameters in Drosophila melanogaster. After prefeeding males, females or both, single females, a couple or a group of flies are combined for mating. Subsequently, the reproductive performance of the female flies is examined. To determine the fecundity of flies, the eggs laid per time interval are counted. To assess fertility, the number of living offspring (larvae, pupae or adults) that develop from the laid eggs is calculated as a percentage value. Concerning the analysis of the development of the F1 generation, which considers the process from the laid egg through the individual developmental stages to the adult fly, parameters such as the development time or the sex ratio of the offspring are determined (modified from [94]).

Fecundity is defined as the biological capacity for reproduction and is determined in D. melanogaster via the assessment of egg laying in a defined time interval [94]. In nutrition studies, virgin males and females are prefed with a test medium, and then transferred to standard medium for mating [95-97]. To improve egg counting, the medium with laid eggs can be stained with dyes such as Brilliant Blue FCF or rinsed and filtered [98,99]. It is also possible to assess single flies for individual fecundity [100].

Fertility describes the proportion of living offspring from laid eggs [94]. Fertility assessment involves monitoring the development of laid eggs and the documentation of the percentages of larvae, pupae and/or adult flies $[95,97,101]$. The influences of nutritional factors on male and female fecundity and fertility can be determined by different prefeeding and crossbreeding experiments. The effect of nutrition on male fertility, for example, can be determined by prefeeding virgin males with a standard or test medium, subsequently mating them with virgin females prefed with a standard medium and counting the viable offspring [102].

Another aspect of reproduction is the development of the offspring. The whole developmental process from eggs to larvae, pupae and adult flies or partial stages of this process can be considered. Parameters such as the development time, sex ratio, body weight, body composition and fitness of the offspring can be assessed. The consideration of several generations as a long-term approach is also an option [103-105].

\subsection{Stress Assays}

Environmental modifications such as extreme temperature, low food supply, infections or altered oxygen and salt concentrations act as stressors for D. melanogaster and may result in homeostatic imbalance [106]. The impacts of different stressors on the health and fitness parameters of $D$. melanogaster are investigated in laboratory settings (Figure 11), where stress exposure is combined with an additional method for phenotyping, such as determination of the survival rate, climbing activity, fecundity or metabolic analyses. Again, it is important to work with groups of flies of the same sex and age. To study the impact of food on stress tolerance, flies are either prefed with or maintained on experimental diets. 


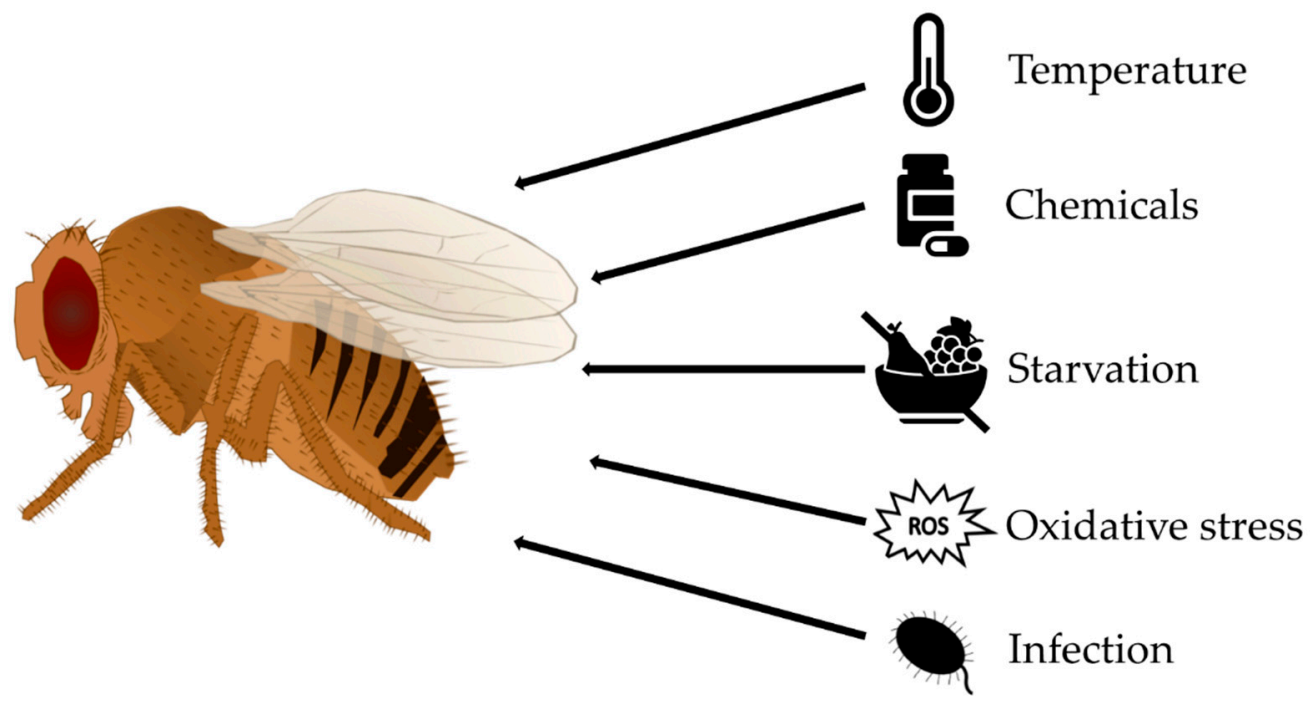

Figure 11. Overview of stress assays established in Drosophila melanogaster. The fruit fly model offers an opportunity to study the impact of dietary interventions such as high-sugar and high-fat diets on the stress tolerance of an organism. To this end, various stress assays were established, in which flies are exposed to high or low temperatures, chemicals, pharmaceuticals, starvation, desiccation or infection with pathogenic microorganisms such as Pseudomonas aeruginosa or Pseudomonas entomophila.

Thermal environment shifts such as extreme heat or cold are physical stressors for ectothermic D. melanogaster [107]. Numerous temperature stress protocols were developed, including short-term exposure (minutes or hours) to lethal or sublethal conditions (the latter termed hardening) as well as long-term exposure (days or weeks) to conditions within the normal viable temperature range of the fruit fly (termed acclimation). Changes in temperature can be applied as ramping temperature stress or sudden stress. A common assay is the heat shock experiment, in which larvae or adults are enclosed in a waterproof vial and placed in a water bath at an elevated temperature (commonly $38-39^{\circ} \mathrm{C}$ ) for a selected time. Immediately following heat exposure, the percentage of flies that exhibit heat-induced paralysis can be determined. Alternatively, after a recovery period, lethality, fecundity, fitness and metabolic function can be investigated [92,108,109].

This experimental setup of a water or ice bath can also be utilized for the opposite thermal conditions of low temperatures [110-112].

Furthermore, the effects of chemical stressors such as pharmaceuticals or high concentrations of heavy metals, such as copper, lead or cadmium, can be studied in D. melanogaster. Heavy metals and other chemical stressors are mainly applied orally as supplements of the diet. The investigation of the impacts of chemical stressors on survival, fertility, heredity and the development of offspring is an example of an application [113,114]. Elevated salt concentrations in the medium elicit osmotic or ionic stress [106].

The influences of starvation or desiccation on D. melanogaster can also be studied. For a starvation assay, larvae or adult flies are transferred to vials containing a liquid source such as an agar plate, wet filter paper or fluid in capillaries or a cup on the bottom $[115,116]$. For a combined starvation and desiccation assay, flies are placed in an empty vial with the optional addition of a desiccant $[106,117]$. Usually, survival rates over time (similar to the case in lifespan assays) or metabolic adaptations are determined.

In addition, $D$. melanogaster is a valuable model organism with which to study tolerance of oxidative stress. Paraquat and hydrogen peroxide are commonly employed for stress treatments. These agents are mixed into the standard medium or applied in a sucrose solution on filter paper [118-120]. The survival rate of flies is monitored over time or determined after a defined period of time.

To study the immune response and tolerance of pathogens, D. melanogaster can be challenged with several bacteria, such as Pseudomonas aeruginosa, Pseudomonas entomophila, 
or Serratia marcescens; viruses, such Drosophila C virus; or fungi, such Aspergillus or Fusarium oxysporum [121-123].

\section{Obesity Phenotype of D. melanogaster Following a High-Sugar or High-Fat Diet}

HSD or HFD feeding induces an obese phenotype with increased fat storage in D. melanogaster [22,124]. In addition, it was demonstrated that the hormones of energy metabolism (e.g., insulin-like peptides (Dilps) (a functional homolog of vertebrate insulin), unpaired2 (a functional homolog of vertebrate leptin), adipokinetic hormone (a functional homolog of vertebrate glucagon)) respond to these dietary interventions in a manner such that obesity comorbidities, e.g., insulin resistance, can be provoked [125]. Accordingly, the fruit fly has become an attractive model for studying the key metabolic processes and phenotypes of obesity and potential comorbidities. Both HSDs and HFDs are characterized by a high energy density and exhibit a low saturation effect and a good sensory quality [125].

In this context, it is of note that the terms "HSD" and "HFD" are generally not defined in terms of the quality and quantity of the added sugar or fat, which complicates the comparison of dietary treatments between studies [4].

\subsection{High-Sugar Diet}

Compared to a standard medium with a usual sucrose content of $5 \%(w / v)$, an HSD contains an elevated amount of sucrose, glucose or fructose, often with a total sugar content of approximately $20-30 \%(w / v)$ [126]. Based on the geometry of nutrition, this results in proportionally lower levels of proteins and fats [127].

Numerous studies have shown that HSD feeding leads to increased glucose, trehalose, glycogen and TAG levels [75,128,129] and decreased protein levels [75] (for details, see Table 3). Furthermore, HSD feeding leads to elevated food intake [75], impairment of carbohydrate homeostasis [130] and insulin resistance [129]. Overall, impacts of single components, such as sugar and yeast, in the medium, as well as the total energy intake, are evident in the phenotypes [75].

The HSD-induced increase in food intake and TAG storage is stimulated by the alteration of satiety behavior and disruption of sweetness detection. An HSD stimulates the hexosamine biosynthesis pathway, causing an accumulation of O-linked $\mathrm{N}$-acetylglucosamine (O-GlcNAc) in the sweet taste neurons, which results in an increased activity of O-GlcNAc transferase, and consequently, a decreased neuronal activity [131]. Additionally, the consumption of an HSD following a fasting period leads to a modification of nutrient utilization, for example, by increasing the activity of the tricarboxylic acid (TCA) cycle [132].

Furthermore, in many publications, it was demonstrated that stress resistance is diminished after the ingestion of an HSD. Extreme cold leads to increased post-cold mortality and extended chill coma recovery time. The sugar concentration and the type of sugar (sucrose, fructose, glucose, trehalose) was observed to influence survival after cold stress [130]. Additionally, the activation of the innate immune system via the Toll and c-Jun $N$-terminal kinase (JNK) signaling pathways could also be induced following ingestion of an HSD [133].

An HSD results in detrimental changes in health and fitness parameters, such as reductions in flying and climbing ability [134] and survivorship [75,135]. Even HSD exposure at a young age induces a long-term decline in lifespan [136].

In this manner, the metabolic effects of an HSD emerge independently of hydration, while the shortening of lifespan following HSD consumption may be reversed by water supplementation. Thus, a shortening of lifespan may not be directly induced by the metabolic consequences of HSD consumption [137].

Alterations at the tissue level are also caused by HSDs. In heart tissue, the dysregulation of the hexosamine biosynthetic pathway leads to increases in hexosamine flux and heart defects, characterized by irregular beating patterns and reduced cardiac contractility [135].

Furthermore, an HSD alters the intestinal tissue, which is associated with the dysfunction of intestinal cell membranes, changes in bacterial composition [138], increased intestinal permeability and the disruption and disorganization of actin filaments in the 
midgut [139]. These alterations are attributed to the elevated differentiation of intestinal stem cells resulting from the upregulation of the c-Jun $\mathrm{N}$-terminal kinase signaling pathway, downregulation of the Janus kinase/signal transducer and activator of transcription signaling pathway [138].

In addition, an HSD is associated with the morphological and functional alteration of the Malpighian tubules, characterized by cytoskeletal deformities, enhanced oxidative stress, apoptosis [140], increased deposition of uric acid stones in the Malpighian tubules and hindgut and reduced tubule secretion rates [137]. This state is linked to interference with purine biosynthesis, resulting in enhanced uric acid production, the generation of a dehydration state and acidification of the tubules and the intestine [137].

Moreover, an HSD causes neurodegenerative modifications of the eyes, e.g., increased disorganization of ommatidia, enhanced apoptosis and negative alterations in autophagy, which are partially caused by oxidative stress via ROS. Consequently, deficits in light sensitivity and visual disturbances are increasingly evident [141].

In addition, several studies indicated that decreases in reproductive capacity [142] and genetic programming $[128,143]$ are consequences of HSD consumption. Overnutrition of the maternal F0 generation affects the predisposition for the development of an overweight phenotype under conditions of high food supply in the offspring. L3 male larvae of the F1 and F2 generations have increased whole-body glucose and trehalose levels, while females of the F2 generation exhibit increased trehalose levels and decreased TAG levels. Modifications including gene expression alterations suggest that changes in carbohydrate homeostasis and nutrient storage occur in subsequent generations [128].

In males, HSD feeding modifies genetic programming, which influences the metabolism of the subsequent F1 generation. Males of the F1 generation are predisposed to an obesity phenotype and exhibit increased body weight and TAG storage when fed an HSD compared to a normal diet. Additionally, the adipose area and lipid droplet sizes are enhanced, food intake is increased, and the starvation sensitivity of males of the F1 generation is elevated. However, no effect of the paternal diet on the development time, offspring quantity or sex ratio is observed [143].

\subsection{High-Fat Diet}

Similar to HSDs, HFDs can also be applied to generate an obesity phenotype of D. melanogaster. To create an HFD, an additional fat source, such as $20-30 \%(w / v)$ coconut oil or $15 \%(w / v)$ lard, is added to the normally low-fat standard medium [126].

Numerous studies demonstrated that an HFD causes increased TAG, glucose, and trehalose levels and alterations in glucose and insulin homeostasis $[8,22,110,134]$ (for details, see Table 3). An HFD was shown to have a more severe impact than an HSD [134]. However, an assessment of the mating state of females indicates an interaction with the HFD-induced alteration in body composition, as effects are not observed in virgin females [144].

An HFD contributes to obesity via metabolic changes accompanied by modifications of sensory cognition and the perception of hunger and satiety $[145,146]$. Sensory cognition is mediated by a reduction in DmOrco expression in the peripheral olfactory organ and modification of gene expression in antennae, e.g., with regard to the olfactory receptors and insulin signaling pathway [146]. Additionally, an HFD provokes modifications in the central nervous system, such as the suppression of neuronal autophagy in octopaminergic neurons, resulting in the modification of hunger and satiety perceptions [147]. 
Table 3. Overview of dietary intervention studies regarding high-sugar diet (HSD) or high-fat diet (HFD) feeding in Drosophila melanogaster. The studies are ordered by release date.

\begin{tabular}{|c|c|c|c|c|c|}
\hline Diet & Strain, Sex, Age & Time Period of Intervention & $\begin{array}{c}\text { Assays (Number of Larvae or Flies } \\
\text { per Treatment or } \\
\mathbf{n}=\text { Number of Treatments) }\end{array}$ & Outcomes & Publication \\
\hline \multicolumn{6}{|l|}{ HSD } \\
\hline $\begin{array}{l}\text { Combination of different } \\
\text { sugar and yeast ratios } \\
\text { HSD } \\
2.5-40 \% \text { sugar } \\
2.5-40 \% \text { yeast } \\
1.5 \% \text { agar } \\
1.8 \% \text { fungicide }\end{array}$ & $\begin{array}{l}y w ; w^{1118} ; \text { Canton-S } \\
\text { Males, females } \\
1 \mathrm{~d}\end{array}$ & $\begin{array}{l}\text { Prefeeding } \\
3 \mathrm{~d} 10 \% \text { sugar/yeast } \\
13 \mathrm{~d}, 26-40 \mathrm{~d}, \\
52-56 \mathrm{~d}\end{array}$ & $\begin{array}{ll}\text { - } & \text { Food intake } \\
\text { - } & \text { (80 females/50 males) } \\
\text { Body composition } \\
\text { TAG } \\
\text { Proteins } \\
\text { (80 females/50 males) } \\
\text { Lifespan } \\
\text { (>250 females) } \\
\text { Fecundity }(80)\end{array}$ & $\begin{array}{ll}\text { - } & \text { Food intake } \uparrow \\
\text { - } & \text { Body composition } \\
& \text { TAGs } \uparrow \\
& \text { Protein storage } \downarrow \\
\text { - } & \text { Lifespan } \downarrow \\
\text { - } & \text { Fecundity } \downarrow \\
\text { - } & \text { Amplification of effect with } \\
& \text { age }\end{array}$ & Skorupa et al. [75] \\
\hline $\begin{array}{l}\text { Control diet } \\
5 \% \text { sugar } \\
10 \% \text { yeast } \\
2 \% \text { peptone } \\
1 \% \text { agar } \\
\text { HSD } \\
34 \% \text { sugar }\end{array}$ & $\begin{array}{l}\text { Canton-S } \\
\text { L3 larvae }\end{array}$ & $\begin{array}{l}\text { Shortterm: } \\
12 \mathrm{~h} \\
\text { or } \\
\text { Longterm: Egg to L3 } \\
\text { Wandering L3 stage }\end{array}$ & $\begin{array}{ll}\text { - } & \text { Food intake } \\
\text { Body size, } \\
\text { - } \quad \text { body weight }(>15) \\
\text { Body composition } \\
\text { Hemolymph: } \\
\text { Glucose }(>160) \\
\text { Trehalose }(>15) \\
\text { Whole body: } \\
\text { TAG } \\
\text { Carbohydrates }(>25 \text { larvae, }>30 \\
\text { flies }) \\
\text { Development }(n=3) \\
\text { Molecular analysis } \\
\text { qRT-PCR }(n=7) \\
\text { Western blot }(n>4) \\
\text { Lipid droplet measurement } \\
\text { (>15) } \\
\text { Microarrays }\end{array}$ & 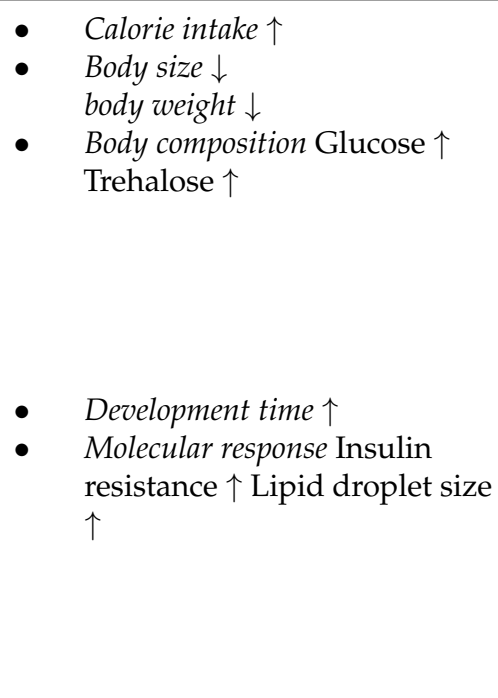 & $\begin{array}{l}\text { Palanker Musselman } \\
\text { et al. [129] }\end{array}$ \\
\hline
\end{tabular}


Table 3. Cont.

\begin{tabular}{|c|c|c|c|c|c|}
\hline Diet & Strain, Sex, Age & Time Period of Intervention & $\begin{array}{c}\text { Assays (Number of Larvae or Flies } \\
\text { per Treatment or } \\
\mathbf{n}=\text { Number of Treatments) }\end{array}$ & Outcomes & Publication \\
\hline $\begin{array}{l}\text { - Control diet } \\
10,400 \mathrm{mM} \text { sucrose, } \\
\text { fructose, glucose or } \\
\text { trehalose } \\
8 \% \text { yeast } \\
1.5 \% \text { agar } \\
\text { HSD } \\
1000 \mathrm{mM} \text { sucrose, } \\
\text { fructose, glucose or } \\
\text { trehalose }\end{array}$ & $\begin{array}{ll}\text { - } & \text { Mix of two wild } \\
& \text { populations } \\
\text { - } & \text { Females } \\
\text { - } & 5 \mathrm{~d}\end{array}$ & - $\quad$ Egg to $5 \mathrm{~d}$ adult females & $\begin{array}{ll}\text { - } & \text { Body weight } \\
\text { - } & \text { Development } \\
\text { - } & \text { Stress assay } \\
& \text { Cold tolerance assays: } \\
\text { nonlethal chronic cold: } \\
0^{\circ} \mathrm{C}, 16 \mathrm{~h}(50) \text { acute cold stress: } \\
-3.5^{\circ} \mathrm{C}, 2 \mathrm{~h}(100) \\
\text { Body composition } \\
\text { TAG (50) } \\
\text { - } \text { Molecular analysis } \\
\text { Metabolic fingerprinting }(90)\end{array}$ & $\begin{array}{ll}\text { Results for sucrose: } \\
-\quad \text { Development time } \uparrow \\
-\quad \text { Stress tolerance } \downarrow \\
\text { - } \quad \text { Body composition } \\
\text { TAGs } \uparrow \\
\text { Sorbitol } \uparrow \\
\text { Sucrose } \uparrow \\
\text { Fructose } \uparrow \\
\text { Inositol } \uparrow \\
\text { Malate } \uparrow \\
\text { Xylitol } \uparrow\end{array}$ & Colinet et al. [130] \\
\hline & & & & $\begin{array}{ll}\text { Results for fructose: } \\
\text { - } \quad \text { Body weight } \uparrow \\
\text { - } & \text { Development time } \uparrow \\
\text { - } & \text { Stress tolerance } \downarrow \\
\text { - } & \text { Body composition } \\
\text { TAGs } \uparrow \\
\text { Malate } \uparrow \\
\text { Fructose } \uparrow \\
\text { Sorbitol } \uparrow \\
\text { Trehalose } \uparrow \\
\text { Inositol } \uparrow\end{array}$ & \\
\hline
\end{tabular}


Table 3. Cont.

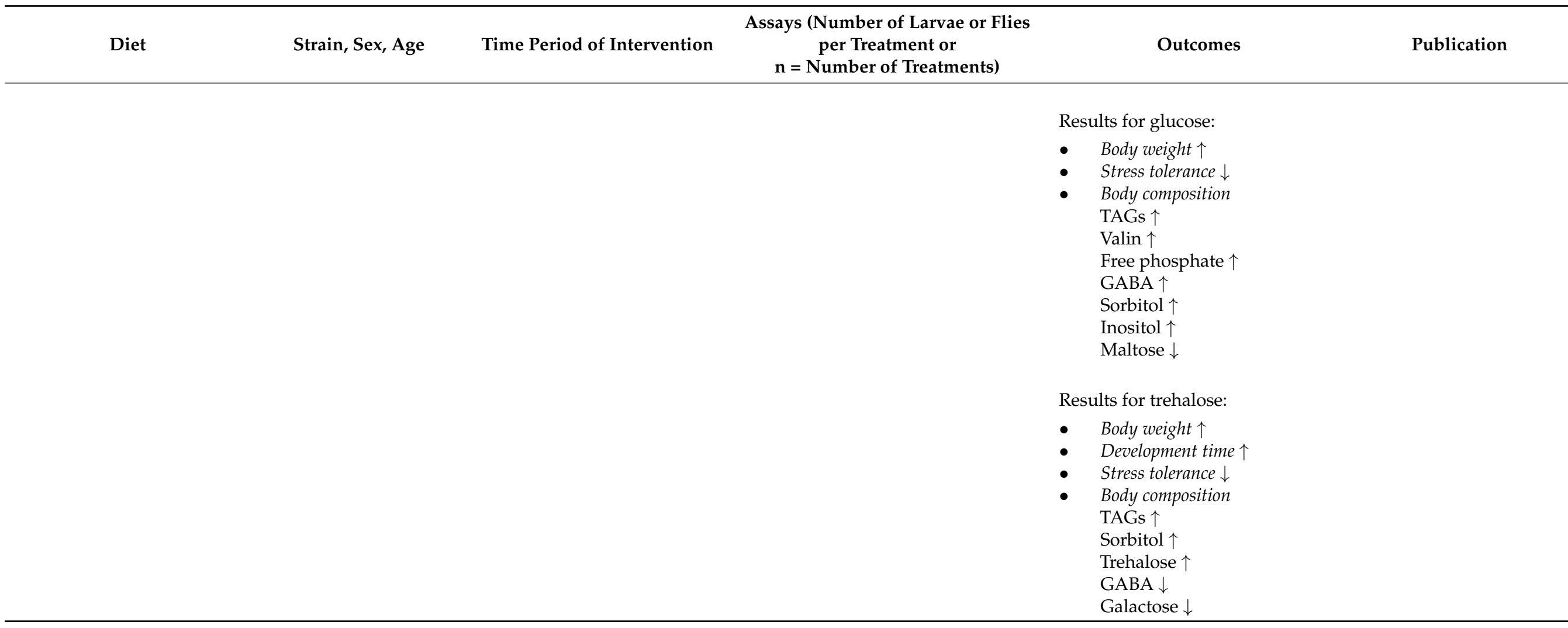


Table 3. Cont.

\begin{tabular}{|c|c|c|c|c|c|}
\hline Diet & Strain, Sex, Age & Time Period of Intervention & $\begin{array}{c}\text { Assays (Number of Larvae or Flies } \\
\text { per Treatment or } \\
\mathbf{n}=\text { Number of Treatments) }\end{array}$ & Outcomes & Publication \\
\hline $\begin{array}{l}\text { Control diet } \\
5 \% \text { sucrose } \\
10 \% \text { yeast } \\
2 \% \text { peptone } \\
1 \% \text { agar } \\
\text { HSD } \\
34 \% \text { sucrose }\end{array}$ & $\begin{array}{l}w^{1118} \\
\text { L3 larvae } \\
\text { Females }\end{array}$ & $\begin{array}{l}\text { Virgin females } \\
7 \mathrm{~d}\end{array}$ & $\begin{array}{ll}\text { - } & \text { Body weight } \\
\text { Body composition } \\
\text { Whole body: } \\
\text { Glucose } \\
\text { Trehalose } \\
\text { Glycogen } \\
\text { (>150 larvae/>160 flies) } \\
\text { Hemolymph: } \\
\text { Glucose } \\
\text { Trehalose } \\
\text { (>160) } \\
\text { Molecular analysis } \\
\text { Real-time quantitative PCR } \\
\text { (qRT-PCR, }>10 \text { pooled samples) }\end{array}$ & $\begin{array}{l}\text { Females: } \\
\text { - } \quad \text { Body weight } \downarrow \\
\text { - } \quad \text { Body composition } \\
\text { Whole body: } \\
\text { Trehalose } \uparrow \\
\text { Glycogen } \uparrow \\
\text { TAGs } \uparrow\end{array}$ & Buescher et al. [128] \\
\hline $\begin{array}{l}\text { Control diet } \\
5 \% \text { sucrose } \\
10 \% \text { yeast } \\
2 \% \text { peptone } \\
1 \% \text { agar } \\
\text { magnesium sulfate calcium } \\
\text { chloride } \\
\text { HSD } \\
34 \% \text { sucrose }\end{array}$ & $\begin{array}{l}w^{1118} \\
\text { Males } \\
\text { After eclosion }\end{array}$ & 3 weeks & $\begin{array}{ll}\text { - } & \text { Body composition TAG (12) } \\
\text { Hemolymph: } \\
\text { Glucose } \\
\text { Trehalose } \\
\text { (35-45) } \\
\text { - } \quad \text { Lifespan (50) } \\
\text { Development } \\
\text { - Molecular analysis } \\
\text { Western blot }(n=10) \\
\text { Optical heartbeat analysis } \\
\text { Fluorescence staining, } \\
\text { imaging }(n=3)\end{array}$ & $\begin{array}{ll}\text { - } & \text { Body composition } \\
\text { TAGs } \uparrow \\
\text { Glucose } \uparrow \\
\text { Trehalose } \uparrow \\
\\
\text { - } \quad \text { Lifespan } \downarrow \\
\text { - } \quad \text { Development time } \uparrow \\
\text { Molecular response } \\
\text { Insulin resistance } \uparrow \\
\text { Hexosamine flux } \uparrow \\
\text { Heart: } \\
\text { Irregular beating patterns } \uparrow \\
\text { Fibrillations } \uparrow \\
\text { Asystolic periods } \uparrow \\
\text { Heart contractility } \downarrow \\
\text { Heart TAGs } \uparrow \\
\text { Heart collagen } \uparrow \\
\text { Mortality } \uparrow\end{array}$ & Na et al. [135] \\
\hline
\end{tabular}


Table 3. Cont.

\begin{tabular}{|c|c|c|c|c|c|}
\hline Diet & Strain, Sex, Age & Time Period of Intervention & $\begin{array}{c}\text { Assays (Number of Larvae or Flies } \\
\text { per Treatment or } \\
\mathbf{n}=\text { Number of Treatments) }\end{array}$ & Outcomes & Publication \\
\hline $\begin{array}{l}\text { Paternal diet: } \\
\text { Control diet } \\
3 \% \text { sucrose } \\
1 \% \text { yeast } \\
3 \% \text { soy flour } \\
1.2 \% \text { agar } \\
\text { HSD } \\
10 \text { or } 30 \% \text { sucrose } \\
\text { F1 flies: } \\
1.8 \% \text { yeast } \\
1 \% \text { soy flour } \\
8 \% \text { yellow corn meal } \\
2.2 \% \text { molasses } \\
8 \% \text { malt extract } \\
1.2 \% \text { agar }\end{array}$ & $\begin{array}{l}w^{1118} \\
\text { Males } \\
4-5 \mathrm{~d}\end{array}$ & $\begin{array}{l}\text { Parental flies } 2 \mathrm{~d} \text { on HSD } \\
\text { Investigation of F1 flies } 7 \mathrm{~d} \\
\text { post eclosion }\end{array}$ & $\begin{array}{ll}\text { - } & \text { Body composition } \\
\text { TAG } \\
\text { Glucose } \\
\text { Trehalose } \\
\quad(>15) \\
\text { - } \quad \text { Wing size }(n>3) \\
\text { - Food intake } \\
\text { - } \quad \text { Metabolic rate }(n>3) \\
\text { - } \quad \text { Despirometer }(>15) \\
\text { Development }(25) \\
\text { Molecular analysis } \\
\text { Eye pigment }(>15) \\
\text { Sperm dissection }(25) \text { and RNA } \\
\text { isolation } \\
\text { Fat bodies: } \\
\text { Immunofluorescence } \\
\text { ( } n=7) \\
\text { RNA sequencing } \\
\text { Bioinformatic analysis }\end{array}$ & - $\quad$ Body composition TAGs $\uparrow$ & Öst et al. [143] \\
\hline
\end{tabular}


Table 3. Cont.

\begin{tabular}{|c|c|c|c|c|c|}
\hline Diet & Strain, Sex, Age & Time Period of Intervention & $\begin{array}{c}\text { Assays (Number of Larvae or Flies } \\
\text { per Treatment or } \\
\mathbf{n}=\text { Number of Treatments) }\end{array}$ & Outcomes & Publication \\
\hline $\begin{array}{l}\text { Control diet } \\
5 \% \text { sucrose } \\
10 \% \text { yeast } \\
2 \% \text { peptone } \\
1 \% \text { agar } \\
\text { HSD } \\
34 \% \text { sucrose }\end{array}$ & $\begin{array}{l}w^{1118} \\
\text { Females } \\
1 \mathrm{~d}\end{array}$ & $\begin{array}{l}\text { Virgin females } 7 \mathrm{~d} \text { on HSD } \\
\text { F1 adult males } 14 \mathrm{~d} \text { on HSD }\end{array}$ & $\begin{array}{ll}\text { - } & \text { Body length }(5) \\
\text { Ovarian area, } \\
\text { ovarian length, } \\
\text { ovarian width } \\
\text { (83-93) } \\
\text { Fecundity } \\
\text { Stage } 14 \text { eggs }(40) \\
\text { Development }(>9 / \text { development } \\
\text { stage) } \\
\text { Ovarian composition } \\
\text { TAG } \\
\text { Glucose } \\
\text { Cholesterol } \\
\text { (>250) } \\
\text { Molecular analysis } \\
\text { Insulin sensitivity }(>30) \\
\text { qRT-PCR: } \\
\text { Heads }(40) \\
\text { Ovaries }(>48) \\
\text { L3 male larvae }(40) \\
\text { Protein carbonylation }(>30)\end{array}$ & $\begin{array}{l}\text { - } \quad \begin{array}{l}\text { Ovarian area } \downarrow \\
\text { ovarian width } \downarrow\end{array} \\
\text { - } \quad \begin{array}{l}\text { Fecundity } \\
\text { Egg number } \downarrow\end{array} \\
\text { - } \quad \begin{array}{l}\text { Ovarian composition } \\
\text { TAGs } \uparrow\end{array} \\
\text { Cholesterol } \uparrow \\
\text { Molecular response } \\
\text { Insulin resistance } \uparrow \\
\text { Insulin production } \downarrow \\
\text { Oxidative stress } \uparrow \\
\text { Alteration of mitochondrial } \\
\text { gene profile }\end{array}$ & Brookheart et al. [142] \\
\hline $\begin{array}{l}\text { Control diet } \\
5 \% \text { sucrose } \\
10 \% \text { yeast } \\
1.5 \% \text { agar } \\
\\
\text { HSD } \\
40 \% \text { sucrose }\end{array}$ & $\begin{array}{l}w^{1118} \\
\text { Females } \\
2 \mathrm{~d}\end{array}$ & $\begin{array}{l}1-3 \text { weeks, followed by } 1 \\
\text { week on control diet }\end{array}$ & $\begin{array}{l}\text { - } \quad \text { Lifespan (403) } \\
\\
\text { - } \quad \text { Molecular analysis } \\
\text { RNA sequencing (RNA-seq) } \\
\text { qRT-PCR } \\
\text { Western blot } \\
\text { S-adenosylmethionine ELISA } \\
\text { 4'6-Diamidino-2-phenylindole } \\
\text { staining (50-90 nuclei) }\end{array}$ & $\begin{array}{l}\text { - } \begin{array}{l}\text { Lifespan } \downarrow \\
\text { Detrimental effects in mid-life } \\
\text { and late life }\end{array} \\
\text { Molecular response } \\
\text { FOXO phosphorylation } \uparrow \\
\text { Insulin-like } \\
\text { signaling } \uparrow\end{array}$ & Dobson et al. [136] \\
\hline
\end{tabular}


Table 3. Cont.

\begin{tabular}{|c|c|c|c|c|c|}
\hline Diet & Strain, Sex, Age & Time Period of Intervention & $\begin{array}{c}\text { Assays (Number of Larvae or Flies } \\
\text { per Treatment or } \\
\mathbf{n}=\text { Number of Treatments) }\end{array}$ & Outcomes & Publication \\
\hline \multirow{3}{*}{$\begin{array}{l}\text { Control diet } \\
\text { (cornmeal/yeast fly } \\
\text { medium) }\end{array}$} & $w^{1118}$ & \multirow[t]{20}{*}{$5-7 d$} & - Intestinal microbiome & - Intestinal microbiome & \multirow[t]{20}{*}{ Zhang et al. [138] } \\
\hline & Females & & & composition & \\
\hline & & & & Growth rate $\uparrow$ & \\
\hline \multirow{17}{*}{$\begin{array}{l}\text { HSD } \\
+1 \mathrm{M} \text { sucrose }\end{array}$} & $5-7 d$ & & - $\quad$ Molecular analysis & Bacterial number and & \\
\hline & & & Immunostaining assays & density $\downarrow$ & \\
\hline & & & $(7-A A D)$ & p-JNK levels $\uparrow$ & \\
\hline & & & Dihydroethidium (DHE) & lacZp cells $\uparrow$ & \\
\hline & & & staining (gut; $n=3$ ) & Downregulation of & \\
\hline & & & RNA sequencing & JAK/STAT & \\
\hline & & & & Reactive oxygen species & \\
\hline & & & & levels $\uparrow$ & \\
\hline & & & & Cell death $\uparrow$ & \\
\hline & & & & Intestine: & \\
\hline & & & & Disruption of gut homeostasis & \\
\hline & & & & Differentiation of intestinal & \\
\hline & & & & stem cell (ISCs) $\uparrow$ & \\
\hline & & & & Midgut diameter $\downarrow$ & \\
\hline & & & & Disorganization of gut cells & \\
\hline & & & & Disruption of gut cell & \\
\hline & & & & membrane function & \\
\hline
\end{tabular}


Table 3. Cont.

\begin{tabular}{|c|c|c|c|c|c|}
\hline Diet & Strain, Sex, Age & Time Period of Intervention & $\begin{array}{c}\text { Assays (Number of Larvae or Flies } \\
\text { per Treatment or } \\
\mathbf{n}=\text { Number of Treatments) }\end{array}$ & Outcomes & Publication \\
\hline $\begin{array}{l}\text { Control diet } \\
5 \% \text { sucrose } \\
8 \% \text { yeast } \\
2 \% \text { yeast extract } \\
2 \% \text { peptone } \\
1 \% \text { agar } \\
\text { HSD } \\
34 \% \text { sucrose }(1 \mathrm{M})\end{array}$ & $\begin{array}{l}w^{1118} \\
\text { Females } \\
3 \mathrm{~d}\end{array}$ & 3 weeks & $\begin{array}{ll}\text { - } & \text { Lifespan (945) } \\
\text { - } & \text { Food intake } \\
\text { - } & \text { Body weight (5) } \\
\text { Molecular analysis } \\
\text { Smurf (945) } \\
\text { Alkaline phosphatase (5) } \\
\text { Imaging of midgut (3) } \\
\text { Immunohistochemistry of } \\
\text { fly gut } \\
\text { Intestinal and bacterial } \\
\text { cell cultures }\end{array}$ & $\begin{array}{ll}- & \text { Food intake } \uparrow \\
\text { - } & \text { Body weight } \downarrow \\
\text { Molecular response, pathology } \\
\text { Intestine: } \\
\text { Gut permeability } \uparrow \\
\text { Intestinal alkaline } \\
\text { phosphatase } \\
\text { activity } \downarrow \\
\text { Gut length and diameter } \downarrow \\
\text { Broken and disorganized } \\
\text { actin filaments in } \\
\text { posterior midgut }\end{array}$ & Pereira et al. [139] \\
\hline $\begin{array}{l}\text { Control diet } \\
\text { sucrose }(0.15 \mathrm{M}) \\
\text { cornmeal/yeast medium } \\
\text { HSD } \\
\text { sucrose }(1 \mathrm{M})\end{array}$ & $\begin{array}{l}w^{1118} \\
\text { Post-eclosion } \\
\text { L3 larvae }\end{array}$ & $3-5 d$ & $\begin{array}{l}\text { Molecular analysis } \\
\text { Larval and adult phagocytosis } \\
\text { (15-20) } \\
\text { F-actin (5-10 larvae) } \\
\text { Hemocyte (10 larvae) } \\
\text { Immunostaining (lymph } \\
\text { glands, fat bodies, 5-10 larvae) } \\
\text { Terminal deoxynucleotidyl } \\
\text { transferase dUTP nick end } \\
\text { labeling (TUNEL) } \\
\text { 7-AAD staining } \\
\text { (10 larvae) } \\
\text { qRT-PCR (10 larvae) }\end{array}$ & $\begin{array}{l}\text { Molecular response, } \\
\text { pathology } \\
\text { Cell structure: } \\
\text { Larval and adult } \\
\text { phagocytosis } \downarrow \\
\text { Larvae: } \\
\text { Disrupted filaments of } \\
\text { plasmatocytes and F-actin } \\
\text { cytoskeleton crystal cells } \downarrow \\
\text { Lamellocytes } \uparrow \text { in } \\
\text { lymph glands } \\
\text { Immune system: activation of } \\
\text { Toll and JNK } \\
\text { signaling pathways } \\
\text { TUNEL- and 7-AAD-positive } \\
\text { cells in fat body } \uparrow\end{array}$ & Yu et al. [133] \\
\hline
\end{tabular}


Table 3. Cont.

\begin{tabular}{|c|c|c|c|c|c|}
\hline Diet & Strain, Sex, Age & Time Period of Intervention & $\begin{array}{c}\text { Assays (Number of Larvae or Flies } \\
\text { per Treatment or } \\
\mathbf{n}=\text { Number of Treatments) }\end{array}$ & Outcomes & Publication \\
\hline $\begin{array}{l}\text { Control diet } \\
5 \% \text { sucrose } \\
7.5 \% \text { white corn } \\
\text { syrup } \\
2 \% \text { yeast } \\
1 \% \text { soy flour } \\
7 \% \text { cornmeal } \\
1 \% \text { agar } \\
\text { HSD: } \\
20 \text { or } 30 \% \text { sucrose }\end{array}$ & $\begin{array}{l}w^{1118} \\
\text { Males } \\
2-5 \mathrm{~d} ; 6-8 \mathrm{~d} \text { (RNAi } \\
\text { lines) }\end{array}$ & 2 and $7 \mathrm{~d}$ & $\begin{array}{l}\text { - Food intake } \\
\text { PER (22-61) } \\
\text { FLIC (16-72) } \\
\text { Optogenetic stimulation } \\
\text { for FLIC } \\
\text { Taste sensitivity } \\
\\
\text { - Body composition } \\
\text { TAG (24) } \\
\text { Protein (3/replicate) } \\
\text { Molecular and neuronal analysis } \\
\text { Nile red staining (fat bodies of } 3 \\
\text { flies) } \\
\text { Sensillar electrophysiology } \\
\text { Metabolomics (100 heads) } \\
\text { Calcium imaging (26 brains) } \\
\text { RNA sequencing } \\
\text { Proboscis immunofluorescence } \\
\text { qRT-PCR (40-50 heads) }\end{array}$ & $\begin{array}{l}\text { - Food intake } \\
\text { PE } \downarrow \text { over time } \\
\text { Behavioral responses to } \\
\text { food } \downarrow \\
\text { Sweetness } \\
\text { sensitivity } \downarrow \\
\text { Meal duration and size over } \\
\text { time } \uparrow \\
\text { Modification of satiety but } \\
\text { not hunger } \\
\text { Body composition } \\
\text { Fat } \uparrow \\
\text { Molecular and neuronal response } \\
\text { Taste neurons: } \\
\text { Presynaptic responses over } \\
\text { time } \downarrow \\
\text { Stimulation of hexosamine } \\
\text { biosynthetic pathway (HBP) } \\
\text { O-GlcNAc } \uparrow \\
\text { Activity O-GlcNAc } \\
\text { transferase (OGT) } \uparrow \\
\text { Genes encoding proteins with } \\
\text { neural function- and } \\
\text { metabolism-related activity, } \\
\text { e.g., glutaminase and } \\
\text { carbonate dehydratase }\end{array}$ & May et al. [131] \\
\hline
\end{tabular}


Table 3. Cont.

\begin{tabular}{|c|c|c|c|c|c|}
\hline Diet & Strain, Sex, Age & Time Period of Intervention & $\begin{array}{c}\text { Assays (Number of Larvae or Flies } \\
\text { per Treatment or } \\
\mathbf{n}=\text { Number of Treatments) }\end{array}$ & Outcomes & Publication \\
\hline $\begin{array}{l}\text { Control diet } \\
8 \% \text { sucrose } \\
0.5 \% \text { yeast } \\
2 \% \text { agar } \\
0.16 \% \text { calcium chloride } \\
\text { dihydrate } \\
0.16 \% \text { ferrous sulfate } \\
\text { heptahydrate } 0.8 \% \text { sodium } \\
\text { potassium tartrate } \\
\text { tetrahydrate } \\
0.05 \% \text { sodium chloride } \\
0.05 \% \text { manganese chloride } \\
\text { tetrahydrate } \\
0.53 \% \text { nipagin } \\
\text { HSD } \\
+300 \mathrm{mM} \text { sucrose }\end{array}$ & $\begin{array}{l}\text { Canton-S; } w^{1118} \\
\text { Males, females } \\
1,3,5 \text { weeks }\end{array}$ & $4 \mathrm{~d}$ & $\begin{array}{ll}\text { - } & \text { Body weight } \\
\text { Body composition } \\
\text { TAG } \\
(>30) \\
\text { Hemolymph: } \\
\text { Glucose } \\
\text { Trehalose }(>100) \\
\text { - Locomotion activity } \\
\text { Climbing }(>100) \\
\text { Activity }(50) \\
\text { Flight }(>100) \\
\text { Food intake } \\
\text { CAFE }(>50) \\
\text { Lifespan }(100) \\
\text { Starvation }(50) \\
\text { Molecular analysis } \\
\text { Bromoenol lactone treatment } \\
\text { (100) } \\
\text { Cytological analysis of muscle } \\
\text { and adipose tissues } \\
\text { Ultrastructural analysis of } \\
\text { muscle }(n>3) \\
\text { qRT-PCR }(n>3) \\
\text { Western blot }(10)\end{array}$ & $\begin{array}{ll}\text { - } & \text { Body weight } \uparrow \\
\text { Body composition } \\
\text { TAGs } \uparrow\end{array}$ & Villanueva et al. [134] \\
\hline
\end{tabular}


Table 3. Cont.

\begin{tabular}{|c|c|c|c|c|c|}
\hline Diet & Strain, Sex, Age & Time Period of Intervention & $\begin{array}{c}\text { Assays (Number of Larvae or Flies } \\
\text { per Treatment or } \\
\mathbf{n}=\text { Number of Treatments) }\end{array}$ & Outcomes & Publication \\
\hline $\begin{array}{l}\text { Control diet } \\
7.5 \% \text { white corn } \\
\text { syrup } \\
1.7 \% \text { yeast } \\
1 \% \text { soy flour } \\
7 \% \text { cornmeal } \\
0.7 \% \text { agar } \\
0.9 \% \text { propionic acid } \\
0.4 \% \text { tegosept } \\
\text { HSD } \\
+30 \% \text { sugar }\end{array}$ & $\begin{array}{l}w^{1118} \\
\text { Males } \\
2-4 \mathrm{~d}\end{array}$ & $\begin{array}{l}18-24 \mathrm{~h} \text { fasting or HSD } \\
\text { feeding (refeeding) }\end{array}$ & $\begin{array}{ll}\text { - } & \text { Body composition } \\
\text { TAG (24-28) } \\
\text { Food intake } \\
\text { FLIC (30) } \\
\text { Foraging (24-28) } \\
\\
\text { - } \quad \text { Molecular analysis } \\
\text { Metabolomics (300 heads; } \\
\text { 150 bodies) } \\
\text { RNA sequencing (10 brains) }\end{array}$ & $\begin{array}{l}\text { - Food intake } \\
\text { FLIC feeding interaction } \downarrow \\
\text { Motivation of feeding } \downarrow \\
\\
\text { - } \quad \text { Metabolic response } \\
\text { Citric acid cycle activity } \uparrow \\
\text { 1-Carbon metabolism } \downarrow \\
N \text {-acetyl-aspartate } \downarrow \\
\text { Kynurenine } \downarrow \\
\text { Glucose usage } \uparrow \\
\text { Neuronal response GABA } \uparrow \\
\text { Glutamate } \uparrow \\
\text { Choline } \uparrow \\
N \text {-acetylserotonin } \uparrow \\
\text { Alteration of neurochemicals } \\
\text { Metabolomics } \\
\text { Changes in } 54 / 377 \\
\text { compounds (e.g., fumarate } \uparrow, \\
\text { malate } \uparrow \text { ) }\end{array}$ & Wilinski et al. [132] \\
\hline
\end{tabular}


Table 3. Cont.

\begin{tabular}{|c|c|c|c|c|c|}
\hline Diet & Strain, Sex, Age & Time Period of Intervention & $\begin{array}{c}\text { Assays (Number of Larvae or Flies } \\
\text { per Treatment or } \\
\mathbf{n}=\text { Number of Treatments) }\end{array}$ & Outcomes & Publication \\
\hline $\begin{array}{l}\text { Control diet } \\
15 \mathrm{~g} \text { sugar }(0.15 \mathrm{M}) \\
6 \mathrm{~g} \text { yeast } \\
17 \mathrm{~g} \text { maize } \\
1.5 \mathrm{~g} \text { agar } \\
1 \mathrm{~mL} \text { propionic acid } 1 \mathrm{~g} \\
\text { methyl-paraben } \\
\mathrm{HSD} \\
1 \mathrm{M} \text { sucrose }\end{array}$ & $\begin{array}{l}\text { Oregon-R } \\
\text { Male } \\
1 \mathrm{~d}\end{array}$ & $5,10,15,20$ and $25 \mathrm{~d}$ & $\begin{array}{l}\text { - } \quad \text { Lifespan (200) } \\
\text { Body composition } \\
\text { Hemolymph: } \\
\text { Glucose } \\
\text { Trehalose } \\
\text { (30) } \\
\text { Whole body: } \\
\text { TAG } \\
\text { Protein } \\
\text { (30) } \\
\text { Molecular analysis } \\
\text { Uric acid (30) } \\
\text { Images } \\
\text { Immunohistochemistry } \\
\text { F-actin staining } \\
\text { Mitochondrial staining } \\
\text { Western blot } \\
\text { (24-30) } \\
\text { Advanced glycation end } \\
\text { products (40) } \\
\text { RNA sequencing of Malpighian } \\
\text { tubules (18) } \\
\text { Fluid secretion measurement in } \\
\text { Malpighian tubules (30) } \\
\text { Oxidative stress parameters 30) } \\
\text { qRT-PCR ( } n=3 \text { ) }\end{array}$ & 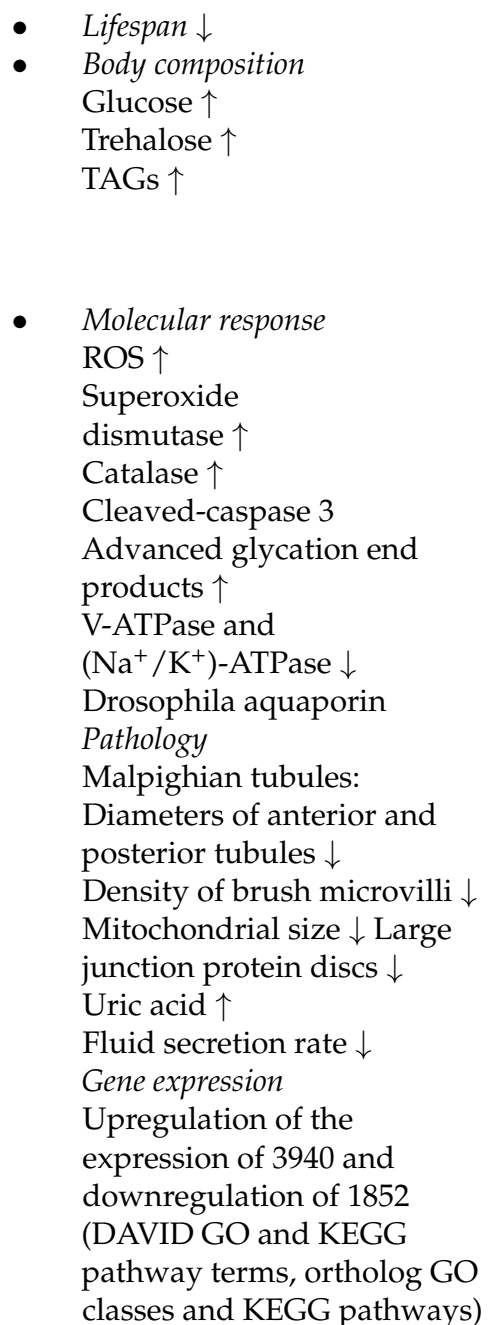 & Rani et al. [140] \\
\hline
\end{tabular}


Table 3. Cont.

\begin{tabular}{|c|c|c|c|c|c|}
\hline Diet & Strain, Sex, Age & Time Period of Intervention & $\begin{array}{c}\text { Assays (Number of Larvae or Flies } \\
\text { per Treatment or } \\
\mathbf{n}=\text { Number of Treatments) }\end{array}$ & Outcomes & Publication \\
\hline $\begin{array}{l}\text { Control diet } \\
5 \% \text { sucrose } \\
10 \% \text { Brewer's yeast } \\
1.5 \% \text { agar } \\
0.3 \% \text { nipagin } \\
0.3 \% \text { propionic acid } \\
\\
\text { HSD } \\
20 \% \text { sucrose } \\
\text { (partly } 30 \%, 40 \% \text { ) }\end{array}$ & $\begin{array}{l}w^{\text {Dah }} ; w^{1118} \\
\text { Males, females } \\
2 \mathrm{~d}\end{array}$ & $\begin{array}{l}7 \text { and } 28 \mathrm{~d} \\
\text { Adjustment of setup: } \\
\text { water source }\end{array}$ & $\begin{array}{ll}\text { - } & \text { Lifespan } \\
& (120-165) \\
& \\
\text { - } \quad \text { Food intake } \\
\text { FlyPAD } \\
\text { CAFE } \\
\text { PER (50) } \\
\text { Con-Ex (50) } \\
\text { Body composition } \\
\text { TAG (32) } \\
\text { Hemolymph } \\
\text { Volume } \\
\text { Glucose } \\
\text { Trehalose } \\
\text { pH (72-96) } \\
\text { Fecundity (150) } \\
\text { - Stress assay } \\
\text { High salt } \\
\text { Oxidative stress } \\
\text { Starvation } \\
\text { (100-200) }\end{array}$ & $\begin{array}{l}\text { - } \quad \text { Lifespan } \\
\text { Dose dependence } \downarrow \text {, rescued } \\
\text { by water supplementation } \\
\text { - } \quad \text { Food intake } \\
\text { FlyPAD } \uparrow \\
\text { CAFE water intake } \uparrow \\
\text { Con-Ex } \uparrow \\
\text { - } \quad \text { Body composition } \\
\text { TAGs } \uparrow \\
\text { Hemolymph } \\
\text { trehalose } \uparrow \\
\\
\text { - } \quad \text { Fecundity } \downarrow \\
\text { Stress tolerance } \\
\text { Salt } \downarrow \\
\text { Starvation } \downarrow \\
\end{array}$ & van Dam et al. [137] \\
\hline
\end{tabular}


Table 3. Cont.

Diet Strain, Sex, Age Time Period of Intervention

Assays (Number of Larvae or Flies

per Treatment or

Outcomes

Publication n = Number of Treatments)

- Molecular analysis

Lipid staining (fat body)

Fat body

Insulin sensitivity

Glucose uptake $(n=5)$

Protein glycation, carbonylation

assay $(n=4-10)$

Intestinal function

Smurf assay (20)

Excreta analysis $(n=5)$

Tubule and rectal ampulla

imaging

Tubule secretion assay

(50)

Metabolomics of rectal ampulla

stones (25 stones)

Uric acid assay (30)

- Molecular response,

pathology

Water imbalance $\uparrow$

Hemolymph volume $\downarrow$

Uric acid $\uparrow$

Glycation adducts $\uparrow$

Insulin resistance

Intestine:

Total number and size of

deposits $\downarrow$

Oblong excreta $\uparrow$

Malpighian tubules: Dark

deposits $\uparrow$

Tubule secretion

rates $\downarrow$

Acidification $\uparrow$

Rectal ampulla stones

(presence and

severity) $\uparrow$

Uric acid in stones $\uparrow$

Dysregulation of purine

biosynthesis 
Table 3. Cont.

\begin{tabular}{|c|c|c|c|c|c|}
\hline Diet & Strain, Sex, Age & Time Period of Intervention & $\begin{array}{c}\text { Assays (Number of Larvae or Flies } \\
\text { per Treatment or } \\
\mathbf{n}=\text { Number of Treatments) }\end{array}$ & Outcomes & Publication \\
\hline $\begin{array}{l}\text { Control diet } \\
5 \% \text { sucrose } \\
6.7 \% \text { yeast } \\
6.7 \% \text { cornmeal } \\
0.5 \% \text { agar } \\
\text { HSD } \\
20 \text { or } 35 \% \text { sucrose }\end{array}$ & $\begin{array}{l}\text { Oregon- } R \\
\text { Males, females } \\
5-6 \mathrm{~d}\end{array}$ & $10 \mathrm{~d}$ & $\begin{array}{ll}\text { - } & \text { Body weight (80) } \\
\text { - } & \text { Locomotion activity } \\
& \text { Climbing assay (150) } \\
\text { Phototaxis assay (150) } \\
\text { - } \quad \text { Body composition } \\
\text { Glucose (60) } \\
\text { - Molecular analysis } \\
\text { Fluorescence microscopy } \\
\text { (20-30 heads, } 20 \text { bodies) } \\
\text { Scanning electron microscopy } \\
\text { (30 heads) } \\
\text { Transmission electron } \\
\text { microscopy (30 heads) }\end{array}$ & $\begin{array}{l}\text { - } \quad \text { Body composition } \\
\text { Glucose } \uparrow \\
\text { - Molecular response } \\
\text { Akt phosphorylation } \uparrow \\
\text { Insulin pathway } \uparrow \\
\text { Retina and lamina: Cleaved } \\
\text { caspase } 3 \uparrow \\
\text { Pathology } \\
\text { Eyes: } \\
\text { Deficit in light } \\
\text { responsiveness } \uparrow \\
\text { Vision defects } \uparrow \\
\text { Ommatidia disorganization } \uparrow \\
\text { Enlarged photoreceptor cell } \\
\text { bodies and rhabdomeres } \\
\text { (volume } \uparrow \text { ) } \\
\text { Apoptosis } \uparrow \\
\text { Eye section: } \\
\text { Autophagy } \uparrow\end{array}$ & Catalani et al. [141] \\
\hline
\end{tabular}


Table 3. Cont.

\begin{tabular}{|c|c|c|c|c|c|}
\hline Diet & Strain, Sex, Age & Time Period of Intervention & $\begin{array}{c}\text { Assays (Number of Larvae or Flies } \\
\text { per Treatment or } \\
\mathbf{n}=\text { Number of Treatments) }\end{array}$ & Outcomes & Publication \\
\hline $\begin{array}{l}\text { Control diet } \\
\text { Yeast } \\
\text { Corn starch } \\
\text { Molasses } \\
\text { HFD } \\
+3,7,15 \text { or } 30 \%(w / v) \\
\text { coconut oil }\end{array}$ & $\begin{array}{l}w^{1118} \\
\text { Females } \\
10-15 \mathrm{~d}\end{array}$ & $\begin{array}{l}\text { Control diet prefeeding } 5 \mathrm{~d} \\
2,5 \text { and } 10 \mathrm{~d} \text { (mainly } 5 \mathrm{~d} \text { ) }\end{array}$ & $\begin{array}{ll}\text { - } & \text { Body composition } \\
\text { TAG } \\
\text { Glucose } \\
(>35) \\
\text { - Locomotion activity } \\
\text { Climbing }(>150) \\
\text { - Molecular analysis } \\
\text { High-resolution } \\
\text { video-microscopy of hearts } \\
(>22) \\
\text { Cardiac TAG }(>90) \\
\text { Gene expression }\end{array}$ & $\begin{array}{ll}\text { - } & \text { Body composition } \\
\text { - } & \text { TAGs } \uparrow \text { (dose-dependent) } \\
\text { Locomotion activity } \\
\text { - } \quad \text { Molimbing } \downarrow \\
\text { pathology response, } \\
\text { Heart: } \\
\text { Pathological alterations } \\
\text { Fat storage } \uparrow \\
\text { Contraction } \\
\text { strength } \downarrow \\
\text { Conduction blocks } \uparrow \\
\text { Dysregulation of insulin-TOR } \\
\text { pathway Changes } \\
\text { insulin/glucose homeostasis }\end{array}$ & Birse et al. [22] \\
\hline $\begin{array}{l}\text { Control diet } \\
5 \% \text { sugar } \\
10 \% \text { yeast } \\
2 \% \text { peptone } \\
1 \% \text { agar } \\
\text { HFD: } \\
14.1 \% \text { "Crisco fat" (soybean } \\
\text { and palm oil) }\end{array}$ & $\begin{array}{l}\text { Canton-S } \\
\text { L3 larvae }\end{array}$ & $\begin{array}{l}\text { Shortterm: } \\
12 \mathrm{~h} \\
\text { or } \\
\text { Longterm: Egg to L3 } \\
\text { Wandering L3 stage }\end{array}$ & $\begin{array}{ll}\text { - } & \text { Food intake } \\
\text { - } & \text { Body size, } \\
\text { Body weight }(>15) \\
\text { Body composition } \\
\text { Hemolymph: } \\
\text { Glucose }(>160) \\
\text { Trehalose }(>15) \\
\text { Whole body: } \\
\text { TAG } \\
\text { Carbohydrates } \\
\text { (>25 larvae, }>30 \text { flies }) \\
\text { Development }(n=3) \\
\text { Molecular analysis } \\
\text { qRT-PCR } \\
\text { Western blot } \\
\text { Lipid droplet measurement } \\
\text { Microarrays }\end{array}$ & $\begin{array}{ll} & \text { Calorie intake } \uparrow \\
\text { - } & \text { Body size } \downarrow \text { body weight } \downarrow \\
\text { - } & \text { Body composition Glucose } \uparrow\end{array}$ & $\begin{array}{l}\text { Palanker Musselman } \\
\text { et al. [129] }\end{array}$ \\
\hline
\end{tabular}


Table 3. Cont.

\begin{tabular}{|c|c|c|c|c|c|}
\hline Diet & Strain, Sex, Age & Time Period of Intervention & $\begin{array}{c}\text { Assays (Number of Larvae or Flies } \\
\text { per Treatment or } \\
\mathbf{n}=\text { Number of Treatments) }\end{array}$ & Outcomes & Publication \\
\hline $\begin{array}{l}\text { Control diet } \\
\text { yeast } \\
\text { corn starch } \\
\text { molasses } \\
\text { HFD } \\
+5,10 \text { or } 20 \%(w / v) \text { coconut } \\
\text { oil (mainly } 20 \%)\end{array}$ & $\begin{array}{l}w^{1118} ; \text { Canton; Oregon- } R \\
\text { Females } \\
3 \mathrm{~d}\end{array}$ & $7 d$ & $\begin{array}{ll}\text { - } & \text { Body composition } \\
\text { TAG }(>85) \\
\text { Glucose }(>60) \\
\text { - } \quad \text { Food intake } \\
\text { CAFE }(70) \\
\text { - } \quad \text { Lifespan }(50) \\
\text { - Stress assay } \\
\text { Anoxia }(>195) \\
\text { Cold }(>210) \\
\text { Starvation }(110)\end{array}$ & $\begin{array}{ll}\text { - } & \text { Body composition } \\
\text { TAGs } \uparrow \\
\text { Glucose } \uparrow \\
\\
\text { - } \quad \text { Lifespan } \downarrow \\
\text { - } \quad \text { Stress tolerance } \\
\text { Cold } \downarrow \\
\text { Anoxic } \downarrow \\
\end{array}$ & Heinrichsen et al. [110] \\
\hline $\begin{array}{l}\text { Control dietyeast } \\
\text { corn starch } \\
\text { molasses } \\
\text { HFD } \\
+30 \%(w / v) \text { coconut oil }\end{array}$ & $\begin{array}{l}w^{1118} \\
\text { Females } \\
5-10 \mathrm{~d}\end{array}$ & $\begin{array}{l}\text { Prefeeding } \\
5 \mathrm{~d} \\
2,5 \text { and } 10 \mathrm{~d} \text { (mainly } 5 \mathrm{~d} \text { ) }\end{array}$ & $\begin{array}{ll}\text { - } & \text { Body composition } \\
\text { TAG }(36) \\
\text { - } \quad \text { Heart analysis } \\
\text { TAG hearts }(>200) \\
\text { Heart periods }(>16) \\
\text { - } \quad \text { Molecular analysis } \\
\text { qRT-PCR }(20) \\
\text { Western blot }(20) \\
\text { Immunocytochemistry (hearts) }\end{array}$ & $\begin{array}{ll}\text { - } & \text { Body composition } \\
\text { TAGs } \uparrow \\
\text { - Heart pathology } \\
\text { Heart: } \\
\text { Lipid droplets } \uparrow \\
\text { Cardiac function } \downarrow \\
\text { Molecular response } \\
\text { Dysregulation PGC- } 1 \text { /spargel } \\
\text { pathway Transcription factor } \\
\text { SREBP } \\
\text { Gene expression } \\
\text { srl } \downarrow\end{array}$ & Diop et al. [23] \\
\hline
\end{tabular}


Table 3. Cont.

\begin{tabular}{|c|c|c|c|c|c|}
\hline Diet & Strain, Sex, Age & Time Period of Intervention & $\begin{array}{c}\text { Assays (Number of Larvae or Flies } \\
\text { per Treatment or } \\
\mathbf{n}=\text { Number of Treatments) }\end{array}$ & Outcomes & Publication \\
\hline $\begin{array}{l}\text { Control diet } \\
10 \% \text { glucose } \\
7 \% \text { corn meal } \\
4 \% \text { yeast } \\
0.6 \% \text { agar } \\
\text { HFD } \\
+5 \% \text { or } 15 \%(w / v) \text { lard }\end{array}$ & $\begin{array}{l}w^{1118} \\
\text { Males, females } \\
\text { L3 larvae } \\
1 \mathrm{~d}\end{array}$ & $\begin{array}{l}\text { L3 larvae } \\
60 \mathrm{~h} \text { post-hatching } \\
10 \mathrm{~d}\end{array}$ & $\begin{array}{ll}\text { - } & \text { Lifespan }(>325) \\
\text { - } & \text { Food intake } \\
& \text { CAFE }(>20) \\
\text { - } & \text { Body composition } \\
& \text { Larvae: Oil red O staining } \\
\text { - } & \text { Molecular analysis } \\
\text { Malondialdehyde measurement } \\
(>40) \\
\text { RT-qPCR (lipid } \\
\text { metabolism-related genes, }>10)\end{array}$ & $\begin{array}{ll}\text { - } & \text { Lifespan } \\
& \text { Median lifespan } \downarrow \\
& \\
\text { - } & \text { Body composition } \\
& \text { Fat content of larval fat } \\
\text { body } \uparrow & \text { Molecular response } \\
\text { - } & \text { Malondialdehyde } \uparrow \\
\text { Gene expression Acox } 57 D-d \downarrow \\
\text { Acsl } \downarrow \\
\text { fabp } \downarrow \\
\text { CCT1 } \downarrow\end{array}$ & Kayashima et al. [8] \\
\hline $\begin{array}{l}\text { Control diet } \\
8 \% \text { fructose } \\
10 \% \text { yeast } \\
2 \% \text { polenta } \\
0.8 \% \text { agar } \\
\text { HFD } \\
+6.3 \text { or } 15 \%(w / v) \text { lard }\end{array}$ & $\begin{array}{l}w^{1118} \\
\text { Males }\end{array}$ & $10,20,30,40 \mathrm{~d}$ & $\begin{array}{ll}\text { - } & \text { Lifespan }(>170) \\
\text { - } & \text { Food intake } \\
\text { Feeding assay }(>15) \\
\text { Body composition } \\
\text { Glucose } \\
\text { Trehalose } \\
\text { - } 12) \\
\text { Molecular analysis } \\
\text { Insulin sensitivity }(9) \\
\text { Thin-layer chromatography }(40) \\
\text { qRT-PCR } \\
\text { Confocal microscopy }(>10) \\
\text { Bacterial infection }(4-5) \\
\text { Fluorescence-activated cell } \\
\text { sorting (90) } \\
\text { Oil Red O staining of } \\
\text { FACS-sorted plasmatocytes } \\
\text { (>35 cells) } \\
\text { Plasmatocyte gene expression } \\
\text { arrays }(>5000 \text { plasmatocytes) } \\
\text { Plasmatocyte cell death }\end{array}$ & $\begin{array}{ll}\text { - } & \text { Lifespan } \downarrow \\
& \\
\text { - } & \text { Body composition } \\
\text { TAGs } \uparrow \\
\text { Glucose } \uparrow \\
\text { Trehalose } \uparrow \\
\text { - Molecular response, pathology } \\
\text { Insulin sensitivity } \uparrow \\
\text { Muscle, midgut: } \\
\text { 10xSTAT92E-GFP } \uparrow \\
\text { Gene expression } \\
\text { Whole genome: } \\
\text { ap- } 1 \uparrow \\
\text { Fox } \uparrow \\
\text { ATF-CREB binding } \\
\text { sites } \uparrow \\
\text { upd } \uparrow\end{array}$ & Woodcock et al. [124] \\
\hline
\end{tabular}


Table 3. Cont.

\begin{tabular}{|c|c|c|c|c|c|}
\hline Diet & Strain, Sex, Age & Time Period of Intervention & $\begin{array}{c}\text { Assays (Number of Larvae or Flies } \\
\text { per Treatment or } \\
\text { n = Number of Treatments) }\end{array}$ & Outcomes & Publication \\
\hline $\begin{array}{l}\text { Control diet } \\
10 \% \text { sugar } \\
10 \% \text { yeast } \\
1.5 \% \text { agar } \\
1 \% \text { Tween } 80(w / v) \\
\text { HFD } \\
+2 \%(w / v) \text { palmitic acid }\end{array}$ & $\begin{array}{l}\text { Canton-S } \\
\text { Males } \\
3 \mathrm{~d}\end{array}$ & $\begin{array}{l}\text { Prefeeding } \\
3 \mathrm{~d} \\
7 \text { and } 14 \mathrm{~d}\end{array}$ & $\begin{array}{ll}-\quad & \text { Lifespan }(100) \\
- & \text { Locomotion activity } \\
& \text { Climbing (200) } \\
\text { - } & \text { Sensory perception } \\
& \text { Odor stimulation }(15) \\
& \text { T-maze }(200) \\
& \text { Electrophysiological } \\
& \text { recordings (15) } \\
\text { - } & \text { Molecular analysis } \\
& \text { q-RT-PCR } \\
& \text { Gene expression }(n=3)\end{array}$ & $\begin{array}{ll}\text { - } & \text { Lifespan } \downarrow \\
& \text { Locomotion activity } \\
\text { - } & \text { Climbing } \downarrow \\
& \text { Olfactory perception } \\
& 10 \text { aromatic compounds } \downarrow \\
\text { Changed reaction } \\
\text { to aromatics } \\
\text { Molecular response Changes in } \\
\text { olfactory and nutrient-related } \\
\text { signaling pathways } \\
\text { Gene expression } \\
\text { Antennas: DmOrco } \downarrow\end{array}$ & Jung et al. [146] \\
\hline $\begin{array}{l}\text { Control diet } \\
0.75 \% \text { sucrose } \\
1.3 \% \text { yeast } \\
6.5 \% \text { cornmeal } \\
0.7 \% \text { agar } \\
\text { HFD } \\
+3,7,15 \text { or } 30 \%(w / v) \\
\text { coconut oil }(\text { mainly } 15 \%)\end{array}$ & $\begin{array}{l}\text { Canton-S } \\
\text { Females } \\
\text { Post eclosion }\end{array}$ & $\begin{array}{l}\text { During development or only } \\
\text { in adults }\end{array}$ & $\begin{array}{ll}\text { - } & \text { Body length } \\
& \text { (3\% HFD; 100) } \\
\text { - } \quad \text { Locomotion activity } \\
\text { - } & \text { Courivity level } \\
\text { Courtship } \\
\text { Mating success } \\
\text { Courtship latency } \\
\text { Courtship index } \\
\text { Mating latency } \\
\text { Competition (25) } \\
\text { - Courtship song recording (10) } \\
\text { Development (3\% HFD) } \\
\text { Molecular analysis } \\
\text { Cuticular hydrocarbon }(40) \\
\text { GC/MS analysis (40) } \\
\text { Metabolic rescue (30) }\end{array}$ & 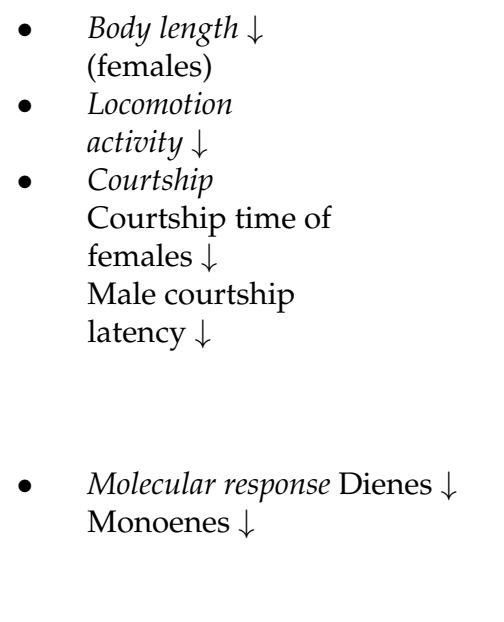 & Schultzhaus et al. [149] \\
\hline
\end{tabular}


Table 3. Cont.

\begin{tabular}{|c|c|c|c|c|c|}
\hline Diet & Strain, Sex, Age & Time Period of Intervention & $\begin{array}{c}\text { Assays (Number of Larvae or Flies } \\
\text { per Treatment or } \\
\text { n = Number of Treatments) }\end{array}$ & Outcomes & Publication \\
\hline $\begin{array}{l}\text { Control diet } \\
10 \% \text { sugar } \\
10 \% \text { yeast } \\
2 \% \text { agar } \\
\text { HFD } \\
+30 \%(w / v) \text { coconut oil }\end{array}$ & $\begin{array}{l}w^{1118} \\
\text { Females } \\
2 \mathrm{~d}\end{array}$ & 5 weeks & $\begin{array}{ll} & \text { Lifespan }(>200) \\
\text { - } & \text { Locomotion activity } \\
& \text { Climbing }(>100) \\
\text { - } & \text { Body composition } \\
& \text { TAG }(40) \\
\text { - } & \text { Heart analysis } \\
& \text { Heart contraction (30) } \\
& \\
\text { - } & \text { Molecular analysis qRT-PCR (10) }\end{array}$ & $\begin{array}{ll}\text { - } & \text { Lifespan } \downarrow \\
\text { - } & \text { Locomotion activity } \\
\text { - } & \text { Climbing } \downarrow \\
& \text { Body composition Whole-body } \\
& \text { TAGs } \uparrow \\
\text { - } & \text { Heart analysis } \\
& \text { Cardiac TAGs } \uparrow \\
\text { Heart rate } \uparrow \\
\text { Fibrillation } \uparrow \\
\text { Fractional shortening } \downarrow \\
\text { - } \quad \text { Gene expression } \\
\quad d \text { Sir } 2 \downarrow\end{array}$ & Wen et al. [148] \\
\hline $\begin{array}{l}\text { Control diet } \\
8 \% \text { sucrose } \\
0.5 \% \text { yeast } \\
2 \% \text { agar } \\
0.16 \% \text { calcium chloride } \\
\text { dihydrate } \\
0.16 \% \text { ferrous sulfate } \\
\text { heptahydrate } \\
0.8 \% \text { sodium potassium } \\
\text { tartrate tetrahydrate } \\
0.05 \% \text { sodium chloride } \\
0.05 \% \text { manganese chloride } \\
\text { tetrahydrate } \\
0.53 \% \text { nipagin } \\
\text { HFD } \\
+5 \%(w / v) \text { coconut oil }\end{array}$ & $\begin{array}{l}\text { Canton-S; } w^{1118} \\
\text { Males, females } \\
\text { 1, 3, } 5 \text { weeks }\end{array}$ & $4 \mathrm{~d}$ & $\begin{array}{ll}\text { - } & \text { Body weight } \\
\text { Body composition } \\
\text { TAG } \\
(>30) \\
\text { Hemolymph: } \\
\text { Glucose } \\
\text { Trehalose } \\
(>100) \\
\text { - Food intake } \\
\text { CAFE }(>50) \\
\text { - Starvation }(50) \\
\text { - Lifespan }(100) \\
\text { Locomotion activity }(50) \\
\text { Flight }(>100) \\
\text { Climbing }(>100)\end{array}$ & $\begin{array}{l}\text { - } \quad \text { Body composition } \\
\text { TAGs } \uparrow \\
\text { Hemolymph } \\
\text { Glucose } \uparrow \\
\text { Trehalose } \uparrow \\
\\
\\
\text { - } \quad \text { Lifespan } \downarrow \\
\text { Locomotion activity } \\
\text { Climbing ability } \downarrow \text { Flight } \\
\text { performance } \downarrow\end{array}$ & Villanueva et al. [134] \\
\hline
\end{tabular}


Table 3. Cont.

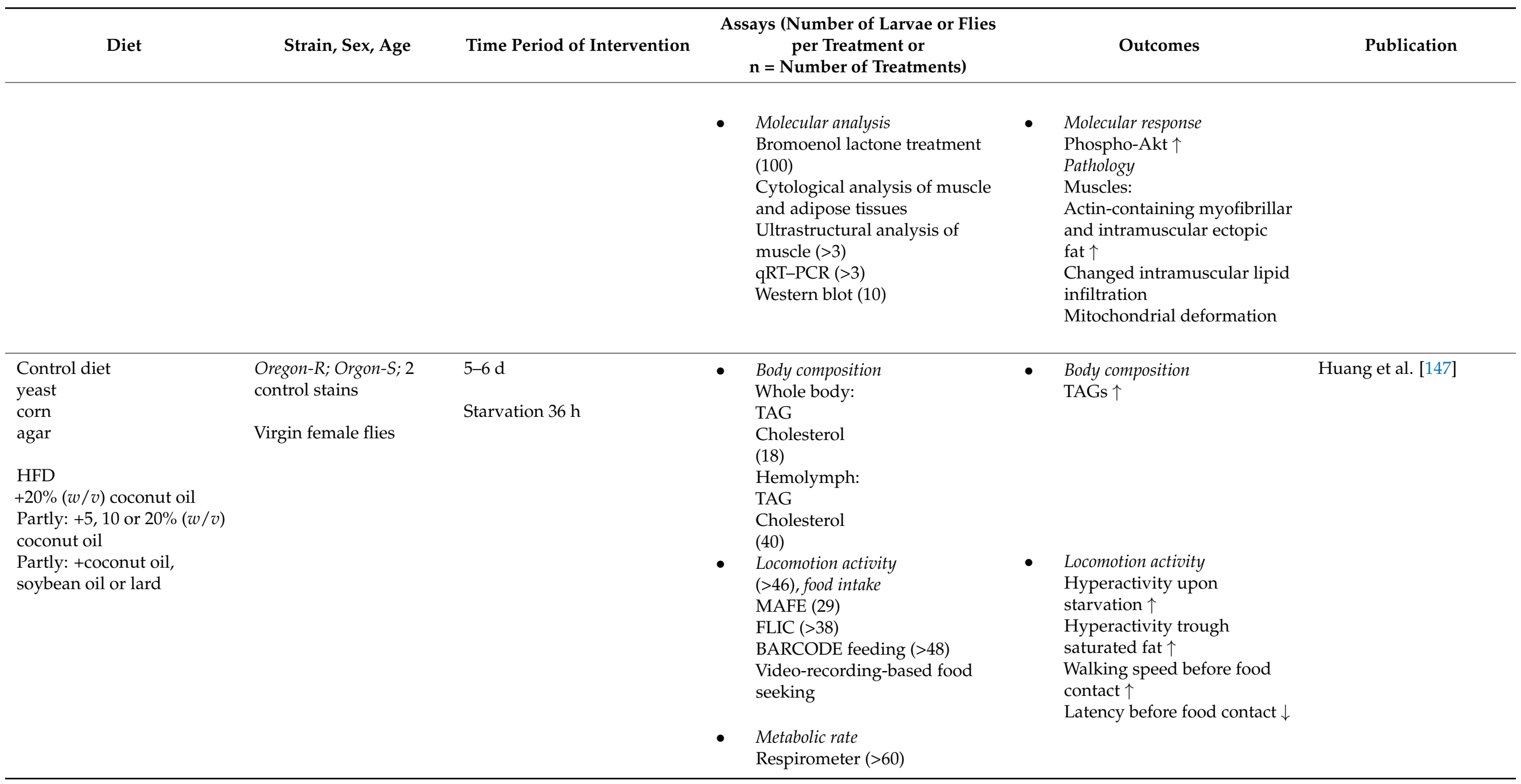


Table 3. Cont.

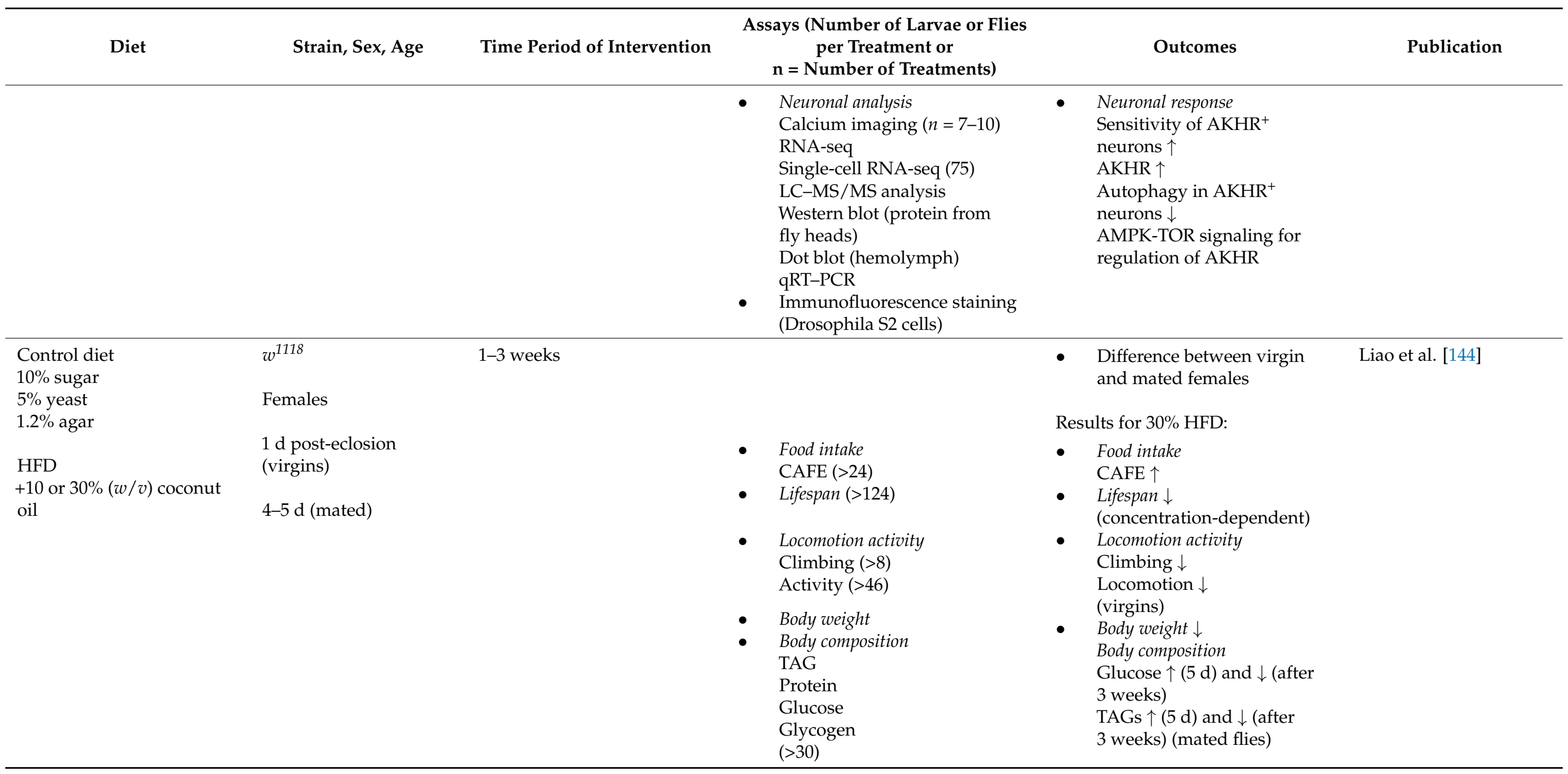


Table 3. Cont.

\begin{tabular}{|c|c|c|c|c|c|}
\hline Diet & Strain, Sex, Age & Time Period of Intervention & $\begin{array}{c}\text { Assays (Number of Larvae or Flies } \\
\text { per Treatment or } \\
\mathbf{n}=\text { Number of Treatments) }\end{array}$ & Outcomes & Publication \\
\hline & & & $\begin{array}{ll}\text { - } & \text { Fecundity }(>19) \\
\text { - } & \text { Cold stress assay }(>80) \\
\text { - } & \text { Molecular analysis } \\
& \text { Immunostaining (brain, crops, } \\
& \text { heart, muscle, }>8 \text { ) } \\
\text { qRT-PCR }\end{array}$ & $\begin{array}{ll}\text { - } & \text { Fecundity } \downarrow \\
\text { - } & \text { Stress tolerance } \\
& \text { Faster recovery (cold) } \\
\text { - } & \text { Molecular response } \\
& \text { Akh } \uparrow \text { (virgins) } \\
& \text { Pathology } \\
\text { Crop size } \uparrow\end{array}$ & \\
\hline
\end{tabular}


Furthermore, an HFD adversely affects stress resistance, e.g., resistance to exposure to extreme cold and anoxia [110]. The increased stress susceptibility and reduced health status lead to detrimental impacts on locomotor activity, flight ability and survivorship $[8,110,134,144,148]$.

HFD-induced alterations in tissues can be observed. HFD consumption leads to the upregulation of phospho-protein kinase B (Akt) levels, which causes the inhibition of the transcription factor, Forkhead Box O $(d F O X O)$. The downregulation of nuclear gene expression and increased activity of the Drosophila target of rapamycin (dTOR) follows, which in turn inhibits spargel and activates FA synthases (FASs). These metabolic adaptations ultimately promote fat accumulation, lipotoxic heart dysfunction and change skeletal muscle physiology (e.g., causing the disorganization of actin-containing myofibrils) in D. melanogaster $[22,23,134]$.

Moreover, it was shown in many studies that the reproductive capacity (e.g., fecundity) of females is decreased [144], which is affected by the deterioration of activity and flight capacity and changes in the visual (body size) and nonvisual (pheromones) sex characteristics of female flies [149].

\section{Molecular Changes Following a High-Sugar Diet and High-Fat Diet}

D. melanogaster phenotyping in response to dietary factors such as HSD and HFD can be complemented with transcriptomic and metabolomic analyses to unravel the underlying molecular mechanisms, as indicated below.

\subsection{Changes in Transcriptome}

In recent years, several studies were published that evaluated the relation between the intake of either an HSD or HFD and gene expression patterns in D. melanogaster. An overview summarizing the different dietary regiments and the main outcomes including the number and examples of up- and downregulated genes is given in Table 4.

\subsubsection{Transcriptional Changes Induced by HSD}

HSD consumption considerably affects the development of fruit flies, especially at larval stages. Palanker Musselman et al. (2011) analyzed whole-body gene expression in male Canton-S larvae, aiming to identify differences in gene expression patterns following the ingestion of either a control diet or an HSD containing $1 \mathrm{M}$ (equivalent to $34 \%$ ) sucrose for a short-term (for $12 \mathrm{~h}$, starting at mid-L3 stage) or long-term (egg to wandering L3 stage) period. Both short-term and long-term HSD intake increased the mRNA levels of genes involved in glucose transport, FA and trehalose synthesis and TAG storage. Shortterm HSD ingestion increased the transcription of genes that regulate glycogenolysis and the pentose phosphate shunt and reduced the expression of genes involved in glycolysis. Long-term HSD feeding elevated the expression of genes that regulate gluconeogenesis and $\beta$-oxidation. In this regard, marked alterations in the transcription of $d F O X O$ downstream target genes were evident. These findings indicate causes for the development of an insulin resistance phenotype in L3 larvae following HSD treatment [129].

In contrast, a short-term feeding $(24 \mathrm{~h})$ with an HSD containing only $16 \%$ sucrose did not alter gene expression patterns of L3 larvae from two iso female wild-type strains (Iso-SD1 and Iso-SD3). The authors conclude that D. melanogaster are fruit breeders and adapt much better to sugar-rich nutrition than other Drosophila species (e.g., D. arizonae, D. mojavensis) that are cactus breeders; therefore, no changes at the transcriptional level are observed following HSD consumption [150].

Starting from eggs, Loreto et al. (2021) reared Oregon- $R$ flies on a $30 \%$ sucrose HSD and $2.5 \%$ sucrose control medium, respectively. However, in this study, whole-body gene expression was not investigated in larvae but in 7-day old adults. HSD-fed D. melanogaster exhibit an up-regulation of genes involved in ribosomal biogenesis and a down-regulation of genes involved in energy metabolism, including glycolysis, TCA cycle and ATP synthesis, as well as genes involved in insect development. Together with the downregulated 
expression of Actin (Act88F) these changes in gene expression may result in the disruption of muscle development and mitochondrial dysfunction, which is phenotypically evident in developmental delay and muscle atrophy. In addition, the expression profile of flies fed with $30 \%$ HSD, in terms of the insulin-like pathway, indicates that flies may develop type 2 diabetes mellitus-like phenotype [151].

Hemphill et al. (2018) fed an HSD (20\% sucrose) for 7 days to Oregon $R-C$ females and examined gene expression in the fly heads. In good accordance with the increased energy intake, genes that function in peptide and carbohydrate metabolism (protein cleavage, enzyme activation, and macromolecule processing) were found to be enriched, predominately including genes involved in sugar/carbohydrate processing. Furthermore, genes relating to checkpoint kinases were upregulated by the HSD, which together with the decreased expression of genes related to the cell cycle, was discussed in the context of high-dietary-sugar-induced DNA damage. The upregulation of the transcription of heat shock proteins, genes involved in antimicrobial defense and genes of apoptotic processes, was also evident, indicating that HSD-fed animals experienced stress. In good accordance with the increased TAG storage, $A k h$ is downregulated and heimdall is upregulated by the HSD [152].

As described by $\mathrm{Ng}^{\prime}$ oma et al. (2020), 10 days of feeding of an HSD containing $1 \mathrm{M}$ sucrose alters multiple genetic networks (examined with RNA-seq) in outbred females (Drosophila Synthetic Population Resource) in comparison to control-fed flies. Although genes encoding the proteins of insulin/insulin-like signaling/the TOR pathway, including Dilp5, Rheb, and Sirt2, showed a significant elevation in expression, the effect of an HSD was not limited to key genes but rather affected a wide range of biological pathways and cofactors [153].

An HSD that is solely administered in early adult life affects long-term health outcomes at older ages. The reversible inhibition of the activity of the transcription factor dFOXO, after a week-long treatment with an HSD containing 40\% sucrose, was indicated by RNA-seq analyses. The inhibition of $A F O X O$ alters the downstream gene transcription of chromatin/nucleosome remodelers and histone-modifying enzymes in wild-type female flies. Moreover, the inhibition of dFOXO leads to reprogramming at the transcriptome level, which affects lifespan [136]. This summary of transcriptome studies indicates that different dietary treatment regiments (varying sugar contents of the HSD and periods of intervention) targeting different sexes, wild-type strains and stages of the fly life cycle (larvae feeding only, larvae to adult feeding, adult feeding only) were applied to examine the impact of HSD on gene expression. In addition, gene expression was analyzed in different parts of the fly body (whole body, heads only). Although this heterogeneity hampers data comparison, we tried to extract general trends in HSD-induced gene expression, which are summarized in Figure 12.

\subsubsection{Transcriptional Changes Induced by HFD}

Ruden and co-workers [154,155] presented the combination of high dimensional biological data with complex diseases and quantitative traits and gave guidelines for the design, analysis and interpretation of corresponding experiments. They further released important work in the field of nutrigenomics (as the study of gene-nutrient interaction) by using Drosophila "whole genome" arrays in response to various dietary conditions.

In all studies that will be presented below, the HFD comprises $20 \%(w / v)$ coconut oil and was fed for 7 days to adult fruit flies.

The impact of consumption of an HFD on the transcriptome was investigated in female $w^{1118}$ D. melanogaster by Heinrichsen et al. (2014). In particular, the immune response, carbohydrate binding, iron transport and extracellular secretion are modified by the altered mRNA levels. In addition, the expression levels of genes that are associated with the metabolism of amino acids and glycerophospholipids, as well as the degradation of sugars and waste metabolites, are changed. Another factor is the diminishment of CG9510 expression (the homolog of mammalian $A S L$ ), which is involved in carbon and nitrogen 
metabolism. Downregulation of CG9510 expression unfavorably increases fat storage, impairs cardiac function, decreases tolerance to stress and shortens lifespan [156].

\begin{tabular}{|c|c|}
\hline Upregulated transcription & Downregulated transcription \\
\hline $\begin{array}{l}\text { Metabolism \& energy provision } \\
\text { - Sugar molecule processing } \\
\text { - Insulin signaling pathway } \\
\text { - Mitochondrial activity } \\
\text { - Metabolic enzymes } \\
\text { - TAG storage } \\
\text { - Proteolysis } \\
\text { - Peptide processing } \\
\text { Infection \& stress response } \\
\text { - Heat shock proteins } \\
\text { - Cytochrome P450 } \\
\text { - HK kinase-like proteins } \\
\text { - Ribosomal biogenesis } \\
\text { Nuclear regulations } \\
\text { - Signal transduction }\end{array}$ & $\begin{array}{l}\text { Metabolism \& energy provision } \\
\text { - Pyruvate generation } \\
\text { - TCA cycle } \\
\text { - ATP synthesis } \\
\text { - Kinases } \\
\text { - } \quad \text { dFOXO } \\
\text { Development } \\
\text { - Muscle development } \\
\text { Infection \& stress response } \\
\text { - Cytochrome P450 } \\
\text { Nuclear regulations } \\
\text { - DNA binding \& transcription } \\
\text { - Cell cycle }\end{array}$ \\
\hline
\end{tabular}

Figure 12. Transcriptional alterations following intake of a high-sugar diet (HSD) in Drosophila melanogaster. Ingestion of an HSD causes upregulation (blue box) and downregulation (red box) of genes involved in metabolism and energy provision, infection and stress responses, nuclear regulation, and development (modified from [129,136,151-153]).

Ingestion of an HFD in adult $w^{1118}$ female D. melanogaster affected genes expressed in heads encoding odorant/pheromone proteins, cytochrome P450, monooxygenase, and enzymes that account for antioxidant capacity [157]. The increased transcription of odorant binding proteins may be associated with efforts to search for other nutrient sources. Genes that encode enzymes that are related to nucleotide metabolism are also transcribed to a greater extent in the head of HFD-fed flies than in controls. HFD mainly downregulates the expression of genes that encode factors that are involved in DNA replication, mitosis, cell cycle regulation, RNA binding and neurogenesis. Downregulation of the neuronal expression of these genes was discussed in the context of a higher response to oxidative stress, neuronal substrate exhaustion and HFD-induced mild neuronal deterioration and impairment of short- and long-term memory as well as disturbance of neurophysiological mechanisms (e.g., neuromuscular signaling) and locomotion. In addition, ingestion of an HFD is associated with a reduction in the expression of doppelganger of brummer (dob), which is related to an increase in fat storage [157].

However, the previously reported impact of the expression of $d o b$ on fat accumulation cannot be validated, in that a lack of dob activity in adult $D$. melanogaster mutants has not been found to modify the TAG content. It can be inferred that dob exhibits bmm activity in the fat body but remains nonessential for the regulation of chronic TAG storage [158].

Stobdan et al. (2019) reported that feeding with an HFD induces shifts in transcription patterns in adult $w^{1118} D$. melanogaster compared to the respective controls that are sex and organ-specific (measured via RNA-seq of heads and bodies) [159]. Males are affected to a greater extent than females, as the transcription of a higher number of genes is altered in males (Table 4). In males, downregulation of whole-body glycoside hydrolases (e.g., lysosomal $\alpha$-mannosidase (LManIII-VI)), which are significantly involved in carbohydrate 
metabolism, at the transcriptional level is evident. The expression of these genes may be related to sperm quality and function in D. melanogaster [160]. In addition, the expression of genes encoding proteins that are associated with stress and the immune response is altered in the heads of males. This may alter the stress tolerance of males following HFD consumption [161]. In females, alteration at the transcriptional level is present for genes involved in lipid metabolism. Ingestion of an HFD induces transcription of numerous related genes in the heads of flies. In males and females, the transcription of Cytochrome $\mathrm{P} 4504 \mathrm{e} 3$ (Cyp4e3), which is associated with antioxidant capacity, and takeout (to), which is associated with hyperphagic behavior of the fruit fly, is upregulated. In the fat body, the changes in the expression profile likely contribute to the regulation of lipid homeostasis in both males and females [159].

Results published by Azuma et al. (2019) validate the abovementioned findings, as the researchers found that consumption of an HFD similarly modified whole-body gene expression (determined by RNA-seq) in adult Canton-S D. melanogaster. Genes involved in the regulation of the energy metabolism, fatty acid synthesis and modification as well as in carbohydrate metabolism were affected. Accordingly, changes in the transcript frequency of these genes were associated with alterations in glucose metabolism and gluconeogenesis. Again, the transcription of genes that encode key factors in the oxidative stress response was increased, while genes encoding antibacterial proteins was diminished, leading to impairment of immune system function. In females, the expression of genes that are critically involved in egg production, was decreased, impairing oogenesis [162].

Hemphill et al. (2018) examined the HFD-induced changes in gene expression in the heads of Oregon $R-C$ females. Similar to HSD investigated in the same study, ingestion of the HFD upregulates the expression of genes encoding proteins that are involved in peptide and carbohydrate metabolism. Upregulation of transcription of heat shock proteins, indicating induction of the stress response, and of genes of antimicrobial peptides and immune function and the apoptotic processes was also evident. On the other hand, intake of an obesogenic diet leads to downregulation, e.g., of the expression of heimdall (CG4500), which affects TAG storage and disturbs sleep homeostasis [152]. In addition, the transcription of genes related to chitin metabolic processing and metabolic functions is increased, which may promote circulation of excess metabolites. Nutrient storage, signal transmission and pyridoxal phosphate function are downregulated at the transcriptional level by an HFD.

Compared to the HSD studies presented above, the studies that examined the changes in the transcriptome of fruit flies in response to an HFD are by far more homogeneous in terms of the dietary regiment. In most cases, $20 \%$ coconut oil was fed for 7 days to adult flies. Accordingly, the data lead to a more coherent picture of the HFD induced transcriptional changes that summarized in Figure 13. On the other hand, several open questions regarding, e.g., the effects of fat quality and fat quantity on differential gene expression remain to be elucidated. 
Table 4. Overview of dietary intervention studies regarding changes in transcriptome following high-sugar diet (HSD) or high-fat diet (HFD) feeding in Drosophila melanogaster. The studies are ordered by release date.

\begin{tabular}{|c|c|c|c|c|c|}
\hline Diet & Strain, Sex, Age & Time Period of Intervention & $\begin{array}{l}\text { Method } \\
\text { (Tissue) }\end{array}$ & $\begin{array}{l}\text { Outcomes } \\
\text { (Process, e.g., Genes) }\end{array}$ & Publication \\
\hline \multicolumn{6}{|l|}{ HSD } \\
\hline $\begin{array}{l}\text { Control diet } \\
5 \% \text { sugar } \\
10 \% \text { yeast } \\
2 \% \text { peptone } \\
1 \% \text { agar } \\
\\
\text { HSD } \\
34 \% \text { sugar }\end{array}$ & $\begin{array}{l}\text { Canton-S } \\
\text { Males } \\
\text { L3 larvae }\end{array}$ & $\begin{array}{l}\text { Shortterm: } \\
12 \mathrm{~h} \\
\text { or } \\
\text { Longterm: Egg to L3 } \\
\text { Wandering L3 stage }\end{array}$ & $\begin{array}{l}\text { Microarray } \\
\text { (whole body) }\end{array}$ & $\begin{array}{l}\text { Results short-term intervention: } \\
\text { - } \quad \text { Genes altered } \\
\text { 823 genes } \\
\text { Genes upregulated } \\
\text { Glucose transport (Uggt) } \\
\text { Fatty acid (FA) synthesis } \\
\text { (FASN) } \\
\text { Trehalose synthesis (Treh) } \\
\text { Triacylglyceride (TAG) storage } \\
\text { (TAG lipases) } \\
\text { Glycogenolysis (GlyP) Pentose } \\
\text { phosphate shunt (G6PD) } \\
\text { Genes downregulated } \\
\text { Glycolysis (Hex) } \\
\text { Results long-term intervention: } \\
\text { Genes altered } \\
\text { 309 genes } \\
\text { Genes upregulated } \\
\text { Glucose transport (Uggt) } \\
\text { FA synthesis (FASN) } \\
\text { Trehalose synthesis (Treh) } \\
\text { TAG storage (TAG lipases) } \\
\text { Gluconeogenesis (Pepck) } \\
\beta \text {-oxidation (Acetyl-coenzyme } \\
\text { A (acyl-CoA) dehydrogenases) } \\
\text { Insulin signaling pathway } \\
\text { (dFOXO) }\end{array}$ & $\begin{array}{l}\text { Palanker Musselman et al. } \\
\text { [129] }\end{array}$ \\
\hline
\end{tabular}


Table 4. Cont.

\begin{tabular}{|c|c|c|c|c|c|}
\hline Diet & Strain, Sex, Age & Time Period of Intervention & $\begin{array}{l}\text { Method } \\
\text { (Tissue) }\end{array}$ & $\begin{array}{l}\text { Outcomes } \\
\text { (Process, e.g., Genes) }\end{array}$ & Publication \\
\hline $\begin{array}{l}\text { Control diet } \\
5 \% \text { sucrose } \\
10 \% \text { yeast } \\
1.5 \% \text { agar } \\
\text { HSD } \\
40 \% \text { sucrose }\end{array}$ & $\begin{array}{l}w^{1118} \\
\text { Females } \\
2 \mathrm{~d}\end{array}$ & $\begin{array}{l}1-3 \text { weeks, followed by } 1 \\
\text { week on control diet }\end{array}$ & $\begin{array}{l}\text { RNA sequencing } \\
\text { (whole body) }\end{array}$ & $\begin{array}{ll}-\quad \text { Genes altered } \\
\text { - } \quad \text { Genes upregulated } \\
\text { Insulin signaling pathway } \\
\text { (Dilp6) } \\
\text { - Genes downregulated } \\
\text { Insulin signaling pathway } \\
\text { (dFOXO) } \\
\text { Histone-modifying enzymes } \\
(\text { Acf, D12, egg) }\end{array}$ & Dobson et al. [136] \\
\hline $\begin{array}{l}\text { Control diet } \\
3 \% \text { sucrose } \\
4 \% \text { inactive yeast } \\
2.6 \% \text { cornmea } \\
10.8 \% \text { agar } \\
1.5 \% \text { tegosept } \\
0.3 \% \text { propionic acid } \\
\text { HSD } \\
20 \% \text { sucrose }\end{array}$ & $\begin{array}{l}\text { Oregon } R-C \\
\text { Females } \\
2 \mathrm{~d}\end{array}$ & $7 \mathrm{~d}$ & RNA sequencing (heads) & $\begin{array}{l}\text { - Genes upregulated } \\
164 \text { genes } \\
\text { Peptide metabolism } \\
\text { Carbohydrate metabolism } \\
\text { Hexokinase-like proteins } \\
\text { Cytochrome P450 } \\
\text { Mitochondrial activity } \\
\text { Stress response }(h s p) \\
\text { Infection defense, antimicrobial } \\
\text { peptide production }(D p t) \\
\text { Apoptotic processes }(\text { Decay) } \\
\text { Genes downregulated } \\
69 \text { genes } \\
\text { DNA binding } \\
\text { Transcription } \\
\text { Cytochrome P450 function } \\
\text { Kinase activity } \\
\text { heimdall (CG4500) }\end{array}$ & Hemphill et al. [152] \\
\hline
\end{tabular}


Table 4. Cont.

\begin{tabular}{|c|c|c|c|c|c|}
\hline Diet & Strain, Sex, Age & Time Period of Intervention & $\begin{array}{l}\text { Method } \\
\text { (Tissue) }\end{array}$ & $\begin{array}{l}\text { Outcomes } \\
\text { (Process, e.g., Genes) }\end{array}$ & Publication \\
\hline $\begin{array}{l}\text { Control diet } \\
2 \% \text { sucrose } \\
22 \% \text { cornmea } \\
13 \% \text { wheat germ } \\
0.1 \% \text { powdered milk } \\
0.1 \% \text { salt } \\
0.1 \% \text { soybean flour } \\
0.1 \% \text { rye flour } \\
\text { Nipagin } \\
\text { Lyophilized yeast } \\
\text { HSD } \\
30 \% \text { sucrose }\end{array}$ & $\begin{array}{l}\text { Oregon- } R \\
\text { Males, females } \\
\text { Eggs on control diet/HSD }\end{array}$ & $7 \mathrm{~d}$ & $\begin{array}{l}\text { RNA sequencing } \\
\text { (whole body) }\end{array}$ & $\begin{array}{l}\text { - Genes upregulated } \\
2695 \text { genes } \\
\text { Nuclear regulation processes } \\
\text { Ribosome biogenesis target of } \\
\text { rapamycin } \\
\text { Mitogen-activated } \\
\text { protein kinase } \\
\text { Extracellular signal-regulated } \\
\text { kinases (ERKs) } \\
\text { Insulin signaling (Dilps7, 8) } \\
\text { Genes activated from zero: } \\
\text { 3\% } \\
\text { Muscle structure } \\
\text { Developmental growth } \\
\text { Tissue development } \\
\text { Genes downregulated } \\
1136 \text { genes } \\
\text { Development muscle cells } \\
\text { (Actin) } \\
\text { Generation of precursor } \\
\text { metabolites, enzymes and } \\
\text { components of key energy } \\
\text { pathways (Pyk, } k d n, A T P s y n C) \\
\text { Insulin signaling pathway } \\
\text { (Dilp6, p53 (isoform } A \text { )) } \\
\text { Genes knocked down: } \\
4 \% \\
\text { Cell structure } \\
\text { Development }\end{array}$ & Loreto et al. [151] \\
\hline
\end{tabular}


Table 4. Cont.

\begin{tabular}{|c|c|c|c|c|c|}
\hline Diet & Strain, Sex, Age & Time Period of Intervention & $\begin{array}{l}\text { Method } \\
\text { (Tissue) }\end{array}$ & $\begin{array}{l}\text { Outcomes } \\
\text { (Process, e.g., Genes) }\end{array}$ & Publication \\
\hline $\begin{array}{l}\text { Control diet } \\
\text { yeast } \\
\text { corn starch } \\
\text { molasses } \\
\text { HFD } \\
20 \%(w / v) \text { coconut oil }\end{array}$ & $\begin{array}{l}w^{1118} \\
\text { Females } \\
3-5 \mathrm{~d}\end{array}$ & $7 \mathrm{~d}$ & $\begin{array}{l}\text { Microarray } \\
\text { (whole body) }\end{array}$ & $\begin{array}{l}\text { - Genes upregulated } \\
1030 \text { genes } \\
\text { Immune response } \\
\text { Carbohydrate binding } \\
\text { Iron transport } \\
\text { Extracellular secretion } \\
\text { Amino acid metabolism } \\
\text { Glycerophospholipids } \\
\text { metabolism } \\
\text { Sugar degradation } \\
\text { Waste metabolites degradation } \\
\text { Genes downregulated } \\
297 \text { genes } \\
\text { CG9510 }\end{array}$ & Heinrichsen et al. [156] \\
\hline $\begin{array}{l}\text { Control diet } \\
3 \% \text { sucrose } \\
4 \% \text { inactive yeast } \\
2.6 \% \text { cornmea } \\
10.8 \% \text { agar } \\
1.5 \% \text { tegosept } \\
0.3 \% \text { propionic acid } \\
\text { HFD } \\
20 \%(w / v) \text { coconut oil }\end{array}$ & $\begin{array}{l}\text { Oregon } R-C \\
\text { Females } \\
2 \mathrm{~d}\end{array}$ & $7 \mathrm{~d}$ & RNA sequencing (heads) & $\begin{array}{l}\text { Genes upregulated } \\
197 \text { genes } \\
\text { Peptide metabolism } \\
\text { Carbohydrate metabolism } \\
\text { Chitin metabolic processing } \\
\text { Metabolic functions } \\
\text { Stress response }(h s p) \\
\text { Infection defense, immune } \\
\text { function (CecA1, AttA) } \\
\text { Antimicrobial peptide } \\
\text { production }(D p t) \\
\text { Apoptotic processes } \\
\text { (Decay) } \\
\text { Genes downregulated } \\
\text { 36 genes } \\
\text { Nutrient storage } \\
\text { Signal transmission } \\
\text { Pyridoxal phosphate function } \\
\text { heimdall (CG4500) }\end{array}$ & Hemphill et al. [152] \\
\hline
\end{tabular}


Table 4. Cont.

\begin{tabular}{|c|c|c|c|c|c|}
\hline Diet & Strain, Sex, Age & Time Period of Intervention & $\begin{array}{l}\text { Method } \\
\text { (Tissue) }\end{array}$ & $\begin{array}{l}\text { Outcomes } \\
\text { (Process, e.g., Genes) }\end{array}$ & Publication \\
\hline $\begin{array}{l}\text { Control diet } \\
10 \% \text { sucrose } \\
10 \% \text { yeast } \\
5 \% \text { cornmea } \\
11 \% \text { agar } \\
3 \% \text { tegosept } \\
0.3 \% \text { propionic acid } \\
\text { HFD } \\
20 \%(w / v) \text { coconut oil }\end{array}$ & $\begin{array}{l}w^{1118} \\
\text { Females } \\
2-7 \mathrm{~d}\end{array}$ & $7 \mathrm{~d}$ & $\begin{array}{l}\text { Microarray } \\
\text { (heads) }\end{array}$ & $\begin{array}{l}\text { - Genes upregulated } \\
59 \text { genes } \\
\text { Odorant binding proteins } \\
\text { (Obp, OS9) } \\
\text { Odorant receptor coreceptor } \\
\text { (Orco) } \\
\text { Nucleotide metabolism (Gfat1, } \\
\text { Pepck2, (gogo, caps) } \\
\text { Stress responses (CG2837, } \\
\text { to, Cyp4e3) } \\
\text { Genes downregulated } \\
\text { 132 genes } \\
\text { DNA replicationMitosis } \\
\text { Cell cycle regulation } \\
\text { RNA binding } \\
\text { Neurogenesis } \\
\text { Oxidative stress (TotC, hsp26/27) } \\
\text { Neuronal level (CycA, brat, osk, } \\
\text { pum, orb, tun, RnrS, CG3699, } \\
\text { CG10962) } \\
\text { Neurophysiological } \\
\text { mechanisms (Fsn, lola, tyf) } \\
\text { Fat storage (dob) }\end{array}$ & Rivera et al. [157] \\
\hline
\end{tabular}


Table 4. Cont.

\begin{tabular}{|c|c|c|c|c|c|}
\hline Diet & Strain, Sex, Age & Time Period of Intervention & $\begin{array}{l}\text { Method } \\
\text { (Tissue) }\end{array}$ & $\begin{array}{l}\text { Outcomes } \\
\text { (Process, e.g., Genes) }\end{array}$ & Publication \\
\hline $\begin{array}{l}\text { Control diet } \\
\text { Food mix (New } \\
\text { Horizon Foods) } \\
\text { HFD } \\
20 \%(w / v) \text { coconut oil }\end{array}$ & $\begin{array}{l}w^{1118} \\
\text { Males, females } \\
3 \mathrm{~d}\end{array}$ & $7 \mathrm{~d}$ & $\begin{array}{l}\text { RNA sequencing (heads, } \\
\text { whole body) }\end{array}$ & $\begin{array}{l}\text { Results for males: } \\
\text { - Genes altered } \\
\text { Whole body: } 464 \text { genes } \\
\text { Head: } 143 \text { genes } \\
\text { Genes upregulated } \\
\text { Whole body: } \\
\text { Antioxidant capacity (Cyp4e3) } \\
\text { Hyperphagic behavior (to), } \\
\text { Lipid homeostasis (Cyp4e3, } \\
\text { CG5953) } \\
\text { Genes downregulated } \\
\text { Whole body: } \\
\text { Glycoside hydrolases } \\
\text { (LManIII-VI) } \\
\text { Heads: } \\
\text { Stress, immune response (TotA, } \\
\text { TotC, TotM) } \\
\text { Fat body: } \\
\text { Lipid homeostasis (Lsp2, Gnmt) } \\
\text { Results for females: } \\
\text { Genes altered } \\
\text { Whole body: } 330 \text { genes } \\
\text { Head: } 93 \text { genes } \\
\text { Genes upregulated } \\
\text { Whole body: } \\
\text { Antioxidant capacity (Cyp4e3) } \\
\text { Hyperphagic behavior (to), } \\
\text { Lipid homeostasis (Cyp4e3, } \\
\text { CG5953) } \\
\text { Head: } \\
\text { Lipid metabolism (Mtpa, yip2, } \\
\text { CG12262) } \\
\text { Genes downregulated } \\
\text { Fat body: } \\
\text { Lipid homeostasis (Lsp2, Gnmt) }\end{array}$ & Stobdan et al. [159] \\
\hline
\end{tabular}


Table 4. Cont.

\begin{tabular}{|c|c|c|c|c|c|}
\hline Diet & Strain, Sex, Age & Time Period of Intervention & $\begin{array}{l}\text { Method } \\
\text { (Tissue) }\end{array}$ & $\begin{array}{l}\text { Outcomes } \\
\text { (Process, e.g., Genes) }\end{array}$ & Publication \\
\hline $\begin{array}{l}\text { Control diet } \\
4 \% \text { sucrose } \\
2 \% \text { yeast } \\
4 \% \text { cornmea } \\
11 \% \text { agarose } \\
1 \% \\
\text { parahydroxybenzonate } \\
1 \% \text { propionic acid } \\
\text { HFD } \\
20 \%(w / v) \text { coconut oil }\end{array}$ & $\begin{array}{l}\text { Canton-S } \\
\text { Males, females } \\
5-10 \mathrm{~d}\end{array}$ & $7 \mathrm{~d}$ & $\begin{array}{l}\text { RNA sequencing (whole } \\
\text { body) }\end{array}$ & $\begin{array}{l}\text { - } \quad \text { Genes upregulated } \\
112 \text { genes } \\
\text { Long-chain fatty acid synthesis } \\
\text { (FASN2) } \\
\text { Genes downregulated } \\
91 \text { genes } \\
\text { Results for males: } \\
\text { - Genes upregulated } \\
\text { Molecular chaperone (CCT1) } \\
\text { Oxidative stress response } \\
\text { (Sod1, Sod2) } \\
\text { Genes downregulated } \\
\text { FA synthase (FASN1) } \\
\text { Unsaturated FA synthesis } \\
\text { (Desat1) } \\
\text { Drosophila insulin-like peptide } \\
\text { signaling pathway (tobi) } \\
\text { Immune response (Dro, DptA) } \\
\text { Results for females: } \\
\text { Genes upregulated } \\
\text { Unsaturated FA synthesis } \\
\text { (Desat1) } \\
\text { Glucose metabolism, } \\
\text { gluconeogenesis (sug) } \\
\text { Oxidative stress response } \\
\text { (Sod1, Sod2) } \\
\text { Genes downregulated } \\
\text { Drosophila insulin-like peptide } \\
\text { signaling pathway (tobi) } \\
\text { Glucose metabolism, } \\
\text { gluconeogenesis (Amy- } p, \\
\text { Amy-d) } \\
\text { Immune response (Dro, DptA) } \\
\text { Egg production (Vm26Aa, psd) }\end{array}$ & Azuma et al. [162] \\
\hline
\end{tabular}




\begin{tabular}{|c|c|}
\hline Upregulated transcription & Downregulated transcription \\
\hline $\begin{array}{l}\text { Metabolism \& energy provision } \\
\text { - Metabolic enzymes } \\
\text { - Gluconeogenesis } \\
\text { - Proteolysis } \\
\text { - Peptide processing } \\
\text { Infection \& stress response } \\
\text { - Heat shock proteins } \\
\text { - Cytochrome P450 } \\
\text { - Monooxygenase } \\
\text { - Chitin metabolic processing } \\
\text { Nuclear regulations } \\
\text { - Signal transduction } \\
\text { Sensory perception } \\
\text { - Odorant/pheromone proteins }\end{array}$ & $\begin{array}{l}\text { Metabolism \& energy provision } \\
\text { - Degradation of sugars \& waste } \\
\text { metabolites } \\
\text { - Nutrient storage } \\
\text { Development } \\
\text { - Neuromuscular signaling } \\
\text { - Neurogenesis } \\
\text { - Glycoside hydrolase (ळ) } \\
\text { Infection \& stress response } \\
\text { - Immunoglobulins } \\
\text { - Hemocyanin } \\
\text { Nuclear regulations } \\
\text { - RNA binding } \\
\text { - Mitosis } \\
\text { - DNA replication } \\
\text { - Cell cycle }\end{array}$ \\
\hline
\end{tabular}

Figure 13. Transcriptional changes in Drosophila melanogaster resulting from high-fat diet (HFD) feeding. An HFD induces upregulation (blue box) and downregulation (red box) of genes involved in metabolism and energy provision, infection and stress responses, nuclear regulation, sensory perception, and development (modified from [152,156,157,159,162]).

\subsection{Changes in Metabolome}

Only a few studies focusing on the effect of HSD or HFD intake in larvae or adult $D$. melanogaster on metabolomics were published in recent years.

Both the genotype (16\%) and the diet (9\%) considerably affect the metabolome of D. melanogaster, whereas the interaction of these two factors $(2 \%)$ apparently exerts minor effects on the variance of the fruit fly metabolome [163,164]. The presumed impacts of the ingestion of an HSD and HFD on D. melanogaster was investigated by metabolomics analyses, including information on the dietary intervention protocols and examples of upand downregulated metabolites. An overview is presented in Table 5.

\subsubsection{HSD Metabolome}

Colinet et al. (2013) performed a feeding study with wild-type D. melanogaster (a mixture of two wild populations collected in Brittany, France), which were consecutively administered diets containing sucrose, glucose, fructose and trehalose at concentrations between 0 and $1000 \mathrm{mM}$ from the larval to adult stages, and conducted metabolic fingerprinting by GC/MS 5 days post eclosion [130]. The sucrose- and fructose-containing diets modified, in particular, contain partially overlapping sets of metabolites of the carbohydrate metabolism. The elevated malate concentration, following the application of a sucrose- and fructose-containing diet, was suggested to be attributable for an altered lipid biosynthesis and a dose-dependently-increased fat accumulation. It was found that all sugar-supplemented diets were absorbed by the fruit flies and accumulated as fat. In addition, the intake of a glucose- and trehalose-enriched diet affected carbohydrate and amino acid metabolism. GABA concentrations dose-dependently decreased following the short-term ingestion of a glucose- or trehalose-supplemented diet. Low GABA concentrations affected the production and release of Dilps in insulin-producing cells [165]. Overall, a concentration-dependent increase in sorbitol level was detectable following ingestion of either diet, which resulted from activation of the polyol pathway due to the presence of 
high concentrations of fructose and glucose. Additionally, increased ROS production arose from modifications of the polyol pathway [130].

Simard et al. (2020) investigated the impact of the 15-day ingestion of an HSD containing $18 \%$ and $30 \%$ sucrose on the metabolite profiles of adult wild-type $w^{1118}$ flies and flies with mild mitochondrial pyruvate carrier (MPC) 1 deficiency using ${ }^{1} \mathrm{H}$ NMR spectroscopy [166]. The modification of a low number of metabolites, e.g., increases in fructose, glucose-6-phosphate and proline levels, as well as slight decreases in leucine, malate and citrate levels, was detected in $w^{1118}$ flies following the ingestion of the HSD. In contrast, flies with mild MPC1 deficiency exhibited an accumulation of numerous metabolites, such as sugars (e.g., glycogen, fructose, glucose), glycolysis intermediates (e.g., glucose-6-phosphate) and amino acids (e.g., glycine, proline, $\beta$-alanine). MPC is nested in the inner mitochondrial membrane and is essential for pyruvate transport into the mitochondria [167]. Sugar accumulation and the diminishment of pyruvate-stimulating respiration indicated metabolic inflexibility in MPC1-deficient flies, leading to a depletion of homeostasis [166].

Gillette et al. (2020) investigated the interaction of genes and diet in $w^{1118}$ and split ends (Spen)-depleted D. melanogaster larvae following treatment with an HSD (1 M corn syrup) via ultra-high-performance LC/MS. Spen, an extremely large, RNA-binding protein, is a key regulator of the energy balance in the fruit fly. Its depletion led to defects in energy metabolism (e.g., $\beta$-oxidation) and increases in TAG storage. In $w^{1118}$ flies, the total metabolic difference caused by the ingestion of an HSD amounted to a total of only $3.6 \%$ compared to the levels in controls. In general, the administration of an HSD led to a reduction in strain-dependent differences between Spen-depleted and $w^{1118}$ larvae. The similar metabolic profiles in both genotypes indicated that the HSD eradicated the genetic influence of Spen deficiency on metabolism [168].

Tuthill et al. (2020) analyzed the lipid profiles in the fat bodies, hemolymph and hearts of adult $w^{1118}$ D. melanogaster, following the ingestion of an HSD containing $1 \mathrm{M}$ sucrose, for three to five weeks by ultra-high performance liquid chromatography-MS/MS. The relative levels of diglycerides and TAG were increased in all analyzed tissues, with TAG being the most affected by the ingestion of an HSD. In addition, the lengths of substituent chains were modified following the intake of an HSD, resulting in fewer oddchain, esterified FAs in all examined tissues. The lower odd-chain FA content in HFD-fed fruit flies was discussed in the context of a concurrent change in the intestinal microbiome. Since odd-chain FA are generated by enteric bacteria, increased even:odd chain ratios may reflect a reduced lipogenesis by enteric bacteria. In the fat body, in general, the levels of 10 lipids increased, while those of 110 lipids decreased. The highest variability in lipid content was evident, with increases in saturated FA and monounsaturated FA substituents and a decrease in the relative proportion of double bonds. In the hemolymph, in total, the proportions of 33 lipids increased, while those of most of the lipids (131 lipids) decreased. The proportions of TAG and ether lipids changed to a smaller extent in the hemolymph than in the fat body. In addition, reductions in ceramides and sphingomyelin were evident in the hemolymph. Similarly, the alterations in cardiac tissues reflected the trends in the other tissues. In total, 44 lipids were altered in the cardiac tissue in response to the intake of an HSD [169].

Since the storage capacity of the fat body of $D$. melanogaster is limited, a surplus of FA/lipids elicits lipotoxicity in other tissues [170]. 
Table 5. Overview of dietary intervention studies regarding changes in metabolome following high-sugar diet (HSD) or high-fat diet (HFD) feeding in Drosophila melanogaster. The studies are ordered by release date.

\begin{tabular}{|c|c|c|c|c|c|}
\hline Diet & Strain, Sex, Age & Time Period of Intervention & Method & Outcomes & Publication \\
\hline HSD & & & & & \\
\hline $\begin{array}{l}\text { Control diet } \\
10,400 \mathrm{mM} \text { sucrose, } \\
\text { fructose, glucose or } \\
\text { trehalose } \\
8 \% \text { yeast } \\
1.5 \% \text { agar }\end{array}$ & $\begin{array}{l}\text { Mix of two wild } \\
\text { populations } \\
\text { Females } \\
5 \mathrm{~d}\end{array}$ & Egg to $5 \mathrm{~d}$ adult females & $\begin{array}{l}\text { Gas chromatography/mass } \\
\text { spectrometry }\end{array}$ & $\begin{array}{l}\text { Results for sucrose: } \\
\text { - Metabolite level } \\
\text { increased } \\
\text { Sucrose } \\
\text { Fructose } \\
\text { Sorbitol } \\
\text { Malate }\end{array}$ & Colinet et al. [130] \\
\hline $\begin{array}{l}\text { HSD } \\
1000 \mathrm{mM} \text { sucrose, fructose, } \\
\text { glucose or trehalose }\end{array}$ & & & & $\begin{array}{l}\text { Results for fructose: } \\
\text { - } \quad \text { Metabolite level } \\
\text { increased } \\
\text { Fructose } \\
\text { Trehalose } \\
\text { Malate } \\
\text { Results for glucose: } \\
\text { - Metabolite level } \\
\text { increased } \\
\text { Whole-body metabolite } \\
\text { concentrations } \\
\text { Sorbitol } \\
\text { Inositol } \\
\text { Amino acids (e.g., valine, leucine, } \\
\text { isoleucine) } \\
\text { Metabolite level } \\
\text { decreased } \\
\text { Maltose } \\
\text { Gamma-amino-butyric acid } \\
\text { (GABA) }\end{array}$ & \\
\hline
\end{tabular}


Table 5. Cont.

\begin{tabular}{|c|c|c|c|c|c|}
\hline Diet & Strain, Sex, Age & Time Period of Intervention & Method & Outcomes & Publication \\
\hline & & & & $\begin{array}{l}\text { Results for trehalose: } \\
\text { - Metabolite level } \\
\text { increased } \\
\text { Sorbitol } \\
\text { Trehalose } \\
\text { Amino acids } \\
\text { (e.g., valine, leucine, isoleucine) } \\
\text { Metabolite level } \\
\text { decreased } \\
\text { Galactose } \\
\text { GABA }\end{array}$ & \\
\hline Control diet & $w^{1118}$, split ends & First to late third instar larvae & Liquid & Results for $w^{1118}$ & Gillette et al. [168] \\
\hline $1 \%$ corn syrup & (Spen)-depleted & & chromatography/mass & Metabolic change: & \\
\hline $4 \%$ yeast & D. melanogaster & & spectrometry & $4 \%$ of the analyzed metabolites & \\
\hline $4 \%$ malt extract & & & & were changed & \\
\hline $7 \%$ cornmeal & Males, females & & & & \\
\hline $1 \%$ soy flour & & & & Results for Spen-depleted flies: & \\
\hline $0.4 \%$ propionic acid & & & & $7 \%$ of the analyzed metabolites & \\
\hline $0.8 \%$ tegosept & $22-24 \mathrm{~h}$ & & & were changed & \\
\hline $\begin{array}{l}\text { HSD } \\
18 \% \text { corn syrup }\end{array}$ & & & & $\begin{array}{l}\text { Metabolite level } \\
\text { decreased } \\
\text { L-carnitine }\end{array}$ & \\
\hline
\end{tabular}


Table 5. Cont.

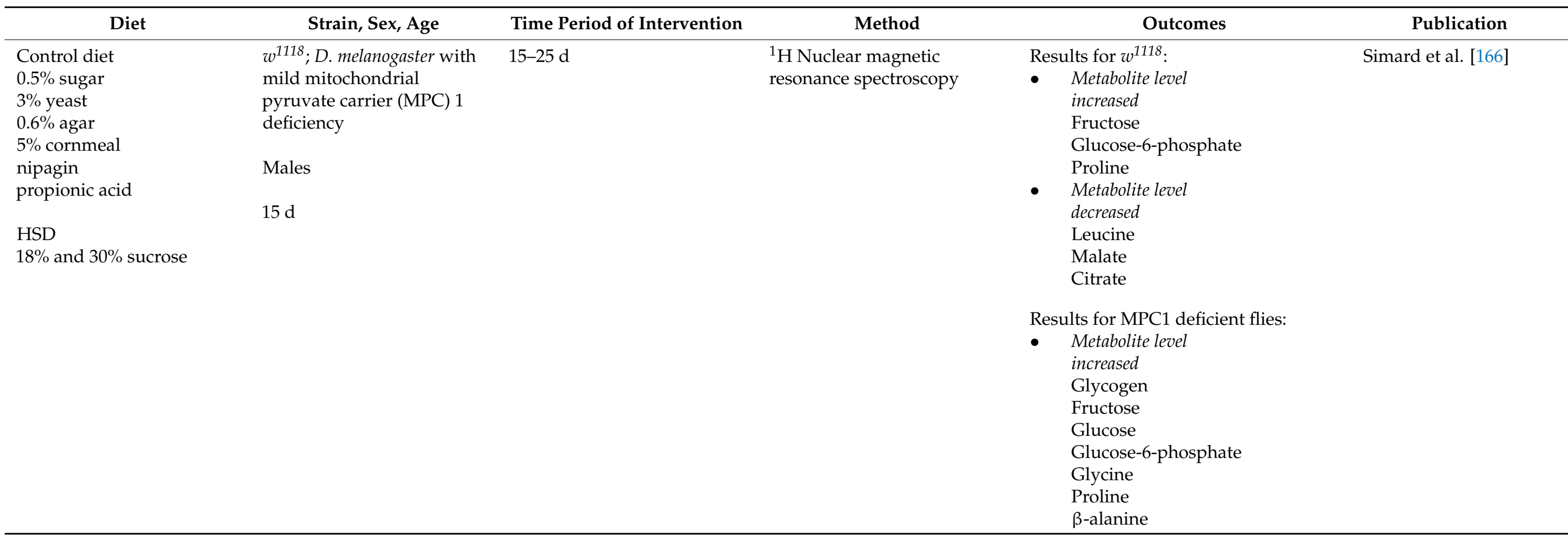


Table 5. Cont.

\begin{tabular}{|c|c|c|c|c|c|}
\hline Diet & Strain, Sex, Age & Time Period of Intervention & Method & Outcomes & Publication \\
\hline $\begin{array}{l}\text { Control diet } \\
5 \% \text { sugar } \\
10 \% \text { yeast } \\
2 \% \text { peptone } \\
1 \% \text { agar } \\
\text { HSD } \\
34 \% \text { sucrose }\end{array}$ & $\begin{array}{l}w^{1118} \\
\text { Males, females } \\
1 \mathrm{~d}\end{array}$ & $3-5$ weeks & $\begin{array}{l}\text { Ultra-high performance } \\
\text { liquid chromatography/mass } \\
\text { spectrometry/mass } \\
\text { spectrometry }\end{array}$ & $\begin{array}{l}\text { Results for fat body: } \\
\text { - } \quad \text { Metabolite level increased } \\
\text { (10 lipids) } \\
\text { Diglycerides (DAG) } \\
\text { Triacylglycerides (TAG) } \\
\text { Saturated fatty acid, } \\
\text { monounsaturated fatty acid (FA) } \\
\text { substituents (e.g., lauric acid, } \\
\text { myristic acid, palmitic acid) } \\
\text { Relative proportion of double } \\
\text { bonds } \\
\text { Even:odd chain ratios } \\
\text { Plasmenyl TAGs (DAGEs) } \\
\text { Other plasmenyl, } \\
\text { plasmanyl species } \\
\text { Palmitate } \\
\text { Metabolite level } \\
\text { decreased } \\
\text { 110 lipids } \\
\text { Odd-chain esterified FAs } \\
\text { Results for hemolymph: } \\
\text { Metabolite level } \\
\text { increased } \\
\text { (33 lipids) } \\
\text { DAG } \\
\text { TAG } \\
\text { Glycerolipids } \\
\text { Even:odd chain ratios } \\
\text { Saturated FA substituents } \\
\text { DAGEs } \\
\text { Lyso-phosphatidylcholines }\end{array}$ & Tuthill et al. [169] \\
\hline
\end{tabular}


Table 5. Cont.

Diet Strain, Sex, Age

Time Period of Intervention

Method

Outcomes

Publication

- Metabolite level

decreased

(131 lipids)

Odd-chain esterified FAs

Phospholipid

Unsaturated substituents

Ceramides

Sphingomyelin

Other plasmenyl

plasmanyl species

Results for heart:

Altered: 44 lipids

- Metabolite level

increased

DAG

TAG

Chained TAGs

Chained DAGEs

Glycerolipids

Palmitate

- Metabolite level

decreased

Odd-chain esterified FAs

Odd-chain substituents

PUFA substituents

Other plasmenyl,

plasmanyl species 
Table 5. Cont.

\begin{tabular}{|c|c|c|c|c|c|}
\hline Diet & Strain, Sex, Age & Time Period of Intervention & Method & Outcomes & Publication \\
\hline $\begin{array}{l}\text { Control diet } \\
\text { yeast } \\
\text { corn starch } \\
\text { molasses } \\
\text { HFD } \\
20 \%(w / v) \text { coconut oil }\end{array}$ & $\begin{array}{l}w^{1118} \\
\text { Females } \\
3-5 \mathrm{~d}\end{array}$ & $7 \mathrm{~d}$ & $\begin{array}{l}\text { Gas chromatography/mass } \\
\text { spectrometry }\end{array}$ & $\begin{array}{l}\text { Metabolite level } \\
\text { increased } \\
\text { Total fatty acids } \\
\text { (e.g., palmitate, oleate, stearate) } \\
\text { Lactate } \\
\text { Pyruvate } \\
\text { Urea } \\
\text { Uric acid } \\
\text { Acetyl-coenzyme A } \\
\text { (acetyl-CoA) } \\
\text { Metabolite level } \\
\text { decreased } \\
\text { Fumarate } \\
\text { Malate } \alpha \text {-ketoglutarate } \\
\text { Numerous amino } \\
\text { acids }\end{array}$ & Heinrichsen et al. [156] \\
\hline $\begin{array}{l}\text { Control diet } \\
\text { Cornmeal-molasses food } \\
\text { HFD } \\
3 \%(w / v) \text { coconut oil }\end{array}$ & $\begin{array}{l}16 \text { different cultured } \\
\text { genotypes } \\
\text { Larvae } \\
\text { First instar }\end{array}$ & First to late third instar larvae & $\begin{array}{l}\text { Liquid } \\
\text { chromatography/mass } \\
\text { spectrometry } \\
\text { Gas chromatography/mass } \\
\text { spectrometry }\end{array}$ & $\begin{array}{l}\text { Metabolite level } \\
\text { increased } \\
\text { Medium-chain FAs } \\
\text { (caproate, caprylate, caprate, } \\
\text { laurate) } \\
\text { Dicarboxylic FAs } \\
\text { (decanedioate, dodecanedioate) } \\
\text { Monohydroxy FAs } \\
\text { (3-hydroxysebacate, } \\
\text { 3-hydroxydecanoate) } \\
\text { Glucose-6-phosphate } \\
\text { Fructose-6-phosphate Citrate } \\
\text { Dipeptides }\end{array}$ & Oza et al. [171] \\
\hline
\end{tabular}


Table 5. Cont.

\begin{tabular}{|c|c|c|c|c|c|}
\hline Diet & Strain, Sex, Age & Time Period of Intervention & Method & Outcomes & Publication \\
\hline $\begin{array}{l}\text { Control diet } \\
1 \% \text { light corn syrup } \\
4 \% \text { yeast } \\
4 \% \text { light malt extract } \\
7 \% \text { yellow cornmeal } \\
1 \% \text { soy flour } \\
0.5 \% \text { agar } \\
0.4 \% \text { propionic acid } \\
0.8 \% \text { tegosept } \\
\text { HFD } \\
15 \%(w / v) \text { coconut oil }\end{array}$ & $\begin{array}{l}w^{1118} \text {; split ends } \\
\text { (Spen)-depleted } D . \\
\text { melanogaster } \\
\text { Males, females } \\
\text { Larvae } \\
22-24 \mathrm{~h}\end{array}$ & First to late third instar larvae & $\begin{array}{l}\text { Liquid } \\
\text { chromatography/mass } \\
\text { spectrometry }\end{array}$ & $\begin{array}{l}\text { Results for } w^{1118} \\
\text { - } \quad \text { Metabolic change: } \\
11 \% \text { of the analyzed metabolites } \\
\text { were changed } \\
\text { Results for Spen-depleted flies: } \\
\text { - Metabolic change: } \\
24 \% \text { of the analyzed metabolites } \\
\text { were changed } \\
\text { Metabolite level } \\
\text { decreased } \\
\text { Acetylcholine } \\
\text { L-carnitine }\end{array}$ & Gillette et al. [168] \\
\hline $\begin{array}{l}\text { Control diet } \\
0.5 \% \text { agar } \\
0.6 \% \text { sugar } \\
3 \% \text { yeast } \\
5 \% \text { cornmeal } \\
\text { nipagin } \\
\text { propionic acid } \\
\text { HFD } \\
20 \%(w / v) \text { coconut oil }\end{array}$ & $\begin{array}{l}w^{1118} \\
\text { Males } \\
10 \mathrm{~d}\end{array}$ & $10 \mathrm{~d}$ & $\begin{array}{l}\text { Nuclear magnetic resonance } \\
\text { spectroscopy }\end{array}$ & $\begin{array}{l}\text { Metabolite level } \\
\text { increased } \\
25 \text { metabolites } \\
8 \text { amino acids (serine, } \\
\text { valine, tyrosine) } \\
\text { Glycogen } \\
\text { Glucose } \\
\text { Glucose-6-phosphate } \\
\text { Pyruvate } \\
\text { Citrate } \\
\text { Fumarate } \\
\text { Succinate } \\
\text { Malate } \\
\text { Cytosolic nicotinamide-adenine } \\
\text { dinucleotide } \\
\text { Acetyl-CoA }\end{array}$ & Cormier et al. [172] \\
\hline
\end{tabular}


It was also shown, in the previously mentioned study of Tuthill et al. (2020), that a long-term (3- to 5-week) intake of an HSD led to an overabundance of lipids in the fat body and heart and increased levels of palmitate in lipids, di-saturated glycerolipids and DAGEs, which were linked to lipotoxic effects. Additionally, an increase in the even:odd chain ratio following the ingestion of an HSD was evident in all tissues studied, which was again related to the alteration of the gut microbiota [169]. A summary of the outlined results following a HSD is depicted in Figure 14.

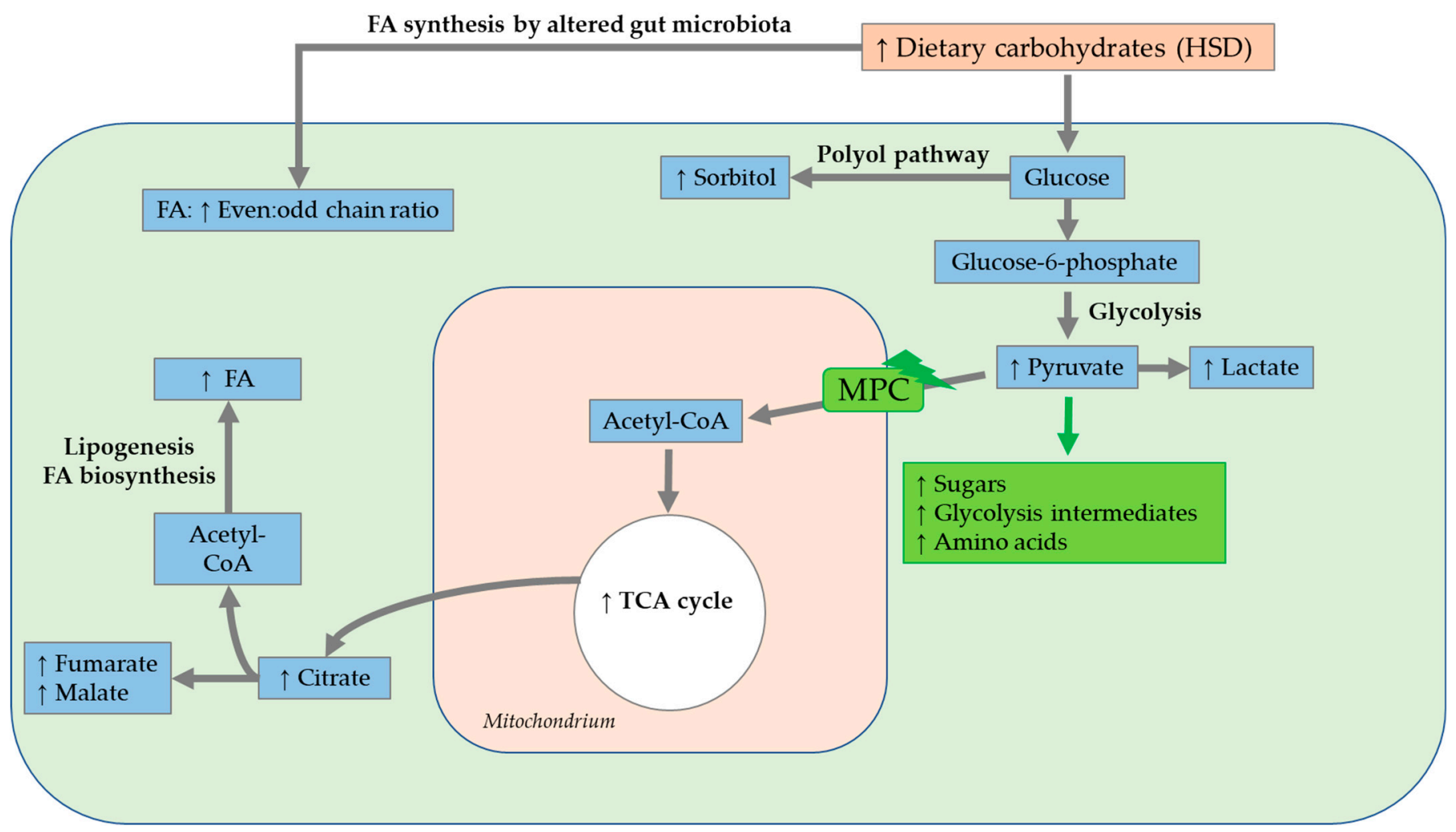

Figure 14. Metabolomic changes in Drosophila melanogaster in response to high-sugar diet (HSD) feeding. Flies fed an HSD supplemented with sucrose show elevated levels of pyruvate and lactate, which can be explained by stimulation of the glycolysis pathway. Moreover, the levels of fatty acids (FA) and stored lipids are increased, indicative of enhanced lipogenesis and FA biosynthesis $[130,169]$. Additionally, flies fed an HSD exhibit fewer odd-chain esterified FAs in all examined tissues, which is most likely attributable to an altered composition of the gut microbiota [169]. Ingestion of an HSD leads to increased levels of sorbitol produced via glucose utilization in the polyol pathway [130]. Effects elicited by long-term feeding on an HSD are depicted with green symbols and boxes. Long-term HSD exposure negatively affects the mitochondrial pyruvate carrier (MPC) in the inner mitochondrial membrane (lightning bolt). Mild MPC deficiency is associated with increased accumulation of sugars, glycolysis intermediates and amino acids (green box), and a reduction in pyruvate-stimulating respiration suggests metabolic inflexibility and the disruption of homeostasis [166] (modified from [130,166,169]). Acetyl-CoA, acetyl-coenzyme A; TCA, tricarboxylic acid; $\uparrow$ increase; $\downarrow$ decrease; thickness of arrows indicates flux rate.

\subsubsection{HFD Metabolome}

Oza et al. (2019) conducted untargeted metabolomics analyses (GC/MS and LC/MS) of the larval $D$. melanogaster of 16 different cultured genotypes. Larval feeding of an HFD containing $3 \%(w / v)$ coconut oil caused increased medium-chain FA and dicarboxylic FA levels in larvae, which presumably originated directly from coconut oil in the HFD. Ingestion of the HFD led to increased monohydroxy FA levels as end products of the omega oxidation pathway. Medium-chain FAs of the HFD were incorporated directly into the 
larval omega FA oxidation pathway as an alternative to $\beta$-FA oxidation. Additionally, larval intake of the HFD increased glucose-6-phosphate, fructose-6-phosphate and citrate levels, suggesting the involvement of glycolysis and the downstream TCA cycle. Larvae of the different cultured genotypes represented different TAG storage phenotypes with differing dipeptides, lysolipids and components of amino acid metabolism. The intake of the HFD led to increased protein catabolism or enzymatic dipeptide synthesis with increased dipeptide levels. In total, a higher network density was evident after larval ingestion of the HFD than after the intake of a control diet [171].

Heinrichsen et al. (2014) performed metabolomics analyses (GC/MS) on female $w^{1118}$ D. melanogaster following the ingestion of an HFD supplemented with $20 \%(w / v)$ coconut oil for 7 days. The intake of the HFD led to increases in the total metabolic rate and oxygen consumption and decreases in fumarate, malate and $\alpha$-ketoglutarate related to the TCA cycle. The proportions of total FAs, lactate, pyruvate, urea and uric acid were increased, while those of numerous amino acids were decreased, which indicated alterations in carbohydrate and amino acid metabolism. Additionally, the ingestion of the HFD increased FA oxidation in mitochondria, leading to increased levels of acetyl-coenzyme A (acetylCoA). As pyruvate is not primarily used for acetyl-CoA synthesis, pyruvate accumulation and conversion to lactate occurred. In parallel, amino acid oxidation was increased to provide anaplerotic substrates for the TCA cycle. This alteration in nitrogen metabolism was also evident at the transcriptional level with regard to the expression of CG9510 (see section Transcriptomics: Application to D. melanogaster) [156].

Cormier et al. (2021) validated the abovementioned findings of Heinrichsen et al. (2014), since a high-substrate supply of FAs, provided by the intake of an HFD containing $20 \%(w / v)$ coconut oil for 10 days, caused an overload of the TCA cycle with increased levels of cytosolic nicotinamide-adenine dinucleotide (NADH) and acetyl-CoA in male $w^{1118}$ flies, leading to an inhibition of pyruvate usage and increased pyruvate accumulation. TCA cycle intermediates and amino acids replenished the TCA cycle. In total, metabolomics analyses (NMR spectroscopy) indicate an increase in TCA cycle-related metabolites, as well as an accumulation of seven metabolites associated with carbohydrate metabolism and eight amino acids following the 10-day ingestion of an HFD. The accumulation of 25 metabolites totally combined with mitochondrial dysfunctions suggests a metabolic of an inflexibility following the intake of a HFD [172].

Apart from testing an HSD (mentioned above), Gillette et al. (2020) fed $w^{1118}$ and split ends (Spen)-depleted D. melanogaster larvae with an HFD (15\% ( $w / v)$ coconut oil), before conducting metabolome analyses via ultra-high-performance LC/MS. In $w^{1118}$ flies, the total metabolic difference caused by ingestion of the HSD amounted to a total of only $3.6 \%$ compared to the level in controls, while the difference following the intake of an HFD was comparatively high at $11.1 \%$. The $w^{1118}$ flies demonstrated a higher metabolic flexibility and substantial resistance to dietary extremes such as high-fat intake, compared to the Spen-depleted flies. In general, the administration of an HSD and HFD led to a reduction in strain-dependent differences between Spen-depleted and $w^{1118}$ larvae. Thus, only two metabolites, acetylcholine and L-carnitine, among 140 detected metabolites differed in HFDversus control diet-fed flies. The similar metabolic profiles in both genotypes indicated that the HSD and HFD eradicated the genetic influence of Spen deficiency on metabolism [168]. A summary of the outlined results following a HFD is shown in Figure 15. 


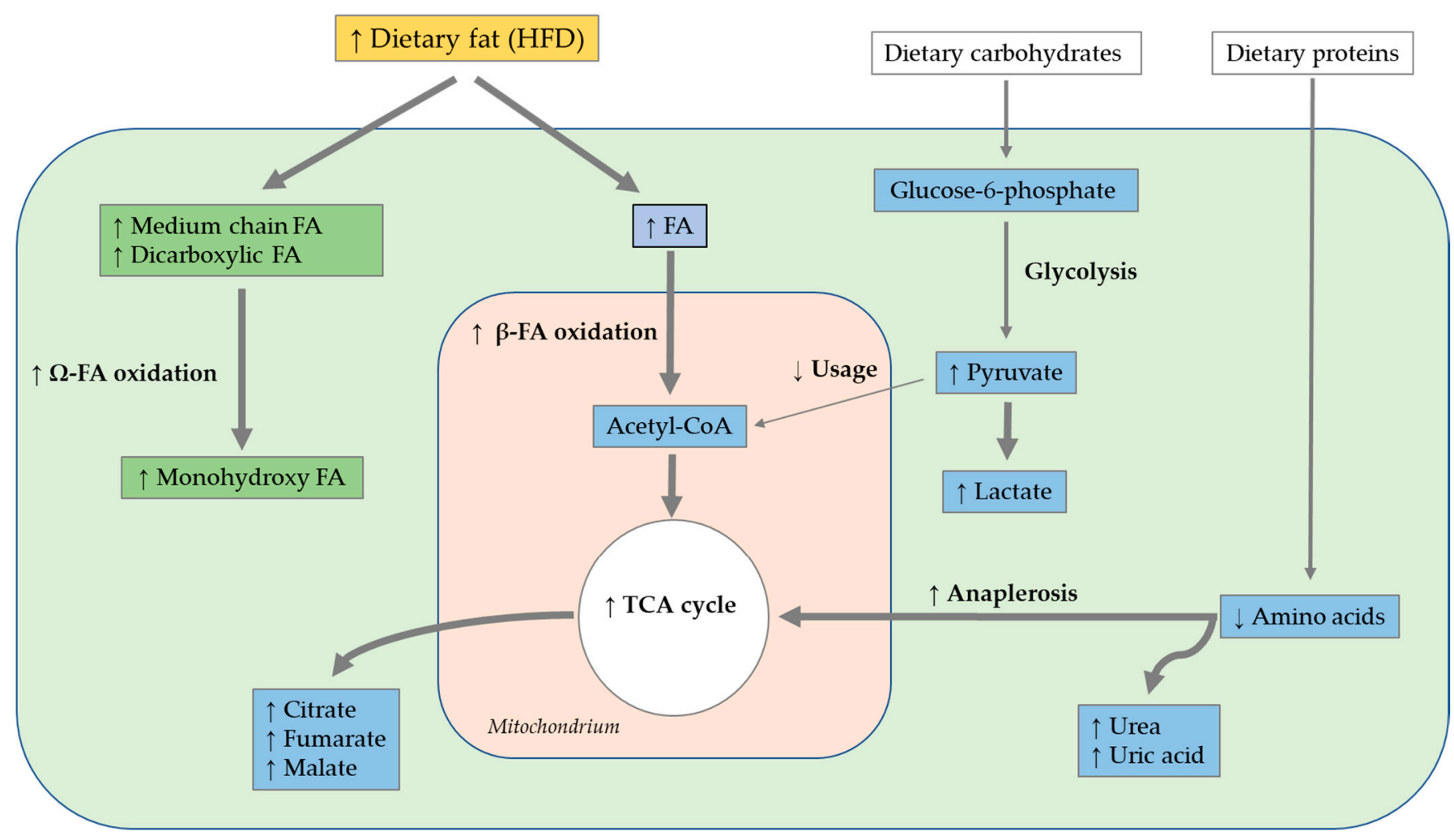

Figure 15. Metabolomic changes in Drosophila melanogaster fed a high-fat diet (HFD). Metabolites that are affected in adult flies are in blue boxes, and those that are specifically altered in larvae are in green boxes. In adult flies, an HFD led to enhanced levels of acetyl-coenzyme A (acetyl-CoA), indicating an increased $\beta$ - oxidation of fatty acids (FAs). In line with this, citrate, malate and fumarate concentrations are increased, which points to the stimulation of the tricarboxylic acid (TCA) cycle, fueled by the acetyl-CoA surplus. An overload of the TCA cycle by $\beta$-oxidation-derived acetyl-CoA is further supported by elevated levels of pyruvate and lactate, indicating a decreased utilization of glycolysis products by the TCA cycle $[156,172]$. In addition, amino acid levels declined, and products of amino acid catabolism, such as urea and uric acid, were elevated. This points to an increased demand for amino acids to provide anaplerotic metabolites for the TCA cycle [172]. In larval D. melanogaster (green boxes), an increase in $\Omega$ - FA oxidation is also evident [171] (modified from $[156,171,172]) . \uparrow$ increase; $\downarrow$ decrease; arrow thickness indicates flux rate.

\section{Relevance of Findings in D. melanogaster for Other Species}

Comprehensive phenotyping of the model organism $D$. melanogaster is feasible in the context of chronic metabolic diseases such as obesity and diabetes. To what extent findings in D. melanogaster may be translated to other mammalian species, such as laboratory rodents and humans, will be discussed in the section below.

\subsection{Lipid Metabolism}

The evolutionary and/or functional conservation of multiple tissue and organ structures, such as the central nervous system and the digestive tract, reveals similarities in signaling pathways and regulation of lipid metabolism between $D$. melanogaster and mammalian species.

In D. melanogaster, the digestion and absorption of lipids predominantly proceed in the midgut, which can be broadly subdivided into the anterior, middle and posterior midgut and by morphology into 10-14 subregions [173]. In metazoan animals, ingested macronutrients, including lipids and phospholipids, must be enzymatically degraded within the alimentary tract before they can be taken up by enterocytes. Although the intestinal digestion of dietary lipids by the fruit fly is still poorly understood, the current data 
indicate that crucial aspects are conserved with respect to the situation in mammals. The Drosophila genome encodes several putative lipases exhibiting midgut expression [174]; currently, only a single intestinal TAG lipase, Magro, has been functionally described [175,176]. Nevertheless, it is highly likely that, similar to gastric, lingual and pancreatic lipases in mammals, ingested lipids (TAGs, phospholipids) are initially hydrolyzed by different lipases, namely neutral and acidic lipases in the fruit fly. Based on studies with other insects, it can be also assumed that lipid digestion and absorption in Drosophila is promoted by emulsification through the generation of FA-amino acid complexes, glycolipid complexes and FA-lysophospholipid micelles [177]. However, bile salts, which represent the key emulsifiers in mammalian dietary lipid digestion, are lacking in the fruit fly. In mammals, the lipase products monoacylglycerol (MAG), DAG and free FAs form mixed micelles with lysophosphatidic acid, cholesterol, bile salts and fat-soluble vitamins capable of diffusing into enterocytes. In addition, MAG and free FAs pass the enterocyte membrane presumably by passive diffusion and active transport and are re-esterified back into TAGs. Similarly, free FAs, MAG, DAG and sterols are absorbed through the Drosophila midgut epithelium most likely facilitated by the emulsification and/or driven by diffusion via a concentration gradient caused by lipid re-synthesis in enterocytes. Whether transporter proteins are involved in this process has not yet been shown. A surplus of dietary lipids can result in TAG accumulation and transient storage within cytosolic lipid droplets of the enterocytes [178,179]. Additionally, FAs are produced in enterocytes via tissue-autonomous de novo lipogenesis, primarily by the enzymes acetyl-CoA-carboxylase and FA synthase, from acetyl-CoA (e.g., excess dietary carbohydrates). However, unlike in mammals, the de novo biosynthesis of cholesterol was not observed in D. melanogaster. Hence, the fruit fly is a cholesterol auxotroph and depends on the dietary intake of cholesterol mediated by Niemann-Pick-type C-1b [180,181]. FAs from the diet and de novo lipogenesis are converted via the glycerol-3-phosphate pathway or, alternatively, the MAG pathway to DAG.

For the interorgan transport of lipid molecules, such as FAs, DAG, TAG and sterols, from enterocytes to other tissues via the blood/lymph or hemolymph system, the presence of specific lipid-binding proteins is required. In D. melanogaster, DAG, cholesterol, cholesteryl ester and phospholipids are loaded onto the apoB-family lipoprotein, lipophorin, mediated by another apoB-family lipoprotein, a lipid transfer particle, before the resulting lipoprotein complex is secreted into hemolymph [181,182]. In mammals, in intracellular metabolism, TAGs combine with cholesterol and apolipoprotein to form chylomicrons, which are then released via the lymphatic system into the blood circulation. In contrast, short- and middle-chain FAs are directly bound to albumin and are transported in the blood circulation without re-esterification. The lipophilic substances in the lymphatic system and blood circulation proceed to be transported as lipoproteins, which are distinguished by different apoproteins, each intended for specific transport particles, enzymes and receptor binding in tissues. At endothelial cell membranes, TAGs in chylomicrons undergo cleavage catalyzed by lipoprotein lipase into glycerol and FAs. The FAs can be directly transferred to muscle for energy synthesis or to adipose tissue for storage. Fat storage occurs primarily by re-esterification into TAGs in white adipose tissue located both intra-abdominally and subcutaneously [183]. The chylomicron remnants that remain after hydrolysis are metabolized in the liver. In addition, under conditions of carbohydrate excess, de novo lipogenesis mainly occurs via the acetyl-CoA-carboxylase and FA synthase enzymes from acetyl-CoA in adipose tissue and liver tissue [184]. The lipophilic products from endo- and exogenous supplies are transported as very-low-density lipoproteins (VLDL) in the bloodstream and undergo cleavage catalyzed by lipoprotein lipase. After the hydrolysis of VLDL by lipoprotein lipase, intermediate-density lipoproteins remain, which are further converted into low-density lipoproteins. These mainly transport cholesterol to the cells. Excess cholesterol and phospholipids are shuttled back to the liver by high-density lipoprotein $[185,186]$.

The accumulation of energy reserves stored as fat enables energy to be available on demand in periods of nutritional deficiency. The fat mobilization process is highly conserved among fruit flies and mammals. In mammals, lipolysis involves all tissues (e.g., 
brown and white adipose tissue, muscle tissue and heart tissue), with most TAG storage in white adipose tissue. In energy-deficit conditions, cyclic adenosine monophosphate binds to protein kinase A (PKA), which catalyzes the phosphorylation of hormone-sensitive lipase (HSL) and perilipin 1. The phosphorylation of perilipin 1 leads to increased recruitment of HSL at the lipid monolayer of the lipid droplets, where lipolysis is stimulated. Adipose TAG lipase (ATGL) hydrolyzes TAGs into DAG, and some FAs play an important role in $\beta$ oxidation $[187,188]$. DAG is then hydrolyzed by Hsl into MAG, which in turn is hydrolyzed by monoacylglycerol lipase into glycerol and FAs. The FAs can be utilized for energy synthesis, re-esterification into TAGs and the synthesis of, for example, phospholipids and cholesteryl esters [186].

In the fruit fly, energy requirements can be met by the lipolysis of stored TAGs primarily in the fat body by the lipase BRUMMER, a homolog of human ATGL. Alternatively, other TAG lipases, which are dependent on adipokinetic hormone signals, can be involved in lipolysis. Similar to mammals, lipolysis is regulated by the PAT (perilipin, ADRP, TIP47) domain proteins, PLIN1 and PLIN2. In energy-deficit conditions, PLIN1 is phosphorylated by PKA, leading to the recruitment of Hsl at the lipid monolayer of lipid droplets and the stimulation of lipolysis [187]. The resultant DAG can then be hydrolyzed into FAs, possibly by Drosophila Hsl, to become available for $\beta$-oxidation and synthesis [179].

\subsection{Obesity and Related Comorbidities}

Obese Drosophila phenotypes can be generated by either genetic or dietary means. The genetic factors influencing obesity include genetic variations such as the alteration of a specific gene (monogenic factors), multiple genes (polygenic factors) or a combination of obesity with other organic or systemic dysfunctions (syndromic factors) [189]. Based on the evolutionary conservation of genes, findings from genetic studies on D. melanogaster may be at least partly transferred to other species such as mammals, especially in the context of disease association. For example, the downregulation of the genes $\mathrm{bmm}$ (a homolog of $A T G L)$ and $A K H R$ in D. melanogaster was shown to disturb fat homeostasis, resulting in the inhibition of the release of stored fat, causing obesity under unchanged dietary conditions [176,190]. Similarly, investigations with laboratory rodents and human studies indicated a connection between the downregulation of the ATGL gene, for example, by a pharmacological ATGL inhibitor and the accumulation of TAGs in various tissues [191].

Following industrialization and modernization, a "Westernization" of lifestyle and diet has occurred in humans, characterized by a decrease in daily physical activity and intake of an energy-dense Western diet rich in processed foods, meat, dairy products and sweets [192,193]. In this regard, the daily energy intake per person among human populations worldwide has increased over the last 30-50 years [194]. A permanent excess of energy intake due to consumption of energy-dense foods, accompanied by poor physical activity, causes overweight, obesity and comorbidities in humans [195-198] and animals [199-201]. Similarly, the obesity phenotypes in D. melanogaster induced by HSD and HFD feeding are accompanied by increased fat storage [22,75,128], alterations in carbohydrate-insulin homeostasis $[124,129,141]$, and reductions in fitness $[8,22,134]$ and reproductive capacity $[75,137,142]$.

The Western diet, which has a combination of high levels of fat, sugar and salt, decreases lifespan, reproduction, mitochondrial function and activity; increases TAG storage; and has stronger detrimental effects than an HSD or HFD alone. There is also evidence that the exposure of $D$. melanogaster to a Western diet causes alterations in food-seeking behavior and choice, increasing the preference of flies for a diet rich in sugar, fat and salt over the standard diet [202].

A preference for fat and sugar [203,204] and reduced taste perception [205,206] (reversible by weight loss [207]) are also found in overweight humans. However, the causeeffect relationship between these associations is mostly unclear [208].

The impact of fat quantity in the diet on the development of obesity and related diseases is widely discussed. In guidelines and recommendations in many countries, the 
total fat intake is considered, and reference levels of 30 percent of energy per day are set as far as human nutrition is concerned [209,210]. Overall, with probable evidence, no association between total fat intake and obesity is observed when energy adjustment is applied [211].

A study on rats and D. melanogaster found that a moderate HFD with 35 energy percent, compared with an isocaloric control diet without added fat, was unlikely to increase body weight and TAG storage and had a positive effect on lifespan. The isocaloric moderate HFD caused a downregulation of anabolism and upregulation of catabolism of free FAs, resulting in a downregulation of free FAs in serum and tissues. The downregulation of palmitic acid in turn upregulated the peroxisome, proliferator-activated receptor gamma (PPARG)/PPARG-related coactivator 1 (PPRC1) expression, which resulted in decreases in oxidative stress and inflammation, improving the survivorship of rats and fruit flies [212].

Life circumstances such as limited sleep, alterations in circadian rhythm (e.g., shift work), stress and intense emotions also influence eating behavior and metabolism and promote obesity.

In $D$. melanogaster, it was shown that stress induced by prolonged social isolation causes an increase in food intake and a reduction in sleep. Social isolation results in an increase in limostatin and a reduction in drosulfakinin at the transcriptional level in the head, suggesting that social isolation mimics a starvation state without a limitation of food supply [213]. Similar observations of changes in eating behavior caused by social isolation and emotional stress toward uncontrolled food intake, increased food cravings, emotional eating, binge-like eating, and, to some extent, increased restrictive eating, were obtained in laboratory rodents $[214,215]$ and humans [216,217], predominantly in females.

Moreover, a circadian rhythm of food intake can be identified in D. melanogaster. Regulated by clock genes, food intake is concurrent with locomotion rhythms occurring around sunrise and sunset. Mutations in clock genes induce a shift in meal timing without affecting the overall quantity of food eaten [218]. Additionally, a shift in circadian rhythm in fruit flies causes sex- and line-dependent changes in glycogen and TAG content, with Oregon females demonstrating increased TAG levels [219].

Circadian rhythms of gene expression, enzyme and hormone release, transport systems, and consequently, metabolic regulation can similarly be seen in mammals. Mutations in, e.g., clock genes, also cause reductions in diurnal feeding rhythms, which can promote obesity and metabolic diseases [220,221]. For example, disturbed rhythmic insulin secretion from the pancreas predisposes mammals to diabetes [222].

In addition, it was demonstrated in laboratory rodents that an HFD provided ad libitum impaired the circadian expression of, for example, the genes, clock and period circadian regulator (per1), and promoted obesity. Feeding a temporary HFD in a fixed time frame (e.g., zeitgeber phase $4-8$ as CT0 lights on) recovers the gene expression of clock genes and can be used for obesity prevention [221].

Diabetes mellitus can be modeled in D. melanogaster, with type 2 diabetes concomitant with obesity. The insulin signaling pathway in the fruit fly is evolutionarily conserved. D. melanogaster produces eight different Dilps, of which Dilp1, 2, 3 and 5 [223] were demonstrated to have a function homologous to that of vertebrate insulin and act in various target tissues. Dilp6 is most similar to mammalian insulin-like growth factors. In the adult fly, Dilps are produced by insulin-producing cells of the central nervous system, midgut, renal tubules, adipose tissue, ovaries and abdominal ganglion [224].

Circulating Dilps in the hemolymph bind to the Drosophila insulin receptor, causing the activation of chico, a homolog to adaptor protein insulin receptor substrate 1-4 in mammals. Activated chico interacts with two distinct Src homology 2 domain proteins, leading (homologous to the process in mammals) to the activation of two different cascades of the insulin signaling pathway (via extracellular-signal-regulated kinase-A and DAKTdS6 kinase) [225-228]. 
The insulin signaling pathway interacts with a transcriptional feedback control system of $d T O R$ and $d F O X O$ with a systemic and tissue-autonomous impact on metabolic adaptation, for example, in response to varying food supply [229].

After exposure to an HSD, D. melanogaster demonstrates hyperglycemia, resulting in increased Dilp2, 3 and 5 expression [129]. The prolonged ingestion of an HSD results in decreased insulin production (reduced Dilp2,5) and increased insulin resistance, indicating a type 2 diabetes phenotype $[135,142,230]$. These findings are in accordance with the results from mammals, in which hyperglycemia initially leads to increased insulin secretion and, over time, decreased insulin secretion by pancreatic $\beta$-cells [231,232].

Obesity per se and associated pathologies such as diabetes or hypertension are linked with diseases of the cardiovascular system. D. melanogaster and mammals exhibit many similarities in the cardiovascular system, such as a four-chambered heart structure [233], cardiac contraction-dependent transport, and similar cardiac output and flow rates in large vessels (e.g., aorta) [234], allowing, to a certain extent, for the application of the fruit fly for research on heart and circulatory diseases.

HSD and HFD intake are associated with increased cardiac fat storage, structural pathology such as collagen deposition, decreased contractility, conduction blocks, arrhythmia and myocardial enlargement in D. melanogaster. These pathological changes in the heart are related to systemic dysregulations, for example, in the context of energy supply and utilization via insulin-TOR signaling and via hexosamine flux and autonomic changes in tissues (e.g., adipose tissue, heart tissue). A reduction in lifespan is also evident under these conditions $[22,135]$.

Compared to obesity in D. melanogaster, obesity in mammals is associated with myocardial fat deposition and fibrosis, diastolic and systolic dysfunction, arrhythmias, hypertension, heart failure and ischemic and hemorrhagic stroke, having a negative impact on life expectancy. However, an obesity paradox is described, in which obesity provides an advantage in a pre-existing pathology such as coronary artery disease and improves survival [235].

The obesity paradox, which suggests a beneficial effect of accumulated fat, also seems to be observable in the model organism, D. melanogaster. For example, fat droplets in the glial niche decrease ROS during oxidative stress and prevent the oxidation of PUFAs, protecting glial cells and neuroblasts [236].

However, in a clear difference from mammals, D. melanogaster exhibits an open circulatory system with hemolymph, as opposed to the mammalian, closed blood circulation. Thus, pathological abnormalities such as the deposition of atherosclerotic plaques cannot be studied in D. melanogaster.

Metabolic consequences of obesity can also involve the liver, which has led to increased incidences of human liver diseases worldwide, such as nonalcoholic fatty liver disease and hepatocellular carcinoma [237].

Invertebrates such as D. melanogaster demonstrate an evolutionarily conserved liver function, with the fat body and oenocytes exhibiting glycogen storage and lipid processing equivalent to the livers of vertebrates. In D. melanogaster, 22 genes of lipid metabolism orthologous to human genes are expressed in oenocytes. The lipid content in oenocytes is regulated by the lipase BRUMMER and LIPID STORAGE DROPLET-2 of the fat body, which are homologous to ATGL and perilipin (PLIN) in mammals [238].

The fruit fly reveals many concordant genetic and metabolic traits of liver diseases, which enables the investigation of modifying factors and pathogenesis. Metabolic disruption of lipid and glucose metabolism, for example, via the alteration of the expression of $d T O R, F A S N$, sterol regulatory element-binding protein (SREBP) and Dilps in response to an HSD and HFD, negatively affects tissues and organs [239]. The structure of the fat body and oenocytes can be visualized by staining to detect irregular arrangement, altered sizes or altered quantities of stored lipid droplets. HSD and HFD feeding increases the number and size of lipid droplets in the fat body, similar to the situation in mammalian 
steatosis $[23,129]$. These initial conditions can chronically lead to the development of liver inflammation, cirrhosis or carcinoma [239].

Furthermore, obesity and comorbidities (e.g., diseases, hypertension) are major risk factors for the development of kidney disease.

A function homologous to that of the mammalian kidneys is found in nephrocytes and Malpighian tubules in the model organism, D. melanogaster. Hemolymph is filtered in nephrocytes, and urine is released into the hindgut via the Malpighian tubules following the absorption of required substances such as water, iron and organic metabolites. Additionally, the molecular pathways are conserved in the fruit fly, which enables the investigation of genetic changes such as renal development, cell differentiation or renal pathologies [233,240].

HSD consumption results in metabolic dysfunctions in D. melanogaster that predispose the flies to insulin resistance and nephrocyte failure. In response to HSD feeding, the expression of sticks and stones (Sns), regulated by the hexosamine biosynthesis pathway and the Polycomb gene complex, declines, affecting the function of the immunoglobulin superfamily protein $\mathrm{Sns}$, a homolog of mammalian nephrin. Sns and Kirre, homologous to Neph1, constitute part of the nephrocytic diaphragm and are essential for the ultrafiltration of hemolymph in garland nephrocytes. Structural changes in nephrocytes decrease ultrafiltration, causing, for example, the development of insulin resistance and increased proteinuria, indicating diabetic nephropathy parallel to that in mammals [241,242].

Additionally, HSD consumption leads to morphological alterations in Malpighian tubules involving reduced anterior and posterior diameters and a decreased density of microvilli in the brush border, resulting in a reduction in fluid secretion rate. Oxidative stress is also elevated, and inflammatory processes are activated in Malpighian tubules, leading to renal tubular dysfunction [140]. In mammals, increased oxidative stress and inflammatory processes are similarly linked to the development of chronic kidney disease [243].

In addition, the pathology of nephrolithiasis can be studied in D. melanogaster. HSD consumption increases the uric acid concentration in the hemolymph and enhances acidification to increase the formation of uric acid stones via purine catabolism [137]. Similarly, an increased acid load in the kidneys (e.g., due to increased endogenous synthesis), the insufficient availability of $\mathrm{H}^{+}$buffers and a low urine $\mathrm{pH}$ promote the formation of renal stones in humans [244]. The formation of the most common type of calcium oxalate renal stones in humans [245] can also be induced in the fruit fly by direct administration of lithogenic substances, e.g., calcium oxalate or ethylene glycol, in the diet [246].

Moreover, obesity can negatively affect the reproductive capacity of males and females. At both the genetic and morphological levels, many similarities between $D$. melanogaster and mammals are present.

In male fruit flies, the main cell types (e.g., spermatogonial stem cells, spermatocytes, meiotic cells) and the steps of spermatogenesis in the testes are analogous to those in mammals [247]. Diet affects male fertility, where a high-carbohydrate and low-protein diet leads to improved short-term offspring productivity. Carbohydrates seem to maintain the energy supply level and ensure the production of sex peptides and ovulin in the seminal fluid, which affects reproductive success [248]. HFD feeding in males causes a downregulation of glycosidase (e.g., $\alpha$-mannosidase) activity at the transcriptional level, which could directly affect gametogenesis, and consequently, sperm quality and function [159]. Similarly, HFD feeding in male rats induces obesity and adversely affects carbohydrate metabolism and testicular morphology, disrupting spermatogenesis [249]. Additionally, in men, correlations among obesity, comorbidities such as diabetes and reduced fertility caused by, for example, erectile dysfunction, a reduced semen quality or increased germ cell apoptosis can be observed [250].

The ovaries in female $D$. melanogaster have a cellular organization similar to that in mammals. Each follicle comprises 15 nurse cells and one oocyte enclosed by follicle cells. The ovaries of both fruit flies and mammals contain follicles at different stages of development and maturation [251]. 
HSD-induced obesity reduces ovary size in D. melanogaster, with the increased storage of TAGs and cholesterol and reductions in mature egg numbers [142] and fecundity [75]. In addition, increased levels of oxidative stress and alterations of the mitochondrial gene expression profile are observed in the ovaries [142].

A similar observation was made in obese women exhibiting altered oocyte morphology with increased cytoplasmic granularity [252]. The oocyte size is also reduced, and the resultant blastocysts contain reduced numbers of cells and higher TAG content [253]. Additionally, increased incidences of menstrual abnormalities (e.g., irregularity), polycystic ovarian syndrome, and ovulatory dysfunction and reduced spontaneous pregnancy rates are observed [254]. These findings are coupled with increases in oxidative stress and inflammation in the ovaries [255].

\subsection{Lessons Learned from D. Melanogaster}

The previous paragraph shows that central aspects of the D. melanogaster lipid metabolism such as FA synthesis and lipolysis were found to be evolutionarily conserved when compared to mammalian organisms. This raises the question whether the powerful fruit fly model can be used to identify novel genes, uncover novel aspects of lipid metabolism and help to develop novel anti-adipose strategies and treatments. Being a genetic model in the first place, it is no wonder that, in recent years, several genes that are involved in the regulation of the lipid metabolism were identified by forward and reverse genetic approaches in the fruit fly model first, before they were validated in mammals. For example, a D. melanogaster adp (adipose) loss-of-function mutant exhibits increased fat storage [19]. Genetic analyses revealed that $a d p$ encodes a WD40/tetratricopeptide-repeat-domain protein (WDTC). The mammalian homolog WDTC1 is a component of a DDB1-CUL4-ROC1 (CRL4) E3 ligase and was later shown to suppress adipogenesis via the regulation of peroxisome, proliferator-activated receptor- $\gamma$ activity $[256,257]$. Remarkably, WDTC1 SNP variants were defined as human obesity markers [258].

Two large-scale RNAi screens revealed approximately 500 and 60 novel candidate obesity genes, respectively, of which a great proportion had a homolog in mammals $[259,260]$. Based on these results, subsequent mice studies unveiled the role of Hedgehog signaling in mammalian adipocyte differentiation [260] and the regulatory role of the universal, store-operated calcium entry pathway in lipid metabolism [261].

On the other hand, the D. melanogaster model was successfully used to verify the function of candidate obesity genes that were identified by human genome-wide association studies as loci associated with adiposity [262] and as rare variants by the exome sequencing of severely obese children [263]. Testing the physiological function of the homologous genes by RNAi in the fruit fly model led to the discovery of 26 and 4 genes, respectively, which had not previously been linked to human obesity [262,263].

Due to the abovementioned high degree of functional and/or evolutionary conservation of the nutritional physiology and the key pathways of energy metabolism, D. melanogaster is recognized as a screenable model for anti-obesity agents [264-267]. Although published in an unchronological order, the aqueous extract of the brown algae Saccorhiza polyschides was first identified for mitigating the delirious effects of HFD in D. melanogaster [267] before it was found to counteract diet-induced obesity in mice [268]. In a recent study, a hydroethanolic extract of Lampaya medicinalis was tested for anti-adipogenic effects in D. melanogaster and HepG2 hepatocytes, preventing HFD-induced TAG accumulation and an accompanied inflammatory response in both systems. Compared to in vitro screens, the use of the fruit fly has the advantage of covering pharmacological aspects, including bioavailability, the influence of the gut microbiota and biotransformation. Moreover, the employment of fruit flies allows a cheap and rapid in vivo evaluation without major ethical considerations. Hence, it has the potential to precede/replace rodent studies at early stages of compound screening/identification. Lastly, apart from diet-induced obesity models, drug screening is also feasible in a number of genetic obesity models. 


\section{Conclusions}

Overall, a variety of different methods, such as the determination of body composition, food intake and metabolic rate, enable comprehensive phenotyping of the model organism, D. melanogaster (Figure 16). The diversity of methodologies allows the adaptation to different research questions, laboratory equipment, and time and financial frameworks. The structures of many organs, such as the digestive tract, and metabolic signaling pathways, such as the regulation of energy homeostasis via peptide hormones, are evolutionary and/or functionally conserved in D. melanogaster, making the fruit fly a well-suited model organism to study chronic diseases such as obesity and diabetes in a nutritional context from phenotypes to underlying cellular and molecular mechanisms. Furthermore, the influence of the parental diet on offspring, such as those in the F1 or F2 generation, can be studied.

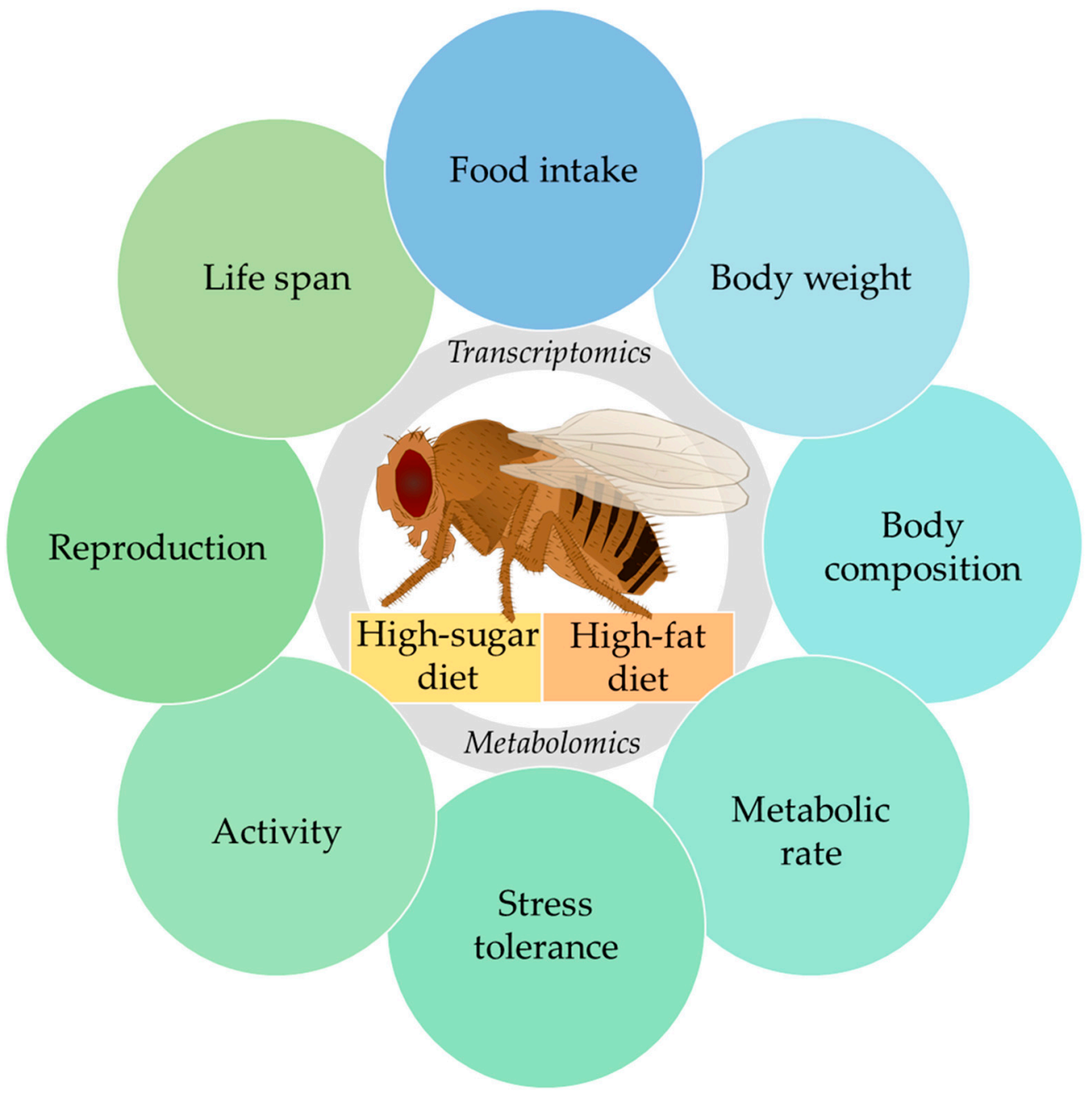

Figure 16. Overview of the methodological tool box that can be applied to study the responses of Drosophila melanogaster to high-sugar or high-fat diets from the molecular level to phenotypical traits.

Using D. melanogaster as a model organism has merits but also some limitations. First, not all organ and tissue structures of the fruit fly are functionally equivalent to those of humans and other mammalian species and some are even lacking. For instance, the fruit fly has only an innate immune system, which excludes studies of the response of the adaptive immune system to changing environmental factors such as microbial exposure.

In the future, there is a requirement for a standardization of dietary interventions for D. melanogaster. Terms such as "standard medium", "cultivation medium", "HSD" and "HFD" should be unified across laboratories in terms of the ingredients and their amounts. 
This will simplify the comparability of the study results of the different Drosophila research groups worldwide.

In summary, based on the diversity of methods for comprehensive phenotyping and its application in studies with dietary interventions, it can be concluded that D. melanogaster is a suitable and versatile model organism for the nutritional sciences.

Author Contributions: Conceptualization, V.E., K.L., S.S. and G.R.; methodology, V.E.; software, V.E.; validation, V.E., K.L., S.S. and G.R.; formal analysis, V.E.; investigation, V.E.; resources, V.E., K.L. and G.R.; data curation, V.E., K.L. and G.R.; writing-original draft preparation, V.E.; writing-review and editing, V.E., K.L., S.S. and G.R.; visualization, V.E.; supervision, K.L., S.S. and G.R.; project administration, K.L., S.S. and G.R.; funding acquisition, G.R. and S.S. All authors have read and agreed to the published version of the manuscript.

Funding: This work was funded by DFG, grant number 398035888. This review also received financial support for publication by Land Schleswig-Holstein within the funding program "Open Access-Publikationsfonds".

Institutional Review Board Statement: Not applicable.

Informed Consent Statement: Not applicable.

Data Availability Statement: Not applicable.

Acknowledgments: The authors acknowledge the financial support by DFG and the federal state of Schleswig-Holstein within the funding program "Open Access-Publikationsfonds".

Conflicts of Interest: The authors declare no conflict of interest.

\section{Abbreviations}

7-ADD

Acetyl-CoA

$4 \mathrm{EBP}$

AKHR

AKT

AMPK

ATGL

BCA

CAFE

CCA

Con-Ex

DAG

DAM

dFOXO

DHE

Dilp

EB, EC, EE

ERK

EX-Q

FA

FAS

FLIC

flyPAD

GABA

GFP

GO

HBP

HFD

HK

HSD

Hsl
7-Aminoactinomycin D

Acetyl-coenzyme A

eIF4E-binding protein

Adipokinetic hormone receptor

Protein kinase B

Adenosine monophosphate-activated protein kinase

Adipose triglyceride lipase

Bicinchoninic acid

CApillary FEeder

Coupled colorimetric assay

Consumption-excretion

Diacylglycerol

Drosophila Activity Monitoring

Forkhead Box O

Dihydroethidium

Insulin-like peptide

Enteroblast, enterocytes, enteroendocrine

Extracellular signal-regulated kinase

Excreta quantification

Fatty acid

Fatty acid synthase

Fly Liquid-Food Interaction Counter

Fly Proboscis and Activity Detector

Gamma-amino-butyric acid

Green fluorescent protein

Glucose oxidase

Hexosamine biosynthetic pathway

High-fat diet

Hexokinase

High-sugar diet

Hormone-sensitive lipase 


\begin{tabular}{|c|c|}
\hline ISC & Intestinal stem cell \\
\hline JAK & Janus kinase \\
\hline JNK & c-Jun $N$-terminal kinase \\
\hline $\mathrm{LC} / \mathrm{MS} ; \mathrm{GC} / \mathrm{MS}$ & Liquid chromatography or gas chromatography/mass spectrometry \\
\hline LSD & Low-sugar diet \\
\hline MAG & Monoacylglycerol \\
\hline MAFE & Manual feeding \\
\hline MARK & Mitogen-activated protein kinase \\
\hline NADH & Nicotinamide-adenine dinucleotide \\
\hline NADPH & Nicotinamide-adenine dinucleotide phosphate \\
\hline O-GlcNAc & O-linked $N$-acetylglucosamine \\
\hline OGT & O-GlcNAc transferase \\
\hline PAT & Perilipin, ADRP, TIP47 \\
\hline PBT & Dulbecco's phosphate-buffered saline with Triton X-100 \\
\hline PER & Proboscis extension response \\
\hline PI & Preference index \\
\hline PKA & Protein kinase A \\
\hline qRT-PCR & Real-time quantitative PCR \\
\hline RING & Rapid iterative negative geotaxis \\
\hline SAM (ELISA) & S-Adenosylmethionine ELISA \\
\hline SIK3 & Serine/threonine-protein kinase 3 \\
\hline STAT & Signal transducer and activator of transcription \\
\hline TAG & Triacylglyceride \\
\hline TCA & Tricarboxylic acid \\
\hline TLC & Thin-layer chromatography \\
\hline TOR & Target of rapamycin \\
\hline TUNEL & Terminal deoxynucleotidyl transferase dUTP nick end labeling \\
\hline VLDL & Very-low-density lipoprotein \\
\hline WDTC & WD40/tetratricopeptide-repeat-domain protein \\
\hline
\end{tabular}

\section{References}

1. Fernández-Moreno, M.A.; Farr, C.L.; Kaguni, L.S.; Garesse, R. Drosophila melanogaster as a Model System to Study Mitochondrial Biology. Methods Mol. Biol. 2007, 372, 33-49. [PubMed]

2. Adams, M.D. The Genome Sequence of Drosophila melanogaster. Science 2000, 287, 2185-2195. [CrossRef] [PubMed]

3. Bier, E. Drosophila, the golden bug, emerges as a tool for human genetics. Nat. Rev. Genet. 2005, 6, 9-23. [CrossRef] [PubMed]

4. Lüersen, K.; Röder, T.; Rimbach, G. Drosophila melanogaster in nutrition research-The importance of standardizing experimental diets. Genes Nutr. 2019, 14, 3737. [CrossRef] [PubMed]

5. Apidianakis, Y.; Rahme, L.G. Drosophila melanogaster as a model for human intestinal infection and pathology. Dis. Model. Mech. 2010, 4, 21-30. [CrossRef]

6. Graham, P.; Pick, L. Drosophila as a Model for Diabetes and Diseases of Insulin Resistance. In Fly Models of Human Diseases, 1st ed.; Pick, L., Ed.; Elsevier/Academic Press: Cambridge, MA, USA, 2017; pp. 397-419.

7. Kreipke, R.E.; Kwon, Y.V.; Shcherbata, H.R.; Ruohola-Baker, H. Drosophila melanogaster as a Model of Muscle Degeneration Disorders. In Fly Models of Human Diseases, 1st ed.; Pick, L., Ed.; Elsevier/Academic Press: Cambridge, MA, USA, $2017 ;$ pp. 83-109.

8. Kayashima, Y.; Murata, S.; Sato, M.; Matsuura, K.; Asanuma, T.; Chimoto, J.; Ishii, T.; Mochizuki, K.; Kumazawa, S.; Nakayama, T.; et al. Tea polyphenols ameliorate fat storage induced by high-fat diet in Drosophila melanogaster. Biochem. Biophys. Rep. 2015, 4, 417-424. [CrossRef]

9. Wagner, A.E.; Piegholdt, S.; Rabe, D.; Baenas, N.; Schloesser, A.; Eggersdorfer, M.; Stocker, A.; Rimbach, G. Epigallocatechin gallate affects glucose metabolism and increases fitness and lifespan in Drosophila melanogaster. Oncotarget 2015, 6, 30568-30578. [CrossRef]

10. Piegholdt, S.; Rimbach, G.; Wagner, A.E. The phytoestrogen prunetin affects body composition and improves fitness and lifespan in male Drosophila melanogaster. FASEB J. 2016, 30, 948-958. [CrossRef]

11. Staats, S.; Wagner, A.E.; Lüersen, K.; Künstner, A.; Meyer, T.; Kahns, A.K.; Derer, S.; Graspeuntner, S.; Rupp, J.; Busch, H.; et al Dietary ursolic acid improves health span and life span in male Drosophila melanogaster. BioFactors 2018, 45, 169-186. [CrossRef]

12. Staats, S.; Wagner, A.; Kowalewski, B.; Rieck, F.; Soukup, S.; Kulling, S.; Rimbach, G. Dietary Resveratrol Does Not Affect Life Span, Body Composition, Stress Response, and Longevity-Related Gene Expression in Drosophila melanogaster. Int. J. Mol. Sci. 2018, 19, 223. [CrossRef]

13. Piper, M.D.W.; Partridge, L. Protocols to Study Aging in Drosophila. Methods Mol. Biol. 2016, 1478, 291-302. [PubMed]

14. Staats, S.; Lüersen, K.; Wagner, A.E.; Rimbach, G. Drosophila melanogaster as a Versatile Model Organism in Food and Nutrition Research. J. Agric. Food Chem. 2018, 66, 3737-3753. [CrossRef] [PubMed] 
15. Jumbo-Lucioni, P.; Ayroles, J.F.; Chambers, M.M.; Jordan, K.W.; Leips, J.; Mackay, T.F.C.; de Luca, M. Systems genetics analysis of body weight and energy metabolism traits in Drosophila melanogaster. BMC Genom. 2010, 11, S12. [CrossRef]

16. Burggren, W.; Souder, B.M.; Ho, D.H. Metabolic rate and hypoxia tolerance are affected by group interactions and sex in the fruit fly (Drosophila melanogaster): New data and a literature survey. Biol. Open 2017, 23, 59. [CrossRef] [PubMed]

17. Hildebrandt, A.; Bickmeyer, I.; Kühnlein, R.P. Reliable Drosophila body fat quantification by a coupled colorimetric assay. PLoS ONE 2011, 6, e23796. [CrossRef]

18. Wei, C.; Yan, Y.; Miao, X.; Jiao, R. Dissection and Lipid Droplet Staining of Oenocytes in Drosophila Larvae. J. Vis. Exp. 2019, e60606. [CrossRef]

19. Häder, T.; Müller, S.; Aguilera, M.; Eulenberg, K.G.; Steuernagel, A.; Ciossek, T.; Kühnlein, R.P.; Lemaire, L.; Fritsch, R.; Dohrmann, C.; et al. Control of triglyceride storage by a WD40/TPR-domain protein. EMBO Rep. 2003, 4, 511-516. [CrossRef]

20. Ugrankar, R.; Liu, Y.; Provaznik, J.; Schmitt, S.; Lehmann, M. Lipin is a central regulator of adipose tissue development and function in Drosophila melanogaster. Mol. Cell Biol. 2011, 31, 1646-1656. [CrossRef]

21. Grönke, S.; Beller, M.; Fellert, S.; Ramakrishnan, H.; Jäckle, H.; Kühnlein, R.P. Control of Fat Storage by a Drosophila PAT Domain Protein. Curr. Biol. 2003, 13, 603-606. [CrossRef]

22. Birse, R.T.; Choi, J.; Reardon, K.; Rodriguez, J.; Graham, S.; Diop, S.; Ocorr, K.; Bodmer, R.; Oldham, S. High-Fat-Diet-Induced Obesity and Heart Dysfunction Are Regulated by the TOR Pathway in Drosophila. Cell Metab. 2010, 12, 533-544. [CrossRef]

23. Diop, S.B.; Bisharat-Kernizan, J.; Birse, R.T.; Oldham, S.; Ocorr, K.; Bodmer, R. PGC-1/Spargel Counteracts High-Fat-Diet-Induced Obesity and Cardiac Lipotoxicity Downstream of TOR and Brummer ATGL Lipase. Cell Rep. 2015, 10, 1572-1584. [CrossRef]

24. Diop, S.; Birse, R.; Bodmer, R. High Fat Diet Feeding and High Throughput Triacylglyceride Assay in Drosophila Melanogaster. J. Vis. Exp. 2017, 127, 56029. [CrossRef] [PubMed]

25. McGowan, M.W.; Artiss, J.D.; Strandbergh, D.R.; Zak, B. A peroxidase-coupled method for the colorimetric determination of serum triglycerides. Clin. Chem. 1983, 29, 538-542. [CrossRef] [PubMed]

26. Fossati, P.; Prencipe, L. Serum triglycerides determined colorimetrically with an enzyme that produces hydrogen peroxide. Clin Chem. 1982, 28, 2077-2080. [CrossRef] [PubMed]

27. Al-Anzi, B.; Zinn, K. Colorimetric measurement of triglycerides cannot provide an accurate measure of stored fat content in Drosophila. PLoS ONE 2010, 5, e12353. [CrossRef]

28. Matsuda, H.; Yamada, T.; Yoshida, M.; Nishimura, T. Flies without trehalose. J. Biol. Chem. 2015, 290, 1244-1255. [CrossRef]

29. Bligh, E.G.; Dyer, W.J. A rapid method of total lipid extraction and purification. Can. J. Biochem. Physiol. 1959, 37, 911-917. [CrossRef]

30. Folch, J.; Lees, M.; Stanley, G.H. A simple method for the isolation and purification of total lipids from animal tissues. J. Biol. Chem. 1957, 226, 497-509. [CrossRef]

31. Al-Anzi, B.; Sapin, V.; Waters, C.; Zinn, K.; Wyman, R.J.; Benzer, S. Obesity-blocking neurons in Drosophila. Neuron 2009, 63, 329-341. [CrossRef]

32. Rietveld, A.; Neutz, S.; Simons, K.; Eaton, S. Association of sterol- and glycosylphosphatidylinositol-linked proteins with Drosophila raft lipid microdomains. J. Biol. Chem. 1999, 274, 12049-12054. [CrossRef]

33. Hammad, L.A.; Cooper, B.S.; Fisher, N.P.; Montooth, K.L.; Karty, J.A. Profiling and quantification of Drosophila melanogaster lipids using liquid chromatography/mass spectrometry. Rapid Commun. Mass Spectrom. 2011, 25, 2959-2968. [CrossRef] [PubMed]

34. Fan, W.; Lam, S.M.; Xin, J.; Yang, X.; Liu, Z.; Liu, Y.; Wang, Y.; Shui, G.; Huang, X. Drosophila TRF2 and TAF9 regulate lipid droplet size and phospholipid fatty acid composition. PLoS Genet. 2017, 13, e1006664. [CrossRef] [PubMed]

35. Köhler, K.; Brunner, E.; Guan, X.L.; Boucke, K.; Greber, U.F.; Mohanty, S.; Barth, J.M.I.; Wenk, M.R.; Hafen, E. A combined proteomic and genetic analysis identifies a role for the lipid desaturase Desat1 in starvation-induced autophagy in Drosophila. Autophagy 2009, 5, 980-990. [CrossRef] [PubMed]

36. Bradford, M.M. A rapid and sensitive method for the quantitation of microgram quantities of protein utilizing the principle of protein-dye binding. Anal. Biochem. 1976, 72, 248-254. [CrossRef]

37. Krohn, R.I. The colorimetric detection and quantitation of total protein. Curr. Protoc. Cell Biol. 2011, 23, A-31.

38. Smith, P.K.; Krohn, R.I.; Hermanson, G.T.; Mallia, A.K.; Gartner, F.H.; Provenzano, M.D.; Fujimoto, E.K.; Goeke, N.M.; Olson, B.J.; Klenk, D.C. Measurement of protein using bicinchoninic acid. Anal. Biochem. 1985, 150, 76-85. [CrossRef]

39. Tennessen, J.M.; Barry, W.E.; Cox, J.; Thummel, C.S. Methods for studying metabolism in Drosophila. Methods 2014, 68, 105-115. [CrossRef]

40. Haselton, A.T.; Fridell, Y.-W.C. Insulin injection and hemolymph extraction to measure insulin sensitivity in adult Drosophila melanogaster. J. Vis. Exp. 2011, e2722. [CrossRef]

41. Trinder, P. Determination of blood glucose using an oxidase-peroxidase system with a non-carcinogenic chromogen. J. Clin. Pathol. 1969, 22, 158-161. [CrossRef]

42. Galant, A.L.; Kaufman, R.C.; Wilson, J.D. Glucose: Detection and analysis. Food Chem. 2015, 188, 149-160. [CrossRef]

43. Yatsenko, A.S.; Marrone, A.K.; Kucherenko, M.M.; Shcherbata, H.R. Measurement of Metabolic Rate in Drosophila using Respirometry. J. Vis. Exp. 2014, e51681. [CrossRef] [PubMed]

44. Tremblay, M.; Rundle, H.D.; Videlier, M.; Careau, V.; Herberstein, M. Territoriality in Drosophila: Indirect effects and covariance with body mass and metabolic rate. Behav. Ecol. 2021, 102, 591. [CrossRef] 
45. Fiorino, A.; Thompson, D.; Yadlapalli, S.; Jiang, C.; Shafer, O.T.; Reddy, P.; Meyhofer, E. Parallelized, real-time, metabolic-rate measurements from individual Drosophila. Sci. Rep. 2018, 8, 14452. [CrossRef] [PubMed]

46. Chakraborty, T.S.; Gendron, C.M.; Lyu, Y.; Munneke, A.S.; DeMarco, M.N.; Hoisington, Z.W.; Pletcher, S.D. Sensory perception of dead conspecifics induces aversive cues and modulates lifespan through serotonin in Drosophila. Nat Commun. 2019, 10, 804. [CrossRef] [PubMed]

47. Walsberg, G.E.; Hoffman, T.C.M. Direct calorimetry reveals large errors in respirometric estimates of energy expenditure. J. Exp. Biol. 2005, 208, 1035-1043. [CrossRef]

48. Van Voorhies, W.A.; Khazaeli, A.A.; Curtsinger, J.W. Selected contribution: Long-lived Drosophila melanogaster lines exhibit normal metabolic rates. J. Appl. Physiol. 2003, 95, 2605-2613, discussion 2604. [CrossRef]

49. Levine, J.A. Measurement of energy expenditure. Public Health Nutr. 2005, 8, 1123-1132. [CrossRef]

50. Orgad, S.; Nelson, H.; Segal, D.; Nelson, N. Metal ions suppress the abnormal taste behavior of the Drosophila mutant malvolio. J. Exp. Biol. 1998, 201, 115-120. [CrossRef]

51. Bahadorani, S.; Bahadorani, P.; Phillips, J.P.; Hilliker, A.J. The effects of vitamin supplementation on Drosophila life span under normoxia and under oxidative stress. J. Gerontol. A Biol. Sci. Med. Sci. 2008, 63, 35-42. [CrossRef]

52. Bahadorani, S.; Hilliker, A.J. Cocoa confers life span extension in Drosophila melanogaster. Nutr. Res. 2008, 28, 377-382. [CrossRef]

53. Peng, C.; Chan, H.Y.E.; Huang, Y.; Yu, H.; Chen, Z.-Y. Apple polyphenols extend the mean lifespan of Drosophila melanogaster. J. Agric. Food Chem. 2011, 59, 2097-2106. [CrossRef] [PubMed]

54. Wong, R.; Piper, M.D.W.; Blanc, E.; Partridge, L. Pitfalls of measuring feeding rate in the fruit fly Drosophila melanogaster. Nat. Methods 2008, 5, 214-215. [CrossRef] [PubMed]

55. Shell, B.C.; Schmitt, R.E.; Lee, K.M.; Johnson, J.C.; Chung, B.Y.; Pletcher, S.D.; Grotewiel, M. Measurement of solid food intake in Drosophila via consumption-excretion of a dye tracer. Sci. Rep. 2018, 8, 11536. [CrossRef] [PubMed]

56. Wu, Q.; Yu, G.; Park, S.J.; Gao, Y.; Ja, W.W.; Yang, M. Excreta Quantification (EX-Q) for Longitudinal Measurements of Food Intake in Drosophila. iScience 2020, 23, 100776. [CrossRef]

57. Geer, B.W.; Olander, R.M.; Sharp, P.L. Quantification of dietary choline utilization in adult Drosophila melanogaster by radioisotope methods. J. Insect Physiol. 1970, 16, 33-43. [CrossRef]

58. Ayaki, T.; Ohshima, K.; Okumura, Y.; Yoshikawa, I.; Shiomi, T. The relationship between lethal mutation yield and intake of ethylnitrosourea (ENU) in Drosophila melanogaster. Environ. Mol. Mutagen. 1984, 6, 483-488. [CrossRef]

59. Thompson, E.D.; Reeder, B.A. Method for selecting exposure levels for the Drosophila sex-linked recessive lethal assay. Environ. Mol. Mutagen. 1987, 10, 357-365. [CrossRef]

60. Thompson, E.D.; Reeder, B.A.; Bruce, R.D. Characterization of a method for quantitating food consumption for mutation assays in Drosophila. Environ. Mol. Mutagen. 1991, 18, 14-21. [CrossRef]

61. Brummel, T.; Ching, A.; Seroude, L.; Simon, A.F.; Benzer, S. Drosophila lifespan enhancement by exogenous bacteria. Proc. Natl. Acad. Sci. USA 2004, 101, 12974-12979. [CrossRef]

62. Carvalho, G.B.; Kapahi, P.; Benzer, S. Compensatory ingestion upon dietary restriction in Drosophila melanogaster. Nat. Methods 2005, 2, 813-815. [CrossRef]

63. Ja, W.W.; Carvalho, G.B.; Zid, B.M.; Mak, E.M.; Brummel, T.; Benzer, S. Water- and nutrient-dependent effects of dietary restriction on Drosophila lifespan. Proc. Natl. Acad. Sci. USA 2009, 106, 18633-18637. [CrossRef] [PubMed]

64. Deshpande, S.A.; Carvalho, G.B.; Amador, A.; Phillips, A.M.; Hoxha, S.; Lizotte, K.J.; Ja, W.W. Quantifying Drosophila food intake: Comparative analysis of current methodology. Nat. Methods 2014, 11, 535-540. [CrossRef] [PubMed]

65. Ja, W.W.; Carvalho, G.B.; Mak, E.M.; Noelle, N.; Fang, A.Y.; Liong, J.C.; Brummel, T.; Benzer, S. Prandiology of Drosophila and the CAFE assay. Proc. Natl. Acad. Sci. USA 2007, 104, 8253-8256. [CrossRef] [PubMed]

66. Diegelmann, S.; Jansen, A.; Jois, S.; Kastenholz, K.; Velo Escarcena, L.; Strudthoff, N.; Scholz, H. The CApillary FEeder Assay Measures Food Intake in Drosophila melanogaster. J. Vis. Exp. 2017, 121, 55024. [CrossRef] [PubMed]

67. Garlapow, M.E.; Huang, W.; Yarboro, M.T.; Peterson, K.R.; Mackay, T.F.C.; Ko, D.C. Quantitative Genetics of Food Intake in Drosophila melanogaster. PLoS ONE 2015, 10, e0138129. [CrossRef]

68. Qi, W.; Yang, Z.; Lin, Z.; Park, J.-Y.; Suh, G.S.B.; Wang, L. A quantitative feeding assay in adult Drosophila reveals rapid modulation of food ingestion by its nutritional value. Mol. Brain 2015, 8, 87. [CrossRef]

69. Shiraiwa, T.; Carlson, J.R. Proboscis extension response (PER) assay in Drosophila. J. Vis. Exp. 2007, 193. [CrossRef]

70. Itskov, P.M.; Moreira, J.-M.; Vinnik, E.; Lopes, G.; Safarik, S.; Dickinson, M.H.; Ribeiro, C. Automated monitoring and quantitative analysis of feeding behaviour in Drosophila. Nat. Commun. 2014, 5, 4560. [CrossRef]

71. Ro, J.; Harvanek, Z.M.; Pletcher, S.D. FLIC: High-throughput, continuous analysis of feeding behaviors in Drosophila. PLoS ONE 2014, 9, e101107. [CrossRef]

72. Linford, N.J.; Bilgir, C.; Ro, J.; Pletcher, S.D. Measurement of lifespan in Drosophila melanogaster. J. Vis. Exp. 2013, e50068. [CrossRef]

73. Sun, Y.; Yolitz, J.; Wang, C.; Spangler, E.; Zhan, M.; Zou, S. Aging Studies in Drosophila melanogaster. Methods Mol. Biol. 2013, 1048, 77-93. [PubMed]

74. Pletcher, S.D.; Curtsinger, J.W. The influence of environmentally induced heterogeneity on age-specific genetic variance for mortality rates. Genet. Res. 2000, 75, 321-329. [CrossRef] [PubMed] 
75. Skorupa, D.A.; Dervisefendic, A.; Zwiener, J.; Pletcher, S.D. Dietary composition specifies consumption, obesity, and lifespan in Drosophila melanogaster. Aging Cell 2008, 7, 478-490. [CrossRef] [PubMed]

76. Pletcher, S.D.; Stumpf, M.P.H. Population Genomics: Ageing by Association. Curr. Biol. 2002, 12, R328-R330. [CrossRef]

77. Nicolai, S.; Rossi, A.; Di Daniele, N.; Melino, G.; Annicchiarico-Petruzzelli, M.; Raschellà, G. DNA repair and aging: The impact of the p53 family. Aging 2015, 7, 1050-1065. [CrossRef]

78. Rogina, B.; Helfand, S.L. Sir2 mediates longevity in the fly through a pathway related to calorie restriction. Proc. Natl. Acad. Sci. USA 2004, 101, 15998-16003. [CrossRef]

79. Benzer, S. Behavioral mutants of Drosophila isolated by countercurrent distribution. Proc. Natl. Acad. Sci. USA 1967, 58, 1112-1119. [CrossRef]

80. Konopka, R.J.; Benzer, S. Clock Mutants of Drosophila melanogaster. Proc. Natl. Acad. Sci. USA 1971, 68, 2112-2116. [CrossRef]

81. Nichols, C.D.; Becnel, J.; Pandey, U.B. Methods to assay Drosophila behavior. J. Vis. Exp. 2012, e3795. [CrossRef]

82. Gargano, J.W.; Martin, I.; Bhandari, P.; Grotewiel, M.S. Rapid iterative negative geotaxis (RING): A new method for assessing age-related locomotor decline in Drosophila. Exp. Gerontol. 2005, 40, 386-395. [CrossRef]

83. Madabattula, S.; Strautman, J.; Bysice, A.; O'Sullivan, J.; Androschuk, A.; Rosenfelt, C.; Doucet, K. Quantitative Analysis of Climbing Defects in a Drosophila Model of Neurodegenerative Disorders. J. Vis. Exp. 2015, 52741. [CrossRef] [PubMed]

84. Scaplen, K.M.; Mei, N.J.; Bounds, H.A.; Song, S.L.; Azanchi, R.; Kaun, K.R. Automated real-time quantification of group locomotor activity in Drosophila melanogaster. Sci. Rep. 2019, 9, 2112. [CrossRef] [PubMed]

85. Maia Chagas, A.; Prieto-Godino, L.L.; Arrenberg, A.B.; Baden, T. The $€ 100$ lab: A 3D-printable open-source platform for fluorescence microscopy, optogenetics, and accurate temperature control during behaviour of zebrafish, Drosophila, and Caenorhabditis elegans. PLoS Biol. 2017, 15, e2002702. [CrossRef] [PubMed]

86. Wolf, F.W.; Rodan, A.R.; Tsai, L.T.-Y.; Heberlein, U. High-Resolution Analysis of Ethanol-Induced Locomotor Stimulation in Drosophila. J. Neurosci. 2002, 22, 11035-11044. [CrossRef]

87. Seong, K.-H.; Matsumura, T.; Shimada-Niwa, Y.; Niwa, R.; Kang, S. The Drosophila Individual Activity Monitoring and Detection System (DIAMonDS). elife 2020, 9, 1321. [CrossRef]

88. Donelson, N.; Kim, E.Z.; Slawson, J.B.; Vecsey, C.G.; Huber, R.; Griffith, L.C.; van Swinderen, B. High-Resolution Positional Tracking for Long-Term Analysis of Drosophila Sleep and Locomotion Using the "Tracker" Program. PLoS ONE 2012, 7, e37250. [CrossRef]

89. Pfeiffenberger, C.; Lear, B.C.; Keegan, K.P.; Allada, R. Locomotor Activity Level Monitoring Using the Drosophila Activity Monitoring (DAM) System. Cold Spring Harb. Protoc. 2010, 2010, 1238-1241. [CrossRef]

90. Vogler, G.; Ocorr, K. Visualizing the Beating Heart in Drosophila. J. Vis. Exp. 2009, e1425. [CrossRef]

91. Pitnick, S.; Markow, T.A.; Spicer, G.S. Delayed male maturity is a cost of producing large sperm in Drosophila. Proc. Natl. Acad. Sci. USA 1995, 92, 10614-10618. [CrossRef]

92. Klepsatel, P.; Gáliková, M.; Xu, Y.; Kühnlein, R.P. Thermal stress depletes energy reserves in Drosophila. Sci. Rep. 2016, 6, 229. [CrossRef]

93. Miller, P.B.; Obrik-Uloho, O.T.; Phan, M.H.; Medrano, C.L.; Renier, J.S.; Thayer, J.L.; Wiessner, G.; Bloch Qazi, M.C. The song of the old mother: Reproductive senescence in female drosophila. Fly (Austin) 2014, 8, 127-139. [CrossRef] [PubMed]

94. Buck Louis, G.M. Fecundity and Fertility: Overview of human fecundity and fertility. In Reproductive and Perinatal Epidemiology, 1st ed.; Buck Louis, G.M., Platt, R.W., Eds.; Oxford University Press Inc.: New York, NY, USA, 2011; pp. 6-8.

95. Gayathri, M.V.; Krishnamurthy, N.B. Studies on the toxicity of the mercurial fungicide Agallol 3 in Drosophila melanogaster. Environ. Res. 1981, 24, 89-95. [CrossRef]

96. Menon, A.; Varma, V.; Sharma, V.K. Rhythmic egg-laying behaviour in virgin females of fruit flies Drosophila melanogaster. Chronobiol. Int. 2014, 31, 433-441. [CrossRef] [PubMed]

97. Singh, K.; Kochar, E.; Prasad, N.G.; Schausberger, P. Egg Viability, Mating Frequency and Male Mating Ability Evolve in Populations of Drosophila melanogaster Selected for Resistance to Cold Shock. PLoS ONE 2015, 10, e0129992. [CrossRef]

98. Burke, M.K.; Barter, T.T.; Cabral, L.G.; Kezos, J.N.; Phillips, M.A.; Rutledge, G.A.; Phung, K.H.; Chen, R.H.; Nguyen, H.D.; Mueller, L.D.; et al. Rapid divergence and convergence of life-history in experimentally evolved Drosophila melanogaster. Evolution 2016, 70, 2085-2098. [CrossRef]

99. Ng'oma, E.; King, E.G.; Middleton, K.M. A model-based high throughput method for fecundity estimation in fruit fly studies. Fly (Austin) 2018, 12, 183-190. [CrossRef]

100. Novoseltsev, V.N.; Novoseltseva, J.A.; Yashin, A.I. What does a fly's individual fecundity pattern look like?: The dynamics of resource allocation in reproduction and ageing. Mech. Ageing Dev. 2003, 124, 605-617. [CrossRef]

101. Aruna, S.; Flores, H.A.; Barbash, D.A. Reduced Fertility of Drosophila melanogaster Hybrid male rescue (Hmr) Mutant Females Is Partially Complemented by Hmr Orthologs from Sibling Species. Genetics 2009, 181, 1437-1450. [CrossRef]

102. Fricke, C.; Bretman, A.; Chapman, T. Adult male nutrition and reproductive success in Drosophila melanogaster. Evolution 2008, 62, 3170-3177. [CrossRef]

103. Tan, C.K.W.; Pizzari, T.; Wigby, S. Parental age, gametic age, and inbreeding interact to modulate offspring viability in Drosophila melanogaster. Evolution 2013, 99, 3043-3051.

104. Flaven-Pouchon, J.; Garcia, T.; Abed-Vieillard, D.; Farine, J.-P.; Ferveur, J.-F.; Everaerts, C. Transient and permanent experience with fatty acids changes Drosophila melanogaster preference and fitness. PLoS ONE 2014, 9, e92352. [CrossRef] [PubMed] 
105. Chen, P.B.; Kim, J.H.; Kim, D.; Clark, J.M.; Park, Y. Conjugated Linoleic Acid Regulates Body Composition and Locomotor Activity in a Sex-Dependent Manner in Drosophila melanogaster. Lipids 2018, 53, 825-834. [CrossRef] [PubMed]

106. Zandawala, M.; Nguyen, T.; Balanyà Segura, M.; Johard, H.A.D.; Amcoff, M.; Wegener, C.; Paluzzi, J.-P.; Nässel, D.R.; Schoofs, L. A neuroendocrine pathway modulating osmotic stress in Drosophila. PLoS Genet. 2021, 17, e1009425. [CrossRef] [PubMed]

107. Dillon, M.E.; Wang, G.; Garrity, P.A.; Huey, R.B. Thermal preference in Drosophila. J. Therm. Biol. 2009, 34, 109-119. [CrossRef]

108. Hercus, M.J. Lifespan extension of Drosophila melanogaster through hormesis by repeated mild heat stress. Biogerontology 2003, 4, 149-156. [CrossRef]

109. Bettencourt, B.R.; Hogan, C.C.; Nimali, M.; Drohan, B.W. Inducible and constitutive heat shock gene expression responds to modification of Hsp70 copy number in Drosophila melanogaster but does not compensate for loss of thermotolerance in Hsp70null flies. BMC Biol. 2008, 6, 155. [CrossRef]

110. Heinrichsen, E.T.; Haddad, G.G.; Missirlis, F. Role of High-Fat Diet in Stress Response of Drosophila. PLoS ONE 2012, 7, e42587. [CrossRef]

111. Andersen, J.L.; Manenti, T.; Sørensen, J.G.; MacMillan, H.A.; Loeschcke, V.; Overgaard, J.; Woods, A. How to assess Drosophila cold tolerance: Chill coma temperature and lower lethal temperature are the best predictors of cold distribution limits. Funct. Ecol. 2014, 29, 55-65. [CrossRef]

112. Livingston, D.B.H.; Patel, H.; Donini, A.; MacMillan, H.A. Active transport of brilliant blue FCF across the Drosophila midgut and Malpighian tubule epithelia. Comp. Biochem. Physiol. A Mol. Integr. Physiol. 2020, 239, 110588. [CrossRef]

113. Al-Momani, F.A.; Massadeh, A.M. Effect of Different Heavy-Metal Concentrations on Drosophila melanogaster Larval Growth and Development. Biol. Trace Elem. Res. 2005, 108, 271-278. [CrossRef]

114. Bahadorani, S.; Hilliker, A.J. Biological and Behavioral Effects of Heavy Metals in Drosophila melanogaster Adults and Larvae. J. Insect Behav. 2009, 22, 399-411. [CrossRef]

115. Tettweiler, G. Starvation and oxidative stress resistance in Drosophila are mediated through the eIF4E-binding protein, d4E-BP. Genes Dev. 2005, 19, 1840-1843. [CrossRef] [PubMed]

116. Ahmad, M.; Chaudhary, S.U.; Afzal, A.J.; Tariq, M. Starvation-Induced Dietary Behaviour in Drosophila melanogaster Larvae and Adults. Sci. Rep. 2015, 5, 956. [CrossRef] [PubMed]

117. Krupp, J.J.; Nayal, K.; Wong, A.; Millar, J.G.; Levine, J.D. Desiccation resistance is an adaptive life-history trait dependent upon cuticular hydrocarbons, and influenced by mating status and temperature in D. melanogaster. J. Insect Physiol. 2020, 121, 103990. [CrossRef] [PubMed]

118. Kaneuchi, T. Efficient measurement of $\mathrm{H} 2 \mathrm{O} 2$ resistance in Drosophila using an activity monitor. Biogerontology 2003, 4, 157-165. [CrossRef]

119. Jünger, M.A.; Rintelen, F.; Stocker, H.; Wasserman, J.D.; Végh, M.; Radimerski, T.; Greenberg, M.E.; Hafen, E. The Drosophila Forkhead Transcription Factor FOXO Mediates the Reduction in Cell Number Associated with Reduced Insulin Signaling. J. Biol. 2003, 2, 20. [CrossRef] [PubMed]

120. Rzezniczak, T.Z.; Douglas, L.A.; Watterson, J.H.; Merritt, T.J.S. Paraquat administration in Drosophila for use in metabolic studies of oxidative stress. Anal. Biochem. 2011, 419, 345-347. [CrossRef]

121. Lemaitre, B.; Reichhart, J.-M.; Hoffmann, J.A. Drosophila host defense: Differential induction of antimicrobial peptide genes after infection by various classes of microorganisms. Proc. Natl. Acad. Sci. USA 1997, 94, 14614-14619. [CrossRef]

122. Ambrose, R.L.; Lander, G.C.; Maaty, W.S.; Bothner, B.; Johnson, J.E.; Johnson, K.N. Drosophila A virus is an unusual RNA virus with a $\mathrm{T}=3$ icosahedral core and permuted RNA-dependent RNA polymerase. J. Gen. Virol. 2009, 90, 2191-2200. [CrossRef]

123. Siva-Jothy, J.A.; Prakash, A.; Vasanthakrishnan, R.B.; Monteith, K.M.; Vale, P.F. Oral Bacterial Infection and Shedding in Drosophila melanogaster. J. Vis. Exp. 2018, 57676. [CrossRef]

124. Woodcock, K.J.; Kierdorf, K.; Pouchelon, C.A.; Vivancos, V.; Dionne, M.S.; Geissmann, F. Macrophage-derived upd3 cytokine causes impaired glucose homeostasis and reduced lifespan in Drosophila fed a lipid-rich diet. Immunity 2015, 42, 133-144. [CrossRef] [PubMed]

125. Hariri, N.; Thibault, L. High-fat diet-induced obesity in animal models. Nutr. Res. Rev. 2010, 23, 270-299. [CrossRef]

126. Gáliková, M.; Klepsatel, P. Obesity and Aging in the Drosophila Model. Int. J. Mol. Sci. 2018, 19, 1896. [CrossRef] [PubMed]

127. Raubenheimer, D.; Machovsky-Capuska, G.E.; Chapman, C.A.; Rothman, J.M. Geometry of nutrition in field studies: An illustration using wild primates. Oecologia 2015, 177, 223-234. [CrossRef] [PubMed]

128. Buescher, J.L.; Musselman, L.P.; Wilson, C.A.; Lang, T.; Keleher, M.; Baranski, T.J.; Duncan, J.G. Evidence for transgenerational metabolic programming in Drosophila. Dis. Model. Mech. 2013, 6, 1123-1132. [CrossRef] [PubMed]

129. Palanker Musselman, L.; Fink, J.L.; Narzinski, K.; Ramachandran, P.V.; Sukumar Hathiramani, S.; Cagan, R.L.; Baranski, T.J. A high-sugar diet produces obesity and insulin resistance in wild-type Drosophila. Dis. Model. Mech. 2011, 4, 842-849. [CrossRef]

130. Colinet, H.; Larvor, V.; Bical, R.; Renault, D. Dietary sugars affect cold tolerance of Drosophila melanogaster. Metabolomics 2013, 9 , 608-622. [CrossRef]

131. May, C.E.; Vaziri, A.; Lin, Y.Q.; Grushko, O.; Khabiri, M.; Wang, Q.-P.; Holme, K.J.; Pletcher, S.D.; Freddolino, P.L.; Neely, G.G.; et al. High Dietary Sugar Reshapes Sweet Taste to Promote Feeding Behavior in Drosophila melanogaster. Cell Rep. 2019, 27, 1675-1685.e7. [CrossRef] 
132. Wilinski, D.; Winzeler, J.; Duren, W.; Persons, J.L.; Holme, K.J.; Mosquera, J.; Khabiri, M.; Kinchen, J.M.; Freddolino, P.L.; Karnovsky, A.; et al. Rapid metabolic shifts occur during the transition between hunger and satiety in Drosophila melanogaster. Nat. Commun. 2019, 10, 4052. [CrossRef]

133. Yu, S.; Zhang, G.; Jin, L.H. A high-sugar diet affects cellular and humoral immune responses in Drosophila. Exp. Cell Res. 2018, 368, 215-224. [CrossRef]

134. Villanueva, J.E.; Livelo, C.; Trujillo, A.S.; Chandran, S.; Woodworth, B.; Andrade, L.; Le, H.D.; Manor, U.; Panda, S.; Melkani, G.C. Time-restricted feeding restores muscle function in Drosophila models of obesity and circadian-rhythm disruption. Nat. Commun. 2019, 10, 2700. [CrossRef] [PubMed]

135. Na, J.; Musselman, L.P.; Pendse, J.; Baranski, T.J.; Bodmer, R.; Ocorr, K.; Cagan, R.; Rulifson, E. A Drosophila Model of High Sugar Diet-Induced Cardiomyopathy. PLoS Genet. 2013, 9, e1003175. [CrossRef] [PubMed]

136. Dobson, A.J.; Ezcurra, M.; Flanagan, C.E.; Summerfield, A.C.; Piper, M.D.W.; Gems, D.; Alic, N. Nutritional Programming of Lifespan by FOXO Inhibition on Sugar-Rich Diets. Cell Rep. 2017, 18, 299-306. [CrossRef] [PubMed]

137. Van Dam, E.; van Leeuwen, L.A.G.; dos Santos, E.; James, J.; Best, L.; Lennicke, C.; Vincent, A.J.; Marinos, G.; Foley, A.; Buricova, M.; et al. Sugar-Induced Obesity and Insulin Resistance Are Uncoupled from Shortened Survival in Drosophila. Cell Metab. 2020, 31, 710-725.e7. [CrossRef] [PubMed]

138. Zhang, X.; Jin, Q.; Jin, L.H. High sugar diet disrupts gut homeostasis though JNK and STAT pathways in Drosophila. Biochem. Biophys. Res. Commun. 2017, 487, 910-916. [CrossRef]

139. Pereira, M.T.; Malik, M.; Nostro, J.A.; Mahler, G.J.; Musselman, L.P. Effect of dietary additives on intestinal permeability in both Drosophila and a human cell co-culture. Dis. Model Mech. 2018, 11, dmm034520. [CrossRef] [PubMed]

140. Rani, L.; Saini, S.; Shukla, N.; Chowdhuri, D.K.; Gautam, N.K. High sucrose diet induces morphological, structural and functional impairments in the renal tubules of Drosophila melanogaster: A model for studying type-2 diabetes mediated renal tubular dysfunction. Insect Biochem. Mol. Biol. 2020, 125, 103441. [CrossRef]

141. Catalani, E.; Silvestri, F.; Bongiorni, S.; Taddei, A.R.; Fanelli, G.; Rinalducci, S.; de Palma, C.; Perrotta, C.; Prantera, G.; Cervia, D. Retinal damage in a new model of hyperglycemia induced by high-sucrose diets. Pharmacol. Res. 2021, 166, 105488. [CrossRef]

142. Brookheart, R.T.; Swearingen, A.R.; Collins, C.A.; Cline, L.M.; Duncan, J.G. High-sucrose-induced maternal obesity disrupts ovarian function and decreases fertility in Drosophila melanogaster. Biochim. Biophys. Acta Mol. Basis Dis. 2017, 1863, $1255-1263$. [CrossRef]

143. Öst, A.; Lempradl, A.; Casas, E.; Weigert, M.; Tiko, T.; Deniz, M.; Pantano, L.; Boenisch, U.; Itskov, P.M.; Stoeckius, M.; et al. Paternal Diet Defines Offspring Chromatin State and Intergenerational Obesity. Cell 2014, 159, 1352-1364. [CrossRef] [PubMed]

144. Liao, S.; Amcoff, M.; Nässel, D.R. Impact of high-fat diet on lifespan, metabolism, fecundity and behavioral senescence in Drosophila. Insect Biochem. Mol. Biol. 2020, 133, 103495. [CrossRef] [PubMed]

145. Oldham, S. Obesity and nutrient sensing TOR pathway in flies and vertebrates: Functional conservation of genetic mechanisms. Trends Endocrinol. Metab. 2011, 22, 45-52. [CrossRef] [PubMed]

146. Jung, J.; Kim, D.-I.; Han, G.-Y.; Kwon, H. The Effects of High Fat Diet-Induced Stress on Olfactory Sensitivity, Behaviors, and Transcriptional Profiling in Drosophila melanogaster. Int. J. Mol. Sci. 2018, 19, 2855. [CrossRef] [PubMed]

147. Huang, R.; Song, T.; Su, H.; Lai, Z.; Qin, W.; Tian, Y.; Dong, X.; Wang, L. High-fat diet enhances starvation-induced hyperactivity via sensitizing hunger-sensing neurons in Drosophila. elife 2020, 9, e53103. [CrossRef]

148. Wen, D.-T.; Zheng, L.; Yang, F.; Li, H.-Z.; Hou, W.-Q. Endurance exercise prevents high-fat-diet induced heart and mobility premature aging and dsir2 expression decline in aging Drosophila. Oncotarget 2018, 9, 7298. [CrossRef]

149. Schultzhaus, J.N.; Bennett, C.J.; Iftikhar, H.; Yew, J.Y.; Mallett, J.; Carney, G.E. High fat diet alters Drosophila melanogaster sexual behavior and traits: Decreased attractiveness and changes in pheromone profiles. Sci. Rep. 2018, 8, 647. [CrossRef]

150. Nazario-Yepiz, N.O.; Loustalot-Laclette, M.R.; Carpinteyro-Ponce, J.; Abreu-Goodger, C.; Markow, T.A.; Missirlis, F. Transcriptional responses of ecologically diverse Drosophila species to larval diets differing in relative sugar and protein ratios. PLoS ONE 2017, 12, e0183007. [CrossRef]

151. Loreto, J.S.; Ferreira, S.A.; Ardisson-Araújo, D.M.P.; Barbosa, N.V. Human type 2 diabetes mellitus-associated transcriptional disturbances in a high-sugar diet long-term exposed Drosophila melanogaster. Comp. Biochem. Physiol. Part D Genomics Proteomics 2021, 39, 100866. [CrossRef]

152. Hemphill, W.; Rivera, O.; Talbert, M. RNA-Sequencing of Drosophila melanogaster Head Tissue on High-Sugar and High-Fat Diets. G3 (Bethesda) 2018, 8, 279-290. [CrossRef]

153. Ng'oma, E.; Williams-Simon, P.A.; Rahman, A.; King, E.G. Diverse biological processes coordinate the transcriptional response to nutritional changes in a Drosophila melanogaster multiparent population. BMC Genom. 2020, 21, 686. [CrossRef]

154. Ye, J.; Cui, X.; Loraine, A.; Bynum, K.; Kim, N.C.; White, G.; De Luca, M.; Garfinkel, M.G.; Lu, X.; Ruden, D.M. Methods for nutrigenomics and longevity studies in Drosophila: Effects of diets high in sucrose, palmitic acid, soy, or beef. Methods Mol. Biol. 2007, 371, 111-141. [PubMed]

155. Ruden, D.M.; de Luca, M.; Garfinkel, M.D.; Bynum, K.L.; Lu, X. DROSOPHILA NUTRIGENOMICS CAN PROVIDE CLUES TO HUMAN GENE-NUTRIENT INTERACTIONS. Annu. Rev. Nutr. 2005, 25, 499-522. [CrossRef] [PubMed]

156. Heinrichsen, E.T.; Zhang, H.; Robinson, J.E.; Ngo, J.; Diop, S.; Bodmer, R.; Joiner, W.J.; Metallo, C.M.; Haddad, G.G. Metabolic and transcriptional response to a high-fat diet in Drosophila melanogaster. Mol. Metab. 2014, 3, 42-54. [CrossRef] [PubMed] 
157. Rivera, O.; McHan, L.; Konadu, B.; Patel, S.; Sint Jago, S.; Talbert, M.E. A high-fat diet impacts memory and gene expression of the head in mated female Drosophila melanogaster. J. Comp. Physiol. B 2019, 189, 179-198. [CrossRef] [PubMed]

158. Grönke, S.M. Identification and characterization of genes controlling energy homeostasis in Drosophila melanogaster. Ph.D. Dissertation, Technischen Universität Carolo-Wilhelmina, Braunschweig, Germany, 2005.

159. Stobdan, T.; Sahoo, D.; Azad, P.; Hartley, I.; Heinrichsen, E.; Zhou, D.; Haddad, G.G.; Skoulakis, E.M.C. High fat diet induces sex-specific differential gene expression in Drosophila melanogaster. PLoS ONE 2019, 14, e0213474. [CrossRef]

160. Fabian, L.; Brill, J.A. Drosophila spermiogenesis. Spermatogenesis 2014, 2, 197-212. [CrossRef]

161. Ekengren, S.; Hultmark, D. A Family of Turandot-Related Genes in the Humoral Stress Response of Drosophila. Biochem. Biophys. Res. Commun. 2001, 284, 998-1003. [CrossRef]

162. Azuma, M.; Fat Le, T.; Yoshimoto, Y.; Hiraki, N.; Yamanaka, M.; Omura, F.; Inoue, Y.H. RNA-seq analysis of diet-driven obesity and anti-obesity effects of quercetin glucoside or epigallocatechin gallate in Drosophila adults. Eur. Rev. Med. Pharmacol. Sci. 2019, 23, 857-876

163. Reed, L.K.; Lee, K.; Zhang, Z.; Rashid, L.; Poe, A.; Hsieh, B.; Deighton, N.; Glassbrook, N.; Bodmer, R.; Gibson, G. Systems Genomics of Metabolic Phenotypes in Wild-Type Drosophila melanogaster. Genetics 2014, 197, 781-793. [CrossRef]

164. Williams, S.; Dew-Budd, K.; Davis, K.; Anderson, J.; Bishop, R.; Freeman, K.; Davis, D.; Bray, K.; Perkins, L.; Hubickey, J.; et al. Metabolomic and Gene Expression Profiles Exhibit Modular Genetic and Dietary Structure Linking Metabolic Syndrome Phenotypes in Drosophila. G3 (Bethesda) 2015, 5, 2817-2829. [CrossRef]

165. Enell, L.E.; Kapan, N.; Söderberg, J.A.E.; Kahsai, L.; Nässel, D.R.; Bergmann, A. Insulin Signaling, Lifespan and Stress Resistance Are Modulated by Metabotropic GABA Receptors on Insulin Producing Cells in the Brain of Drosophila. PLoS ONE 2010, 5 , e15780. [CrossRef] [PubMed]

166. Simard, C.J.; Touaibia, M.; Allain, E.P.; Hebert-Chatelain, E.; Pichaud, N. Role of the Mitochondrial Pyruvate Carrier in the Occurrence of Metabolic Inflexibility in Drosophila melanogaster Exposed to Dietary Sucrose. Metabolites 2020, 10, 411. [CrossRef] [PubMed]

167. Bricker, D.K.; Taylor, E.B.; Schell, J.C.; Orsak, T.; Boutron, A.; Chen, Y.-C.; Cox, J.E.; Cardon, C.M.; van Vranken, J.G.; Dephoure, N.; et al. A Mitochondrial Pyruvate Carrier Required for Pyruvate Uptake in Yeast, Drosophila, and Humans. Science 2012, 337, 96-100. [CrossRef]

168. Gillette, C.M.; Hazegh, K.E.; Nemkov, T.; Stefanoni, D.; D’Alessandro, A.; Taliaferro, J.M.; Reis, T. Gene-Diet Interactions: Dietary Rescue of Metabolic Defects in spen-Depleted Drosophila melanogaster. Genetics 2020, 214, 961-975. [CrossRef]

169. Tuthill, B.F.; Searcy, L.A.; Yost, R.A.; Musselman, L.P. Tissue-specific analysis of lipid species in Drosophila during overnutrition by UHPLC-MS/MS and MALDI-MSI. J. Lipid Res. 2020, 61, 275-290. [CrossRef]

170. Hardy, C.M.; Birse, R.T.; Wolf, M.J.; Yu, L.; Bodmer, R.; Gibbs, A.G. Obesity-associated cardiac dysfunction in starvation-selected Drosophila melanogaster. Am. J. Physiol. Regul. Integr. Comp. Physiol. 2015, 309, R658-R667. [CrossRef] [PubMed]

171. Oza, V.; Aicher, J.; Reed, L. Random Forest Analysis of Untargeted Metabolomics Data Suggests Increased Use of Omega Fatty Acid Oxidation Pathway in Drosophila Melanogaster Larvae Fed a Medium Chain Fatty Acid Rich High-Fat Diet. Metabolites 2019, 9, 5. [CrossRef]

172. Cormier, R.J.; Strang, R.; Menail, H.; Touaibia, M.; Pichaud, N. Systemic and mitochondrial effects of metabolic inflexibility induced by high fat diet in Drosophila melanogaster. Insect Biochem. Mol. Biol. 2021, 133, 103556. [CrossRef]

173. Miguel-Aliaga, I.; Jasper, H.; Lemaitre, B. Anatomy and Physiology of the Digestive Tract of Drosophila melanogaster. Genetics 2018, 210, 357-396.

174. Horne, I.; Haritos, V.S.; Oakeshott, J.G. Comparative and functional genomics of lipases in holometabolous insects. Insect Biochem. Mol. Biol. 2009, 39, 547-567. [CrossRef]

175. Sieber, M.H.; Thummel, C.S. Coordination of Triacylglycerol and Cholesterol Homeostasis by DHR96 and the Drosophila LipA Homolog magro. Cell Metab. 2012, 15, 122-127. [CrossRef] [PubMed]

176. Grönke, S.; Mildner, A.; Fellert, S.; Tennagels, N.; Petry, S.; Müller, G.; Jäckle, H.; Kühnlein, R.P. Brummer lipase is an evolutionary conserved fat storage regulator in Drosophila. Cell Metab. 2005, 1, 323-330. [CrossRef] [PubMed]

177. Chapman, R.F. The Insects: Structure and Function, 5th ed.; Chapman, R.F., Simpson, S.J., Douglas, A.E., Eds.; Cambridge University Press: Cambridge, UK, 2013.

178. Demignot, S.; Beilstein, F.; Morel, E. Triglyceride-rich lipoproteins and cytosolic lipid droplets in enterocytes: Key players in intestinal physiology and metabolic disorders. Biochimie 2014, 96, 48-55. [CrossRef]

179. Heier, C.; Kühnlein, R.P. Triacylglycerol Metabolism in Drosophila melanogaster. Genetics 2018, 210, 1163-1184. [CrossRef]

180. Voght, S.P.; Fluegel, M.L.; Andrews, L.A.; Pallanck, L.J. Drosophila NPC1b Promotes an Early Step in Sterol Absorption from the Midgut Epithelium. Cell Metab. 2007, 5, 195-205. [CrossRef] [PubMed]

181. Majerowicz, D.; Gondim, K. Insect Lipid Metabolism: Insights into Gene Expression Regulation: Recent Trends in Gene Expression. In Recent Trends in Gene Expression, 1st ed; Mandal, S.S., Ed.; Nova Science Publishers: New York, NY, USA, 2013 ; pp. 147-190.

182. Palm, W.; Sampaio, J.L.; Brankatschk, M.; Carvalho, M.; Mahmoud, A.; Shevchenko, A.; Eaton, S. Lipoproteins in Drosophila melanogaster-Assembly, Function, and Influence on Tissue Lipid Composition. PLoS Genet 2012, 8, e1002828. [CrossRef]

183. Gesta, S.; Tseng, Y.-H.; Kahn, C.R. Developmental Origin of Fat: Tracking Obesity to Its Source. Cell 2007, 131, 242-256. [CrossRef]

184. Ameer, F.; Scandiuzzi, L.; Hasnain, S.; Kalbacher, H.; Zaidi, N. De novo lipogenesis in health and disease. Metabolism 2014, 63, 895-902. [CrossRef] 
185. Biesalski, H.K.; Grimm, P.; Nowitzki-Grimm, S. Taschenatlas Ernährung, 8th ed.; Georg Thieme Verlag: Stuttgart, Germany, 2020.

186. Coleman, R.A.; Mashek, D.G. Mammalian Triacylglycerol Metabolism: Synthesis, Lipolysis, and Signaling. Chem. Rev. 2011, 111, 6359-6386. [CrossRef]

187. Bi, J.; Xiang, Y.; Chen, H.; Liu, Z.; Grönke, S.; Kühnlein, R.P.; Huang, X. Opposite and redundant roles of the two Drosophila perilipins in lipid mobilization. J. Cell Sci. 2012, 125, 3568-3577. [CrossRef]

188. Duncan, R.E.; Ahmadian, M.; Jaworski, K.; Sarkadi-Nagy, E.; Sul, H.S. Regulation of Lipolysis in Adipocytes. Annu. Rev. Nutr. 2007, 27, 79-101. [CrossRef] [PubMed]

189. Thaker, V.V. Genetic and epigenetic causes of obesity. Adolesc. Med. State Art Rev. 2017, 28, 379-405. [PubMed]

190. Grönke, S.; Müller, G.; Hirsch, J.; Fellert, S.; Andreou, A.; Haase, T.; Jäckle, H.; Kühnlein, R.P.; O’Rahilly, S. Dual Lipolytic Control of Body Fat Storage and Mobilization in Drosophila. PLoS Biol. 2007, 5, e137. [CrossRef] [PubMed]

191. Schreiber, R.; Xie, H.; Schweiger, M. Of mice and men: The physiological role of adipose triglyceride lipase (ATGL). Biochim. Biophys. Acta Mol. Cell Biol. Lipids 2019, 1864, 880-899. [CrossRef]

192. Guilleminault, L.; Williams, E.; Scott, H.; Berthon, B.; Jensen, M.; Wood, L. Diet and Asthma: Is It Time to Adapt Our Message? Nutrients 2017, 9, 1227. [CrossRef]

193. Hu, F.B. Dietary pattern analysis: A new direction in nutritional epidemiology. Curr. Opin. Lipidol. 2002, 13, 3-9. [CrossRef]

194. Roser, M.; Ritchie, H. Food Supply. Available online: https:/ / ourworldindata.org/food-supply (accessed on 1 November 2021).

195. Caballero, B. Humans against Obesity: Who Will Win? Adv. Nutr. 2019, 10, S4-S9. [CrossRef]

196. Kopp, W. How Western Diet And Lifestyle Drive The Pandemic Of Obesity And Civilization Diseases. Diabetes Metab. Syndr. Obes. 2019, 12, 2221-2236. [CrossRef]

197. Swinburn, B.A.; Caterson, I.; Seidell, J.C.; James, W.P. Diet, nutrition and the prevention of excess weight gain and obesity. Public Health Nutr. 2004, 7, 123-146.

198. NCD-RisC. Trends in adult body-mass index in 200 countries from 1975 to 2014: A pooled analysis of 1698 population-based measurement studies with $19 \cdot 2$ million participants. Lancet 2016, 387, 1377-1396. [CrossRef]

199. de Moura e Dias, M.; dos Reis, S.A.; da Conceição, L.L.; de Oliveira Sediyama, C.M.N.; Pereira, S.S.; de Oliveira, L.L.; do Carmo Gouveia Peluzio, M.; Martinez, J.A.; Milagro, F.I. Diet-induced obesity in animal models: Points to consider and influence on metabolic markers. Diabetol. Metab. Syndr. 2021, 13, 47. [CrossRef] [PubMed]

200. Bortolin, R.C.; Vargas, A.R.; Gasparotto, J.; Chaves, P.R.; Schnorr, C.E.; Martinello, K.B.; Silveira, A.K.; Rabelo, T.K.; Gelain, D.P.; Moreira, J.C.F. A new animal diet based on human Western diet is a robust diet-induced obesity model: Comparison to high-fat and cafeteria diets in term of metabolic and gut microbiota disruption. Int. J. Obes. 2018, 42, 525-534. [CrossRef] [PubMed]

201. Rothwell, N.J.; Stock, M.J. The development of obesity in animals: The role of dietary factors. Clin. Endocrinol. Metab. 1984, 13, 437-449. [CrossRef]

202. Murashov, A.K.; Pak, E.S.; Lin, C.-T.; Boykov, I.N.; Buddo, K.A.; Mar, J.; Bhat, K.M.; Neufer, P.D. Preference and detrimental effects of high fat, sugar, and salt diet in wild-caught Drosophila simulans are reversed by flight exercise. FASEB Bioadv. 2021, 3, 49-64. [CrossRef] [PubMed]

203. Drewnowski, A.; Kurth, C.; Holden-Wiltse, J.; Saari, J. Food preferences in human obesity: Carbohydrates versus fats. Appetite 1992, 18, 207-221. [CrossRef]

204. Lampuré, A.; Schlich, P.; Deglaire, A.; Castetbon, K.; Péneau, S.; Hercberg, S.; Méjean, C. Sociodemographic, Psychological, and Lifestyle Characteristics Are Associated with a Liking for Salty and Sweet Tastes in French Adults. Br. J. Nutr. 2014, 112, 1353-1363. [CrossRef] [PubMed]

205. Bartoshuk, L.M.; Duffy, V.B.; Hayes, J.E.; Moskowitz, H.R.; Snyder, D.J. Psychophysics of sweet and fat perception in obesity: Problems, solutions and new perspectives. Phil. Trans. R. Soc. B 2006, 361, 1137-1148. [CrossRef]

206. Proserpio, C.; Laureati, M.; Bertoli, S.; Battezzati, A.; Pagliarini, E. Determinants of Obesity in Italian Adults: The Role of Taste Sensitivity, Food Liking, and Food Neophobia. Chem. Senses 2015, 4, bjv072. [CrossRef]

207. Berthoud, H.-R.; Zheng, H. Modulation of taste responsiveness and food preference by obesity and weight loss. Physiol. Behav. 2012, 107, 527-532. [CrossRef]

208. Spinelli, S.; Monteleone, E. Food Preferences and Obesity. Endocrinol. Metab. 2021, 36, 209-219. [CrossRef]

209. Deutsche Gesellschaft für Ernährung; Österreichische Gesellschaft für Ernährung; Schweizerische Gesellschaft für Ernährungsforschung. D-A-CH-Referenzwerte für die Nährstoffzufuhr, 2nd ed.; Neuer Umschau Buchverl.: Neustadt an der Weinstraße, Germany, 2015.

210. HHS; USDA. Dietary Guidelines for Americans, 2020-2025: Make Every Bite Count with the Dietary Guidelines, 9th ed.; U.S. Department of Agriculture: Washington, DC, USA, 2020.

211. Wolfram, G.; Bechthold, A.; Boeing, H.; Ellinger, S.; Hauner, H.; Kroke, A.; Leschik-Bonnet, E.; Linseisen, J.; Lorkowski, S.; Schulze, M.; et al. Evidence-Based Guideline of the German Nutrition Society: Fat Intake and Prevention of Selected Nutrition-Related Diseases. Ann. Nutr. Metab. 2015, 67, 141-204. [CrossRef] [PubMed]

212. Shi, D.; Han, T.; Chu, X.; Lu, H.; Yang, X.; Zi, T.; Zhao, Y.; Wang, X.; Liu, Z.; Ruan, J.; et al. An isocaloric moderately high-fat diet extends lifespan in male rats and Drosophila. Cell Metab. 2021, 33, 581-597.e9. [CrossRef] [PubMed]

213. Li, W.; Wang, Z.; Syed, S.; Lyu, C.; Lincoln, S.; O’Nell, J.; Nguyen, A.D.; Feng, I.; Young, M.W. Chronic social isolation signals starvation and reduces sleep in Drosophila. Nature 2021, 597, 239-244. [CrossRef] [PubMed] 
214. Lebedev, A.A.; Moskalev, A.R.; Abrosimov, M.E.; Vetlugin, E.A.; Pshenichnaya, A.G.; Lebedev, V.A.; Evdokimova, N.R.; Bychkov, E.R.; Shabanov, P.D. Effect of neuropeptide Y antagonist BMS193885 on overeating and emotional responses induced by social isolation in rats. Rev. Clin. Pharm. Drug Ther. 2021, 19, 189-202. [CrossRef]

215. Anversa, R.G.; Campbell, E.J.; Ch'ng, S.S.; Gogos, A.; Lawrence, A.J.; Brown, R.M. A model of emotional stress-induced binge eating in female mice with no history of food restriction. Genes Brain Behav. 2019, 19, e12613. [CrossRef]

216. Dos Santos Quaresma, M.V.L.; Marques, C.G.; Magalhães, A.C.O.; dos Santos, R.V.T. Emotional eating, binge eating, physical inactivity, and vespertine chronotype are negative predictors of dietary practices during COVID-19 social isolation: A crosssectional study. Nutrition 2021, 90, 111223. [CrossRef]

217. Kalkan Uğurlu, Y.; Mataracı Değirmenci, D.; Durgun, H.; Gök Uğur, H. The examination of the relationship between nursing students' depression, anxiety and stress levels and restrictive, emotional, and external eating behaviors in COVID-19 social isolation process. Perspect. Psychiatr. Care 2021, 57, 507-516. [CrossRef]

218. Zhang, Q. Characterization of Circadian Feeding Rhythms in Drosophila Using the Fly Liquid-Food Interaction Counter (FLIC) Assay. Ph.D. Dissertation, University of Michigan, Ann Arbor, MI, USA, 2016.

219. Harbison, S.T.; Sehgal, A.; Louis, M. Energy Stores Are Not Altered by Long-Term Partial Sleep Deprivation in Drosophila melanogaster. PLoS ONE 2009, 4, e6211. [CrossRef]

220. Froy, O. Circadian Rhythms and Obesity in Mammals. ISRN Obes. 2012, 2012, 437198. [CrossRef]

221. Sherman, H.; Genzer, Y.; Cohen, R.; Chapnik, N.; Madar, Z.; Froy, O. Timed high-fat diet resets circadian metabolism and prevents obesity. FASEB J. 2012, 26, 3493-3502. [CrossRef]

222. Marcheva, B.; Ramsey, K.M.; Buhr, E.D.; Kobayashi, Y.; Su, H.; Ko, C.H.; Ivanova, G.; Omura, C.; Mo, S.; Vitaterna, M.H.; et al. Disruption of the clock components CLOCK and BMAL1 leads to hypoinsulinaemia and diabetes. Nature 2010, 466, 627-631. [CrossRef] [PubMed]

223. Ahmad, M.; He, L.; Perrimon, N. Regulation of insulin and adipokinetic hormone/glucagon production in flies. WIREs Dev Biol 2020, 9, 65. [CrossRef] [PubMed]

224. Nässel, D.R.; Kubrak, O.I.; Liu, Y.; Luo, J.; Lushchak, O.V. Factors that regulate insulin producing cells and their output in Drosophila. Front. Physiol. 2013, 4, 252. [CrossRef]

225. Claeys, I.; Simonet, G.; Poels, J.; van Loy, T.; Vercammen, L.; de Loof, A.; Vanden Broeck, J. Insulin-related peptides and their conserved signal transduction pathway. Peptides 2002, 23, 807-816. [CrossRef]

226. Blenis, J. Signal transduction via the MAP kinases: Proceed at your own RSK. Proc. Natl. Acad. Sci. USA 1993, 90, 5889-5892. [CrossRef] [PubMed]

227. Shepherd, P.R.; Withers, D.J.; Siddle, K. Phosphoinositide 3-kinase: The key switch mechanism in insulin signalling. Biochem. J. 1998, 333, 471-490. [CrossRef]

228. Rintelen, F.; Stocker, H.; Thomas, G.; Hafen, E. PDK1 regulates growth through Akt and S6K in Drosophila. Proc. Natl. Acad. Sci. USA 2001, 98, 15020-15025. [CrossRef]

229. Wessells, R.; Fitzgerald, E.; Piazza, N.; Ocorr, K.; Morley, S.; Davies, C.; Lim, H.-Y.; Elmén, L.; Hayes, M.; Oldham, S.; et al. d4eBP acts downstream of both dTOR and dFoxo to modulate cardiac functional aging in Drosophila. Aging Cell 2009, 8, 542-552. [CrossRef]

230. Morris, S.N.S.; Coogan, C.; Chamseddin, K.; Fernandez-Kim, S.O.; Kolli, S.; Keller, J.N.; Bauer, J.H. Development of diet-induced insulin resistance in adult Drosophila melanogaster. Biochim. Biophys. Acta 2012, 1822, 1230-1237. [CrossRef]

231. Reaven, G.M. Pathophysiology of insulin resistance in human disease. Physiol. Rev. 1995, 75, 473-486. [CrossRef]

232. Kahn, S.E.; Hull, R.L.; Utzschneider, K.M. Mechanisms linking obesity to insulin resistance and type 2 diabetes. Nature 2006, 444, 840-846. [CrossRef] [PubMed]

233. Ugur, B.; Chen, K.; Bellen, H.J. Drosophila tools and assays for the study of human diseases. Dis. Model. Mech. 2016, 9, 235-244. [CrossRef] [PubMed]

234. Choma, M.A.; Suter, M.J.; Vakoc, B.J.; Bouma, B.E.; Tearney, G.J. Physiological homology between Drosophila melanogaster and vertebrate cardiovascular systems. Dis. Model. Mech. 2011, 4, 411-420. [CrossRef] [PubMed]

235. Csige, I.; Ujvárosy, D.; Szabó, Z.; Lőrincz, I.; Paragh, G.; Harangi, M.; Somodi, S. The Impact of Obesity on the Cardiovascular System. J. Diabetes Res. 2018, 2018, 3407306. [CrossRef] [PubMed]

236. Bailey, A.P.; Koster, G.; Guillermier, C.; Hirst, E.M.A.; MacRae, J.I.; Lechene, C.P.; Postle, A.D.; Gould, A.P. Antioxidant Role for Lipid Droplets in a Stem Cell Niche of Drosophila. Cell 2015, 163, 340-353. [CrossRef]

237. Asrani, S.K.; Devarbhavi, H.; Eaton, J.; Kamath, P.S. Burden of liver diseases in the world. J. Hepatol. 2019, 70, 151-171. [CrossRef]

238. Gutierrez, E.; Wiggins, D.; Fielding, B.; Gould, A.P. Specialized hepatocyte-like cells regulate Drosophila lipid metabolism. Nature 2007, 445, 275-280. [CrossRef]

239. Moraes, K.C.M.; Montagne, J. Drosophila melanogaster: A Powerful Tiny Animal Model for the Study of Metabolic Hepatic Diseases. Front. Physiol. 2021, 12, 390. [CrossRef]

240. Allocca, M.; Zola, S.; Bellosta, P. The Fruit Fly, Drosophila melanogaster: Modeling of Human Diseases (Part II). In Drosophila melanogaster - Model for Recent Advances in Genetics and Therapeutics, 1st ed.; Perveen, F.K., Ed.; InTech: Rijeka, Croatia, 2018; pp. 138-139.

241. Na, J.; Sweetwyne, M.T.; Park, A.S.D.; Susztak, K.; Cagan, R.L. Diet-Induced Podocyte Dysfunction in Drosophila and Mammals. Cell Rep. 2015, 12, 636-647. [CrossRef] 
242. Zhuang, S.; Shao, H.; Guo, F.; Trimble, R.; Pearce, E.; Abmayr, S.M. Sns and Kirre, the Drosophila orthologs of Nephrin and Neph1, direct adhesion, fusion and formation of a slit diaphragm-like structure in insect nephrocytes. Development 2009, 136, 2335-2344. [CrossRef]

243. Kovesdy, C.P.; Furth, S.; Zoccali, C.; on Behalf of the World Kidney Day Steering Comittee. Obesity and kidney disease: Hidden consequences of the epidemic. Indian J. Nephrol. 2017, 27, 85. [CrossRef] [PubMed]

244. Wiederkehr, M.R.; Moe, O.W. Uric Acid Nephrolithiasis: A Systemic Metabolic Disorder. Clinic Rev. Bone Miner. Metab. 2011, 9 , 207-217. [CrossRef]

245. Poore, W.; Boyd, C.J.; Singh, N.P.; Wood, K.; Gower, B.; Assimos, D.G. Obesity and Its Impact on Kidney Stone Formation. Rev. Urol. 2020, 22, 17-23. [PubMed]

246. Chen, Y.-H.; Liu, H.-P.; Chen, H.-Y.; Tsai, F.-J.; Chang, C.-H.; Lee, Y.-J.; Lin, W.-Y.; Chen, W.-C. Ethylene glycol induces calcium oxalate crystal deposition in Malpighian tubules: A Drosophila model for nephrolithiasis/urolithiasis. Kidney Int. 2011, 80, 369-377. [CrossRef] [PubMed]

247. Bonilla, E.; Xu, E.Y. Identification and characterization of novel mammalian spermatogenic genes conserved from fly to human. Mol. Hum. Reprod. 2008, 14, 137-142. [CrossRef] [PubMed]

248. Morimoto, J.; Wigby, S. Differential effects of male nutrient balance on pre- and post-copulatory traits, and consequences for female reproduction in Drosophila melanogaster. Sci. Rep. 2016, 6, 1415. [CrossRef]

249. Campos-Silva, P.; Furriel, A.; Costa, W.S.; Sampaio, F.J.B.; Gregório, B.M. Metabolic and Testicular Effects of the Long-Term Administration of Different High-Fat Diets in Adult Rats. Int. Braz. J. Urol. 2015, 41, 569-575. [CrossRef]

250. Leisegang, K.; Sengupta, P.; Agarwal, A.; Henkel, R. Obesity and male infertility: Mechanisms and management. Andrologia 2021, 53, 2380. [CrossRef]

251. Armstrong, A.R. Drosophila melanogaster as a model for nutrient regulation of ovarian function. Reproduction 2020, 159, R69-R82. [CrossRef]

252. Depalo, R.; Garruti, G.; Totaro, I.; Panzarino, M.; Vacca, M.P.; Giorgino, F.; Selvaggi, L.E. Oocyte morphological abnormalities in overweight women undergoing in vitro fertilization cycles. Gynecol. Endocrinol. 2011, 27, 880-884. [CrossRef]

253. Leary, C.; Leese, H.J.; Sturmey, R.G. Human embryos from overweight and obese women display phenotypic and metabolic abnormalities. Hum. Reprod. 2014, 30, 122-132. [CrossRef] [PubMed]

254. Practice Committee of the American Society for Reproductive Medicine. Obesity and reproduction: A committee opinion. Fertil. Steril. 2015, 104, 1116-1126. [CrossRef] [PubMed]

255. Snider, A.P.; Wood, J.R. Obesity induces ovarian inflammation and reduces oocyte quality. Reproduction 2019, 158, R79-R90. [CrossRef] [PubMed]

256. Groh, B.S.; Yan, F.; Smith, M.D.; Yu, Y.; Chen, X.; Xiong, Y. The antiobesity factor WDTC 1 suppresses adipogenesis via the CRL 4 WDTC1 E3 ligase. EMBO Rep. 2016, 17, 638-647. [CrossRef]

257. Suh, J.M.; Zeve, D.; McKay, R.; Seo, J.; Salo, Z.; Li, R.; Wang, M.; Graff, J.M. Adipose Is a Conserved Dosage-Sensitive Antiobesity Gene. Cell Metab. 2007, 6, 195-207. [CrossRef]

258. Lai, C.-Q.; Parnell, L.D.; Arnett, D.K.; García-Bailo, B.; Tsai, M.Y.; Kabagambe, E.K.; Straka, R.J.; Province, M.A.; An, P.; Borecki, I.B.; et al. WDTC1, the Ortholog of Drosophila Adipose Gene, Associates With Human Obesity, Modulated by MUFA Intake. Obesity 2009, 17, 593-600. [CrossRef]

259. Baumbach, J.; Hummel, P.; Bickmeyer, I.; Kowalczyk, K.M.; Frank, M.; Knorr, K.; Hildebrandt, A.; Riedel, D.; Jäckle, H.; Kühnlein, R.P. A Drosophila In Vivo Screen Identifies Store-Operated Calcium Entry as a Key Regulator of Adiposity. Cell Metab. 2014, 19, 331-343. [CrossRef]

260. Pospisilik, J.A.; Schramek, D.; Schnidar, H.; Cronin, S.J.F.; Nehme, N.T.; Zhang, X.; Knauf, C.; Cani, P.D.; Aumayr, K.; Todoric, J.; et al. Drosophila genome-wide obesity screen reveals hedgehog as a determinant of brown versus white adipose cell fate. Cell 2010, 140, 148-160. [CrossRef]

261. Arruda, A.P.; Pers, B.M.; Parlakgul, G.; Güney, E.; Goh, T.; Cagampan, E.; Lee, G.Y.; Goncalves, R.L.; Hotamisligil, G.S. Defective STIM-mediated store operated Ca2+ entry in hepatocytes leads to metabolic dysfunction in obesity. elife 2017, 6, 14485. [CrossRef]

262. Baranski, T.J.; Kraja, A.T.; Fink, J.L.; Feitosa, M.; Lenzini, P.A.; Borecki, I.B.; Liu, C.-T.; Cupples, L.A.; North, K.E.; Province, M.A.; et al. A high throughput, functional screen of human Body Mass Index GWAS loci using tissue-specific RNAi Drosophila melanogaster crosses. PLoS Genet. 2018, 14, e1007222. [CrossRef]

263. Agrawal, N.; Lawler, K.; Davidson, C.M.; Keogh, J.M.; Legg, R.; Barroso, I.; Farooqi, I.S.; Brand, A.H.; Tapon, N. Predicting novel candidate human obesity genes and their site of action by systematic functional screening in Drosophila. PLoS Biol. 2021, 19, e3001255. [CrossRef] [PubMed]

264. Men, T.T.; van Thanh, D.N.; Yamaguchi, M.; Suzuki, T.; Hattori, G.; Arii, M.; Huy, N.T.; Kamei, K. A Drosophila Model for Screening Antiobesity Agents. Biomed Res. Int. 2016, 2016, 6293163. [CrossRef] [PubMed]

265. Sanhueza, S.; Tobar, N.; Cifuentes, M.; Quenti, D.; Varì, R.; Scazzocchio, B.; Masella, R.; Herrera, K.; Paredes, A.; Morales, G.; et al. Lampaya Medicinalis Phil. decreases lipid-induced triglyceride accumulation and proinflammatory markers in human hepatocytes and fat body of Drosophila melanogaster. Int. J. Obes. 2021, 45, 1464-1475. [CrossRef] [PubMed]

266. Günther, I.; Rimbach, G.; Nevermann, S.; Neuhauser, C.; Stadlbauer, V.; Schwarzinger, B.; Schwarzinger, C.; Ipharraguerre, I.R.; Weghuber, J.; Lüersen, K. Avens Root (Geum urbanum L.) Extract Discovered by Target-Based Screening Exhibits Antidiabetic Activity in the Hen's Egg Test Model and Drosophila melanogaster. Front. Pharmacol. 2021, 12, 55. [CrossRef] [PubMed] 
267. Li, Y.; Romey-Glüsing, R.; Tahan Zadeh, N.; von Frieling, J.; Hoffmann, J.; Huebbe, P.; Bruchhaus, I.; Rimbach, G.; Fink, C.; Roeder, T. Furbellow (Brown Algae) Extract Increases Lifespan in Drosophila by Interfering with TOR-Signaling. Nutrients 2020, $12,1172$. [CrossRef]

268. Huebbe, P.; Nikolai, S.; Schloesser, A.; Herebian, D.; Campbell, G.; Glüer, C.-C.; Zeyner, A.; Demetrowitsch, T.; Schwarz, K.; Metges, C.C.; et al. An extract from the Atlantic brown algae Saccorhiza polyschides counteracts diet-induced obesity in mice via a gut related multi-factorial mechanisms. Oncotarget 2017, 8, 73501-73515. [CrossRef] 